JENNY CRESCENCIA PAREDES AGUILAR

\title{
ABORDAGEM SEMÂNTICA APLICADA AO GERENCIAMENTO DE DADOS EM REDES DE SENSORES SEM FIO
}

\author{
Dissertação apresentada à Escola \\ Politécnica da Universidade de São \\ Paulo para obtenção do Titulo de \\ Mestre em Engenharia.
}

São Paulo 
Dedico este trabalho a meus pais José e Leonor por sempre ter acreditado, apoiado e conduzido sem medir esforços, por me dar amor, exemplo e cuidar de minha educação sempre. 


\section{Agradecimentos}

Aprendi, mais do que posso imaginar, sobre muitas coisas da vida ao realizar o mestrado. Essas coisas não cabem neste documento, mas nele estão registrados parte desse aprendizado e meus profundos agradecimentos:

Agradeço primeiro a Deus por sempre me acompanhar e me proteger, me dando forças nos momentos difíceis da vida e a meus pais José e Leonor por me permitir e me dar à oportunidade de demonstrar-Ihes que tudo é possível nesta vida através de esforço e dedicação, eles sempre me deram exemplo, ajuda e o apoio para que eu crescera como profissional e como ser humano.

Um agradecimento especial ao meu Orientador o Prof. Dr. Sergio Takeo Kofuji por toda a sua dedicação, apoio, entusiasmo, capacidade intelectual, disponibilidade e por confiar em mim. Foram fatores importantes para que este trabalho de mestrado se realizasse.

Agradeço também aos membros da banca de mestrado, pela paciência em ler meu trabalho e pelas suas valiosas sugestões e comentários. Agradeço ao grupo PAD, do LSI, da Escola Politécnica da USP por seu companheirismo e amizade. À equipe de HumanLab do Grupo PAD por seu apoio em tudo momento.

Um agradecimento os meus irmãos Duberly e Lizbeth que sempre estiveram comigo em todos meus passos neste projeto, por seu apoio espiritual e moral muito importante para meu desempenho. Agradeço a meu querido esposo Edwar por participar de mais uma jornada a meu lado, passamos por bons momentos e também por momentos difíceis durante o mestrado. Graças a ele, os momentos difíceis foram enfrentados com sabedoria e os bons momentos foram comemorados com muita alegria.

E um agradecimento especial a todas aquelas pessoas que deram seu apoio incondicional com seu tempo, seu conhecimento e sua experiência. 


\section{Resumo}

Nos últimos anos, o volume de dados e de informações disponíveis pelas RSSF cresceu consideravelmente por ser uma tecnologia que permite 0 desenvolvimento de aplicações em diversos tipos de ambientes (pervasivos, ubíquos, ambientes controlados e de difícil acesso), fazendo com que a preocupação em gerenciar esse conteúdo se tornasse maior. Dentre as propostas apresentadas, um grande número utiliza ontologias como forma de organizar e categorizar dados; existem duas questões chaves na gerência de dados nas redes de sensores sem fio: o armazenamento de dados e o processamento da consulta. $O$ presente trabalho propõe uma abordagem semântica para 0 gerenciamento dos dados dinâmicos e estáticos da rede, oferecendo um serviço de metadados sobre as RSSF e suas aplicações; esta técnica baseia-se na construção de ontologias que permitem escalabilidade, incremento de eficiência das atividades do ambiente e reusabilidade. Verificou-se mediante testes realizados baseados em consultas sobre a ontologia que o modelo proposto é apropriado, e que a busca baseada em semântica resulta na obtenção de dados das RSSF de forma mais adequada, quanto comparada com as abordagens tradicionais.

Palavras chaves: redes de sensores sem fio, semântica, ontologias, conhecimento, computação pervasiva. 


\begin{abstract}
In the recent years, the volume of data available from wireless sensor networks (WSN) has increased considerably. WSN is a technology that allows the development of applications in various types of environments (pervasives, Ubiquitous, controlled and with difficult access environment), doing that concern for content managing is greater. Among the proposals, many use ontologies as a way to organize and categorize data; there are two key issues in data management in WSN: data storage and query processing. The present work proposes a semantic approach to the management of dynamic and static data from the network, providing a metadata service on WSN and its applications; this technique is based on the construction of ontologies that allows scalability, enhancement of efficiency in the activities of the environment and reusability. It was verified by means of tests based on querys on the ontology that the proposed model is appropriate, and that the search based on semantics provided retrieval of data from WSN more adequately, as compared with traditional approaches.
\end{abstract}

keywords: Wireless sensor Networks, Semantics, Ontology, knowledge, Pervasive Computing. 


\section{Lista de Figuras}

Figura 1 Rede de Sensores Sem FIO (FLORIDO, 2008) 10

Figura 2 PILha de Protocolos das RSSF(F. C. DELICATO, 2005) 14

FIGURA 3 TAXONOMIA DE SOLUÇÕES DE ARMAZENAMENTO DE DADOS (DEEPAK ET AL., 2005) 18

FIGURA 4 A SEMÂNTICA EXISTE A DIFERENTES NÍVEIS DAS RSSF (LI ET AL., 2005) 23

FIGURA 5 GERENCIAMENTO DE DADOS NO WORLDWIDE SENSOR WEB (BALAZINSKA ET AL., 2007) 44

FIGURA 6 REGISTRO SIMPLES DA ONTOLOGIA ES3N (LEWIS ET AL., 2006) 47 FIGURA 7 TAXONOMIA INICIAL DA ONTOLOGIA PARA DADOS DO SENSOR (EID; LISCANO E SADDIK, 2007) 49

FIGURA 8 ONTOSENSOR EXTENSÃO DE IEEE SUMO (RUSSOMANNO; KOTHARI; THOMAS, 2005) 51

FIGURA 9 MSR SENSE (CHEONG ET AL., 2005) 52

FIGURA 10 CASOS DE USO PARA O WSN ONTOLOGY DEVELOPER 58

FIGURA 11 INTERFACE GRÁFICA DA FERRAMENTA PROTÉGÉ-OWL 58

FIGURA 12 CASOS DE USO PARA O WSN ONTOLOGY USER 59

FIGURA 13 CONCEPÇÃO DA ONTOLOGIA PARA RSSF 60

FIGURA 14 ONTOLOGIA GERAL 61

FIGURA 15 SUB-CLASSES DA ONTOLOGIA GERAL 62

FIGURA 16 ONTOLOGIA DA PLATAFORMA DO SENSOR 63

FIGURA 17 CLASSE GERAL DA PLATAFORMA DO SENSOR 65

FIGURA 18 CLASSE WSNMIDDLEWARE - ONTOLOGIA PLATFORM 66

FIGURA 19 CLASSE HARDWARE RESOURCE - ONTOLOGIA PLATFORM 67

FIGURA 20 RESTRIÇÕES DAS PROPRIEDADES DA CLASSE IDSENSOR 68

FIGURA 21 Classe da Plataforma da REDE (NETWORKPLATFORM) 69

FIGURA 22 ONTOLOGIA DO AMBIENTE

FIGURA 23 ONTOLOGIA DE SERVIÇO

Figura 24 Visualização do ambiente de uma Rede de Sensores Sem Fio (FLORIDO, 2008) 74

FIGURA 25 TELA DO CONSTRUTOR DE QUERIES TINYDBMAIN (MADDEN ET AL., 2005)77

FIGURA 26 RESULTADO DA CONSULTA DO TESTE - 1

FIGURA 27 ONTOLOGIA DE SERVIÇO 
FIGURA 28 ONTOLOGIA INFERIDA

FIGURA 29 JANELA DO DADO INFERIDO DO TESTE 1 UTILIZANDO PELLET 80

FIGURA 30 DADOS DOS SENSORES 81

Figura 31 Busca SemÂntica: Plataforma:=TMOteSky. FreqüÊNCIA:= $2.4 \mathrm{GHZ}$, E RSSI $<=-50$

FIGURA 32 BUSCA SEMÂNTICA: S.O.=TINYOS 1.X 83

FIGURA 33 RESULTADOS DA BUSCA DIRETA.

FIGURA 34 RESULTADOS DA BUSCA SEMÂNTICA. 86

FIGURA 35 RESULTADOS DAS BUSCAS DiRETAS VS. SEMÂNTICA. 87

FIGURA 36 ARQUITETURA DE CINCO CAMAdAS DA ABORDAGEM SEMÂNTICA PARA RSSF

99

FIGURA 37 ESQUEMA DO TRATAMENTO DE DADOS USANDO MSRSENSE (WOO ET AL., 2006) 101

FIGURA 38 BiblioteCAS NECESSÁRIAS PARA A IMPLEMENTAÇÃO DE ESTUdO dE CASO 103

Figura 39 Janela Para gerar JaVa SCHEMa Class - UtILIZANDO o Protégé 103 FIGURA 40 JANELA DE ECLIPSE 104

FIGURA 41 VISTA FRONTAL DO TMOTESKY (MOTEIV, 2007) 107

FIGURA 42 VISTA DA FACE INFERIOR DO TMOTESKY (MOTEIV, 2007) 108

FIGURA 43 APLICATIVO LISTEN 110 


\section{Lista de Quadros}

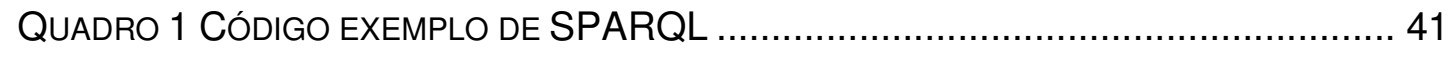

QUADRO 2 CÓDIGO SPARQL PARA LOCALIZAÇÃO E LEITURAS DOS NÓS ...................... 78

QUADRO 3 PARTE DE CÓDIGO EM JAVA PARA CARREGAR UMA ONTOLOGIA................. 105

QUADRO 4 PARTE DO CÓDIGO PARA REALIZAR INFERÊNCIA ................................. 106

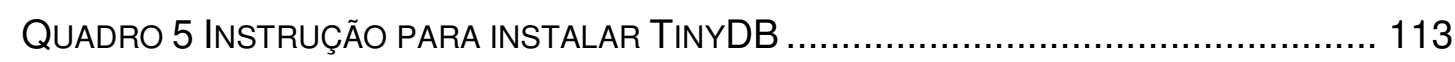

QUADRO 6 INSTRUÇÃO PARA A INSTALAÇÃO DO TINYDBMAIN................................ 113

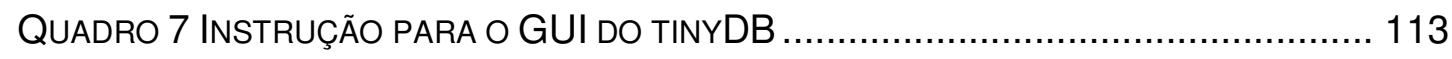




\section{Lista de Tabelas}

Tabela 1 Busca Direta: Plataforma:=TMOteSky. FreqüÊncia:= 2.4 GHZ, e RSSI

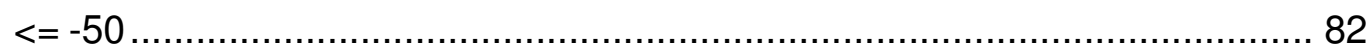

TABELA 2 BUSCA DIRETA: S.O. = TINYOS 1.X ............................................. 83

TABELA 3 DifERENTES FERRAMENTAS PARA O GERENCIAMENTO DE DADOS ................ 84

TABELA 4 Plataformas tíPICAS E COMPONENTES dE HARDWARE DAS RSSF .......... 108 


\section{Lista de Abreviaturas}

\begin{tabular}{|c|c|}
\hline ADC & Analog to Digital Converter \\
\hline API & Application Programming Interface \\
\hline $\mathrm{Cl}$ & Computação Inteligente \\
\hline DAC & Digital to Analog Converter \\
\hline DAML & DARPA Agent Markup Language \\
\hline DAWG & Data Access Working Group \\
\hline DBMS & Data Base Management System \\
\hline DIG & Description Interface Logics \\
\hline DL & Description Logics \\
\hline DMA & Direct Memory Access \\
\hline DMS & Data Management Systems \\
\hline DQL & DAML Query Language \\
\hline GUI & Graphical User Interface \\
\hline IA & Inteligência Artificial \\
\hline IEEE & Institute of Electrical and Electronics Engineers \\
\hline ISO & International Standards Organization \\
\hline JADE & Java Agent Development framework \\
\hline KIF & Knowledge Interchange Format \\
\hline LQI & Link Quality Indication \\
\hline MEMS & Micro Electro-Mechanical Systems \\
\hline MSR Sense & MSR Networked Embedded Sensing Toolkit \\
\hline OCML & Operational Conceptual Modeling Language \\
\hline
\end{tabular}




\begin{tabular}{|c|c|}
\hline OGC & Open Geospatial Consortium \\
\hline OIL & Ontology Inference Layer \\
\hline OWL-DL & OWL Description Language \\
\hline OWL-QL & OWL Query Language \\
\hline OWL & Web Ontology Language \\
\hline PA & Ponto de Acesso \\
\hline QoS & Qualidade de Serviço \\
\hline RDF & Resource Definition Framework \\
\hline RDF-S & Resource Description Framework-Schema \\
\hline RSSF & Rede de Sensores Sem fio \\
\hline RSSI & Received Signal Strength Indication \\
\hline SGD & Sistema de Gerência dos Dados \\
\hline SMP & Sensor Management Protocol \\
\hline SPARQL & Protocol and RDF Query Language \\
\hline so & Sistema Operacional \\
\hline SQL & Structured Query Language \\
\hline SQDDP & Sensor Query and Data Dissemination Protocol \\
\hline SSN & Semantic Sensor Networks \\
\hline TADAP & Task Assignment and Data Advertisement Protocol \\
\hline $\mathrm{TI}$ & Tecnologias de Informação \\
\hline UML & The Unified Modelling Language \\
\hline URI & Universal Resource Identifier \\
\hline XML & eXtensible Markup Language \\
\hline W3C & World Wide Web Consortium \\
\hline
\end{tabular}




\section{Sumário}

AGRADECIMENTOS .................................................................................... III

RESUMO......................................................................................................... IV

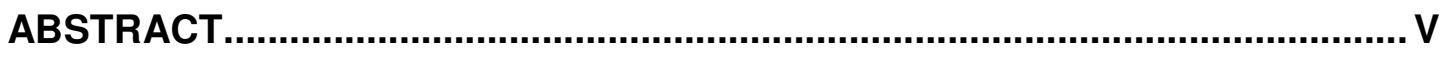

LISTA DE FIGURAS ................................................................................ VI

LISTA DE QUADROS.............................................................................. VIII

LISTA DE TABELAS ................................................................................... IX

LISTA DE ABREVIATURAS..........................................................................X

SUMÁRIO ..................................................................................................... XII

1. INTRODUÇÃO ........................................................................................ 1

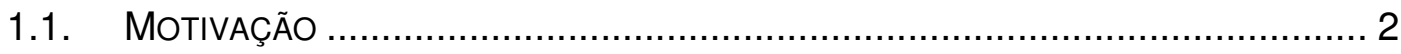

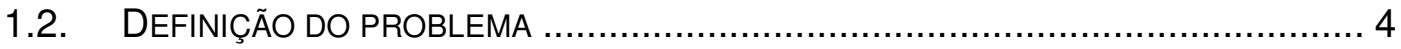

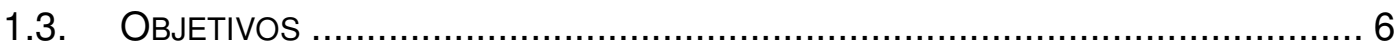

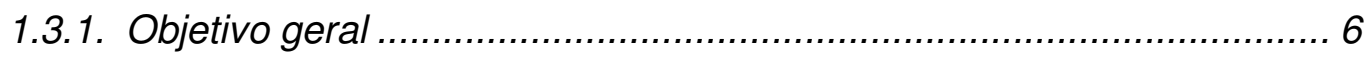

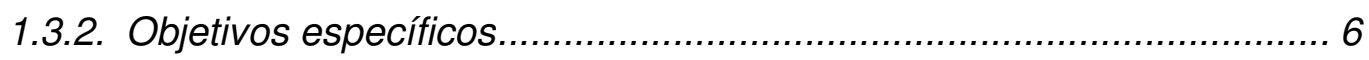

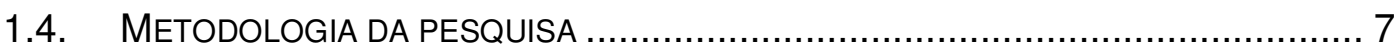

1.4.1. Conceituação do Projeto .............................................................. 7

1.4.2. Projeto Funcional................................................................ 8

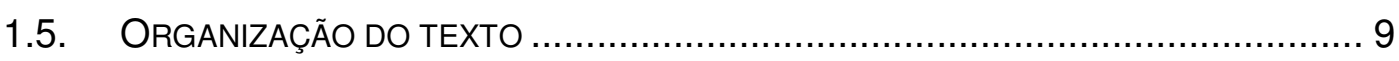

2. GERENCIAMENTO DE DADOS NAS REDES DE SENSORES SEM FIO .. 10

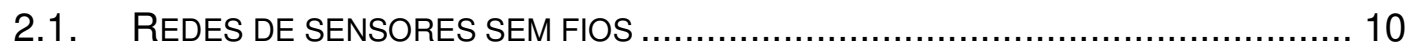

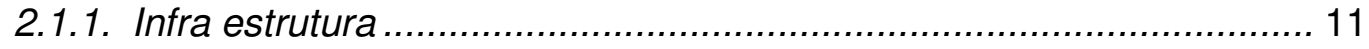

2.1.2. Pilha de protocolos .............................................................. 13

2.1.3. Modelos de Comunicações e entrega de dados em RSSF ............... 15

2.2. Gerenciamento de dados nas Redes de Sensores Sem Fio ................. 16

2.2.1. Armazenamento dos dados nas RSSF ................................... 17

2.2.2. Consultas e armazenamento centralizado ................................... 19

2.2.3. Armazenamento local e consulta geográfica................................. 19 
2.2.4. Armazenamento local com indexação distribuída 20

2.3. Protocolos de GeRENCIAMENTO de DADOS ....................................... 20

3. SEMÂNTICA NAS REDES DE SENSORES SEM FIO................................. 22

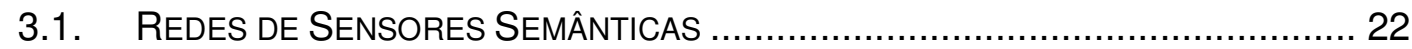

3.1.1. Ontologia para Redes de Sensores Sem fio ................................... 24

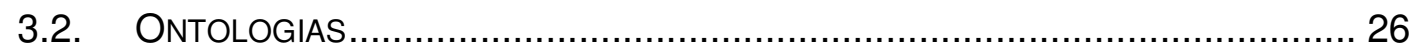

3.2.1. Linguagens de modelagem semântica ........................................... 27

3.2.2. A linguagem OWL de modelagem semântica ................................. 30

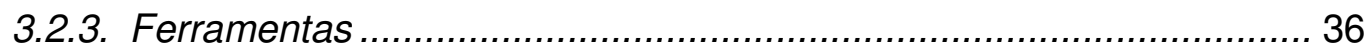

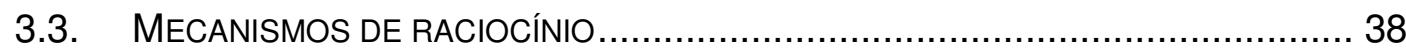

3.4. MECANISMOS DE RECUPERAÇÃO DE INFORMAÇÕES.................................. 40

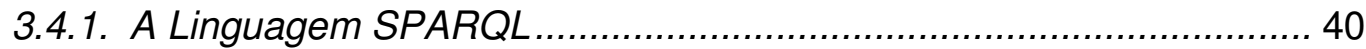

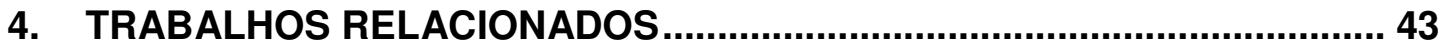

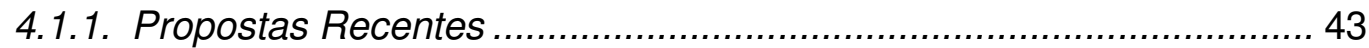

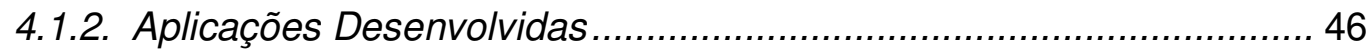

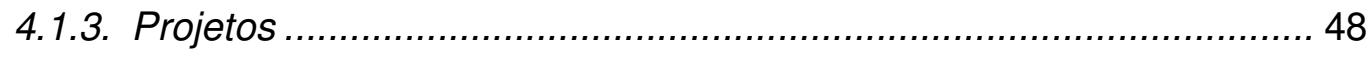

5. ONTOLOGIAS PARA O GERENCIAMENTO DE DADOS...........................53

5.1. DESENVOLVIMENTO DE ONTOLOGIAS PARA RSSF .................................... 53

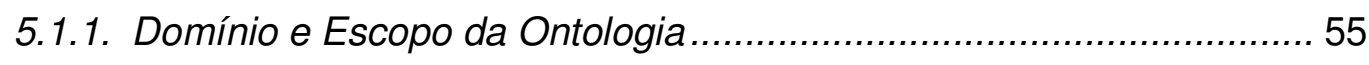

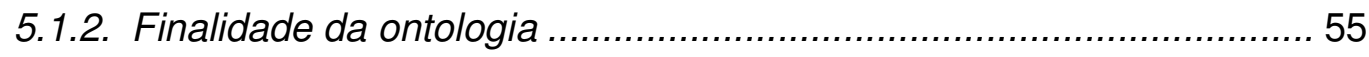

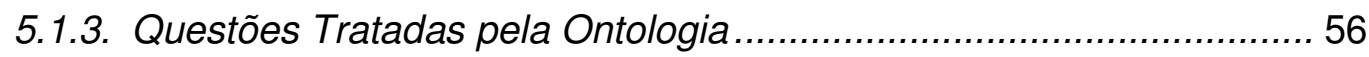

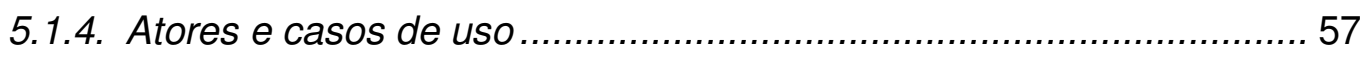

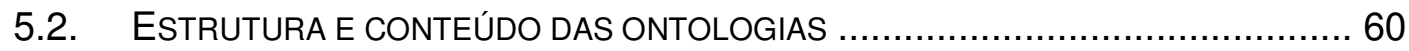

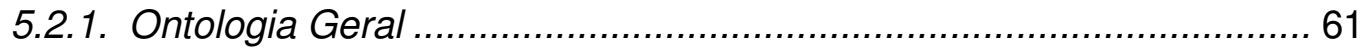

5.2.2. Ontologia da Plataforma (Platform)-classe do nó sensor

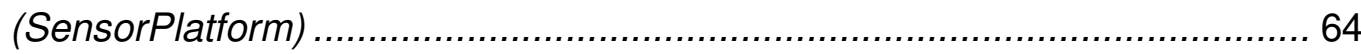

5.2.3. Ontologia da Plataforma (Platform)- classe da rede (NetworkPlatform) 68

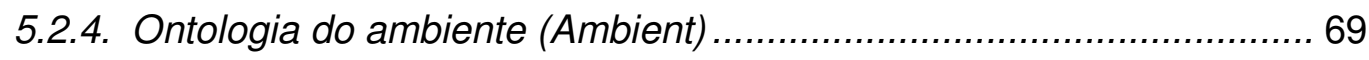

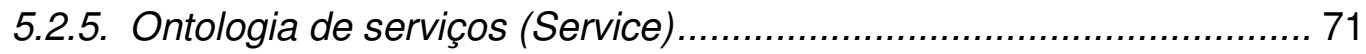

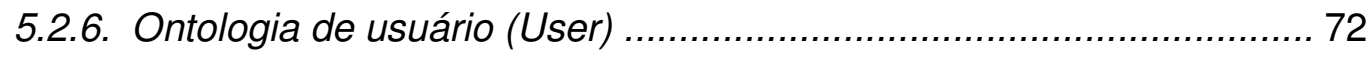


6. ESTUDO DE CASO

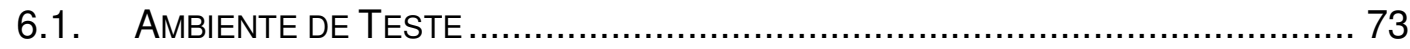

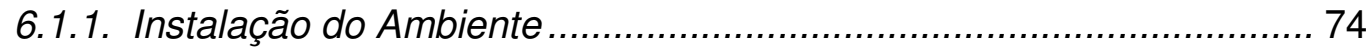

6.1.1.1. Ferramentas de apoio …………………............................. 75

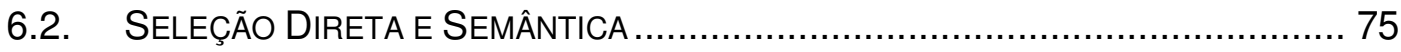

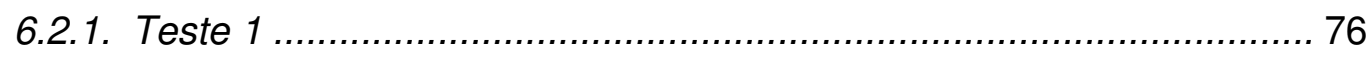

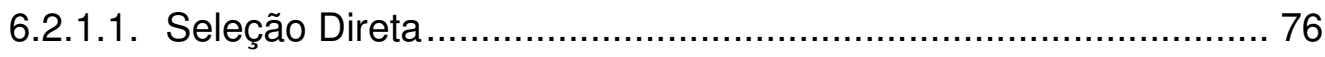

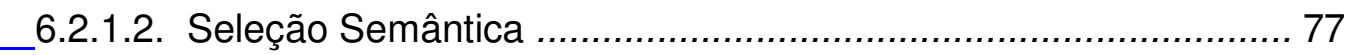

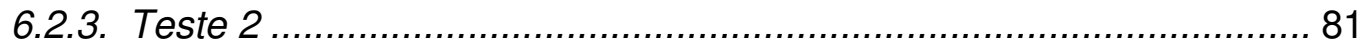

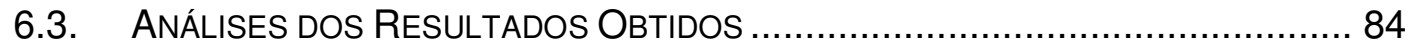

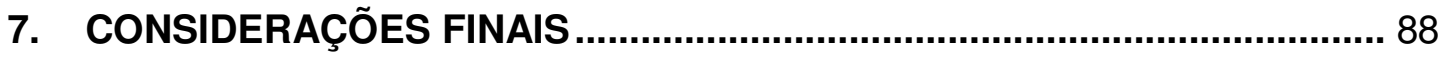

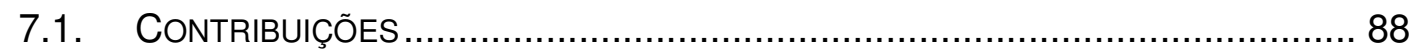

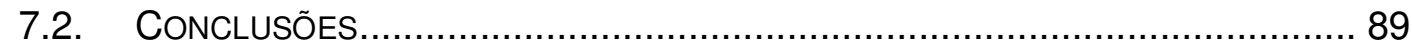

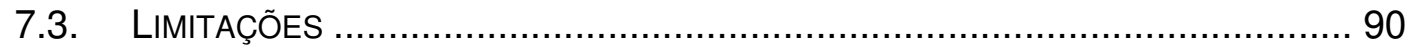

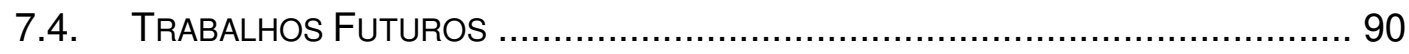

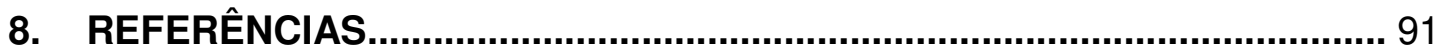

ANEXOS

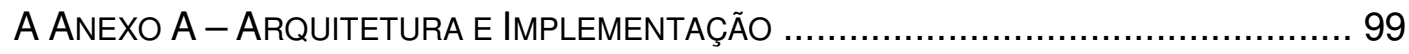

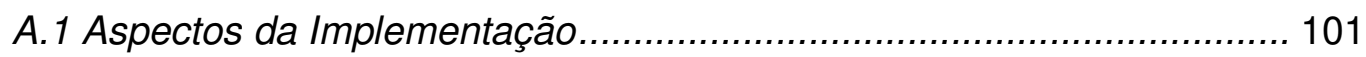

A.1.1 Implementação em Java .......................................................... 102

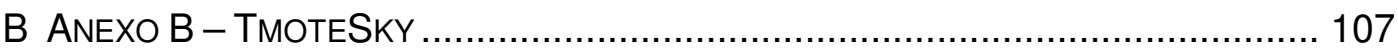

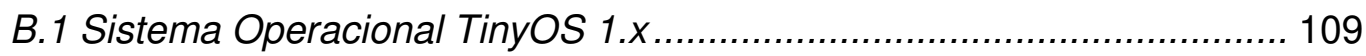

B.2 Aplicativos para visualização dos dados........................................... 110

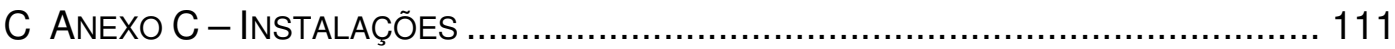

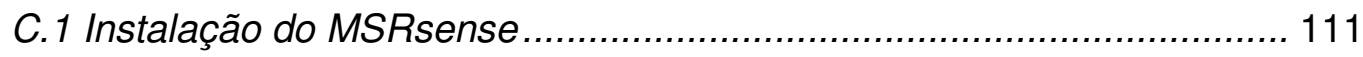

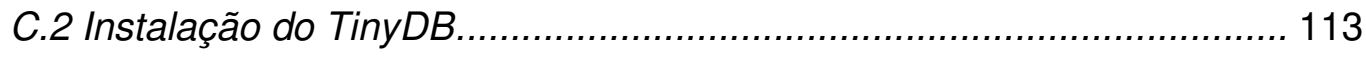




\section{Capítulo 1}

\section{Introdução}

As redes de sensores sem fio (RSSF) são redes $a d h^{1} c^{1}$ (topologia dinâmica e aleatória) (BASAGNI; CONTI; STOJMENIVIC, 2004), compostas por dispositivos de tamanho e custo limitado. Essas limitações restringem a capacidade do hardware e da reserva de energia desses elementos (SILVA, 2006).

Estes dispositivos são capazes de realizar sensoriamento, processamento e transmissão de informação através de enlaces (comunicação) sem fio (PEREIRA; AMORIM; STELLING, 2004). Os sensores atuam de forma colaborativa extraindo os dados e transmitindo-os para um ou mais pontos de saída de rede, chamados sorvedouros, para serem analisados e processados (ARAÚJJ; ANDERSON; SANTOS, 2007). É uma tecnologia promissora que constrói uma ponte entre o mundo físico e o mundo digital, permitindo extrair a informação alvo dos ambientes físicos, e conseqüentemente melhorar o monitoramento e controle dos ambientes.

Nos últimos anos, o volume de dados e de informações disponibilizadas pelas RSSF tem se tornado cada vez mais importante, visto que é uma tecnologia que vem sendo empregada em distintos ambientes (pervasivos, ubíquos, ambientes controlados, e de difícil acesso), dando origem a novos desafios no tratamento da informação e no gerenciamento de dados nas RSSF.

Devido à complexidade e volume de informação captada e tratada pelas redes de sensores é muito importante o gerenciamento de dados. Segundo Lewis et al. (2006) existem duas questões chaves no gerenciamento de dados nas redes de sensores: armazenamento de dados (como armazenar eficientemente dados) e processamento de consulta (como conseguir a recuperação de informação rápida e exata). Portanto, o gerenciamento de dados busca suprir a referida deficiência,

1 Rede $A D H O C$ é uma rede criada ou usada para um problema específico. Geralmente, numa rede ad hoc não há topologia predeterminada, nem controle centralizado e não requer uma infra-estrutura backbone ou pontos de acesso configurados antecipadamente. 
organizando os dados de forma a estruturá-los, armazená-los e agir adequadamente. No gerenciamento de dados, a aquisição e a fusão dos mesmos têm sido apontadas como uma opção que permite aperfeiçoar a operação das RSSF (WUN; PETROVI; JACOBSEN, 2007). Além disso, os esquemas distribuídos de gerência de dados devem ser processados quando os dados detectados são coletados das diferentes fontes em diferentes taxas (TSENG; WEN-CHIH; LEUNG, 2007).

Dentre as propostas apresentadas, um grande número utiliza semântica como forma de organizar e categorizar dados; esta tecnologia tem dois papéis fundamentais: os recursos são definidos usando descrições semânticas baseadas em ontologias ${ }^{2}$ e serviços semânticos; ontologias para o raciocínio permitem que a informação nova seja abstraída da informação coletada, buscando subsídios para se obter decisões mais precisas, aproveitando ao máximo o conhecimento disponível.

Nesta dissertação apresenta-se uma abordagem semântica para um Sistema de Gerenciamento de Dados (SGD), que fornece um serviço ciente de contexto dentro de um ambiente usando RSSF.

\subsection{Motivação}

As RSSF têm sido viabilizadas pela rápida convergência de três tecnologias: microprocessadores, comunicação sem fio e micro-sistemas eletro-mecânico (MEMS) (LOUREIRO et al., 2002). Uma RSSF pode ser usada para monitorar e controlar um ambiente; este tipo de rede é formado geralmente por centenas ou milhares de dispositivos autônomos que tendem a ser projetados em dispositivos de pequenas dimensões $\left(\mathrm{cm}^{3}\right.$ ou $\mathrm{mm}^{3}$ ) chamados nós sensores (LOUREIRO et al., 2003). Existem casos em que uma RSSF também pode ser composta por dispositivos chamados atuadores que permitem ao sistema controlar parâmetros do ambiente monitorado.

\footnotetext{
${ }^{2}$ Ontologia é uma especificação explícita de uma conceitualização, este conceito será detalhado no
} capítulo 3. 
Esse tipo de rede é aplicável em larga escala e em ambientes remotos ou de difícil acesso, utilizando um ou mais tipos de nós sensores. As RSSF podem ser homogêneas ou heterogêneas em relação aos tipos, dimensões e funcionalidades dos nós sensores (LOUREIRO et al., 2003); têm o potencial de serem empregados em outras áreas como monitoramento e controle remoto, smart house, automação, segurança, medicina/biologia, agricultura, entre outras. As RSSF podem mesmo mudar a maneira como os seres humanos interagem com os objetos. Entretanto, antes que estes objetivos possam ser conseguidos, há muitos desafios que devem ser superados.

As técnicas clássicas de recuperação de informação nas RSSF mostram um desempenho pobre na recuperação dos dados (EID; LISCANO; SADDIK, 2007). Além disso, estes dados precisam ser correlacionados e apresentados de uma maneira ciente ao contexto para resolver necessidades em tempo real e de uma maneira amigável para o usuário (HERBERT; O’DONOGHUE, 2006a).

Devido ao fato de que as RSSF permitem o desenvolvimento de aplicações de sensoriamento distribuído em cenários pervasivos, a infra-estrutura do gerenciamento de dados dentro de um ambiente pervasivo melhora o potencial para prever ou diagnosticar qualquer evento que pode acontecer no ambiente (ZUÑIGA; ROCA; KOFUJI, 2007).

Para gerenciar e controlar os dados poder-se-ia utilizar vários métodos relacionados a banco de dados. Uma geração nova de Database Management System (DBMS) requer sustentações semânticas adicionais para um gerenciamento de dados flexível e eficiente; isto inclui facilidades para a integração dos dados e otimização das consultas (NECIB; FREYTAG, 2003).

Considerando todos estes fatores é interessante a utilização da semântica baseada em ontologias construída com base nos padrões de World Wide Web Consortium (W3C) tais como eXtensible Markup Language (XML), Resource Definition Framework (RDF) e Web Ontology Language (OWL); estes fornecem uma plataforma para a interoperabilidade e uma semântica formal para o raciocínio, utilizando ferramentas para o tratamento, modelagem e consultas dos dados. 
Portanto, a motivação do presente trabalho é gerir o conhecimento implícito da RSSF e propiciar a utilização mais eficiente do crescente volume de dados na rede, fazendo uso das ontologias para o modelamento do contexto dos dados e decidindo quais inferências computacionais serão realizadas. Outra motivação da pesquisa é que a maioria dos autores está hoje concentrada em estudos sobre a eficiência energética, protocolos de roteamento, localização, etc. Poucos são os trabalhos apresentados até agora, sobre uma análise do gerenciamento de dados para redes de sensores sem fio, esta área de investigação está apenas começando (ZHI-FENG et al., 2006).

\subsection{Definição do problema}

As RSSF são capazes de coletar diversas características de um ambiente (temperatura, pressão, luminosidade, níveis de ruído, presença ou ausência de objetos, posição, velocidade, aceleração, etc.). Segundo Christopher e Campbell (2003) as redes de sensores tem um papel cada vez mais importante na mediação entre os mundos físicos e virtuais na Computação Pervasiva já que captam todo tipo de sinais físicos e os convertem em sinais elétricos para que possam ser processados.

Desta forma, se um sistema de gerenciamento de dados não estiver adequado para gerenciar o aumento da complexidade e o do conhecimento em um ambiente determinado, enfrentar-se-ia grandes dificuldades para obter êxito. $O$ gerenciamento de dados refere-se à capacidade de organizar os dados (como armazenar, tratar, visualizar e consultar os dados). A necessidade de uma abordagem apropriada dirigida a este problema é devido:

- À necessidade de gerenciamento de dados altamente relacionados e incompletos, para inferir informação e agir eficientemente, baseando-se no conhecimento gerado; nos ambientes pervasivos a relação dos dados permite a interoperabilidade com outros dispositivos. 
- À necessidade das associações entre os dados dos sensores são importantes para adicionar a capacidade de análises e apresentações das informações em tempo real, como também para o serviço de recuperação de informação rápida e eficiente limitando o uso de memória principal, para responder consultas complexas (LEWIS et al., 2006).

- A maioria das soluções atuais na área de RSSF fornece abstrações relativamente simples de consulta, e conseqüentemente não são apropriadas para as aplicações que requerem exigências mais sofisticadas de processamento de dados (HENRICKSEN; INDULSKA, 2006).

O software requerido para conectar os sensores e traçar as saídas do sensor à informação contextual útil é muito complexo, de modo que pesquisas recentes estão enfocadas em desenvolver um framework que assegure parte ou totalmente esta tarefa. Uma maneira eficiente de conseguir solucionar o problema de gerenciamento é fazer uso de descrições semânticas (HENRICKSEN; INDULSKA, 2006).

No transcurso deste trabalho, responderemos algumas questões, tais como:

- O gerenciamento de dados proposto através da semântica é a melhor opção?.

- Existem novas tecnologias de informação (TI) que trate o gerenciamento de dados das redes de sensores?.

- Quais são as novas tendências para gerenciar os dados nas RSSF?.

- Para que usar semântica nas RSSF?. 


\subsection{Objetivos}

\subsubsection{Objetivo geral}

O objetivo geral da dissertação é o desenvolvimento, a aplicação e a análise de um modelo semântico baseado em ontologias para a integração e gerenciamento eficiente dos dados nas RSSF.

\subsubsection{Objetivos específicos}

1) O primeiro objetivo específico é a análise do funcionamento dos dispositivos nós sensores TmoteSky (MOTEIV, 2007) para aquisição dos dados dos nós sensores (entradas, saídas, Analog to Digital Converter ADC, conectores externos, etc.);

2) o segundo objetivo é modelar a ontologia dos dados das RSSF usando a ferramenta Protégé para o controle dos dados;

3) o terceiro objetivo é definir as ontologias que associam a taxonomia da informação do nó sensor, a rede e o ambiente para consultar e interpretar os dados;

4) o quarto objetivo é determinar o ambiente para o estudo do caso no qual é utilizada a abordagem proposta;

5) finalmente, analisar os benefícios do gerenciamento de dados usando uma abordagem semântica e elaborar consultas utilizando as ferramentas propostas para comparar este modelo com outras abordagens e soluções. 


\subsection{Metodologia da pesquisa}

No decorrer do trabalho foi criada uma estrutura adequada que envolve todo o desenvolvimento da pesquisa. Esta estrutura foi dividida em etapas, enfocando cada parte do projeto separadamente conseguindo, assim, alcançar de forma seqüencial os objetivos específicos integrando-os no resultado final para alcançar o objetivo geral. A metodologia proposta especificamente para esta dissertação foi obtida como resultado da integração de várias metodologias e trabalhos relacionados (BALAZINSKA et al., 2007), (RUSSOMANNO; KOTHARI; THOMAS, 2005), ( O'DONOGHUE; HERBERT; 2006c).

\subsubsection{Conceituação do Projeto}

A metodologia adotada para realizar esta pesquisa iniciou-se com 0 levantamento bibliográfico baseado em livros, teses, artigos técnicos, publicações em congressos e revistas. A principal tarefa nesta fase é a elaboração do estado da arte através da pesquisa e revisão bibliográfica que incluem necessariamente dois temas centrais, redes de sensores sem fio, semântica e ontologias para RSSF.

Um fator importante que auxiliou na definição dos objetivos deste estudo foi identificar quais eram as preocupações dos projetistas em relação à pesquisa da abordagem em questão. Nesta fase de síntese escolheu-se a metodologia descrita em Noy e McGuinness (2001) disponibilizada no conjunto de ferramentas e documentação associadas ao Protégé-OWL.

A definição de uma estratégia adequada para a representação semântica de RSSF envolve fatores como a escolha de linguagens de modelagem, recuperação de informação, identificação de mecanismos e ferramentas de inferência. 


\subsubsection{Projeto Funcional}

Definida a organização da pesquisa no projeto de síntese e fundamento, esta etapa se encarrega de desenvolver, aplicar, analisar e avaliar cada uma das fases projetadas na etapa anterior. Esta etapa é composta por os seguintes passos:

- A primeira parte da pesquisa é a aquisição dos dados que são coletados mediante nós sensores Tmote-Sky e observação do formato do frame de dados;

- desenvolvimento das ontologias: o primeiro passo desta fase consiste na identificação de cenários genéricos em RSSF para adoção da abordagem semântica. Esta atividade também é conduzida, necessariamente, de modo iterativo e incremental. O seguinte passo desta fase é a elaboração de uma ontologia básica para representar os conceitos fundamentais relacionados às RSSF, seus componentes, recursos, dados, etc. A ontologia deve tornar explícito o conhecimento sobre o mecanismo de uma RSSF e definir uma taxonomia sobre conceitos comuns ao domínio de RSSF. Sua função mais relevante é prover uma base semântica extensível para o conjunto de dados em torno de recursos e aplicações visando promover a reutilização desses itens, a interoperabilidade entre diferentes aplicações e entre diferentes sistemas de middleware para RSSF;

- para o estudo do caso no Capítulo 6, foi aplicado o modelo semântico cujo cenário é um veículo onde será provado o sistema de consulta sobre a base de conhecimento;

- na obtenção dos resultados foram realizados testes (seção 5.2) para avaliar a utilização da inferência semântica e recuperação de informação.

A metodologia adotada na validação da pesquisa é dirigida à implementação. O modelo semântico é, basicamente, um conjunto de ontologias e um conjunto de mecanismos para explorar o uso dessa base de informações em cenários relevantes identificados ao longo do processo. Além disso, foi feita uma comparação com outras abordagens. 


\subsection{Organização do texto}

A seguir, apresenta-se a organização da dissertação:

- Capítulo 2: Este capítulo faz uma síntese dos conceitos na área em que o trabalho se insere, abordando temas como RSSF, características gerais, gerenciamento e armazenamento dos dados na RSSF;

- Capítulo 3: definem-se os conceitos da semântica e ontologias; descrevem-se linguagens de representação em ontologias com ênfase sobre a linguagem OWL; discutem-se os mecanismos e as políticas para exploração da base de conhecimentos baseados na inferência de informações; e, finalmente, apresentam-se estratégias de recuperação de informações em ontologias OWL. O Capítulo 2 e 3 são importantes para o entendimento dos capítulos seguintes, já que dão uma visão detalhada dos componentes estudados e conceitos gerais;

- Capítulo 4: Apresentam-se trabalhos relacionados considerados relevantes com ênfase na análise comparativa com a presente proposta;

- Capítulo 5: Este capítulo apresenta um conjunto de ontologias construídas para a modelagem dos dados das RSSF e descreve os mecanismos de raciocínio e estratégias de inferência usadas para aproveitamento eficiente da estrutura semântica no contexto das redes;

- Capítulo 6: Neste capítulo apresenta-se a validação da proposta e o caso do estudo onde são aplicadas as ontologias e seus resultados;

- Capítulo 7: Neste capítulo apresentam-se as considerações finais, contribuições, conclusões, trabalhos futuros e limitações;

Finalmente, temos os anexos, arquitetura e implementação para um caso de estudo, data chips dos Tmotes-Sky, instalação das ferramentas. 


\section{Capítulo 2}

\section{Gerenciamento de dados nas Redes de Sensores Sem Fio}

Nesta seção indicam-se as tecnologias selecionadas para o desenvolvimento da pesquisa e conceitos que envolvem o presente trabalho. Inclui-se uma breve resenha das RSSF, assim como conceitos de armazenamento, recuperação e processamento de dados. Finalmente, discutam-se algumas soluções e mecanismos relacionados ao gerenciamento de dados para serviços baseados em sensores de ambientes pervasivos.

\subsection{Redes de sensores sem fios}

Uma RSSF é um tipo de rede ad hoc, com uma série de características e requisitos específicos. Seu principal objetivo é realizar tarefas de sensoriamento de forma distribuída; portanto, essas redes funcionam como sistemas de aquisição de dados. Os dados são coletados através dos sensores que estão acoplados aos nós; esses por sua vez são distribuídos e entregues aos pontos de saídas da rede, chamados sorvedouros ou estação base. A Figura 1 mostra uma RSSF com uma estação base conectada a um computador.

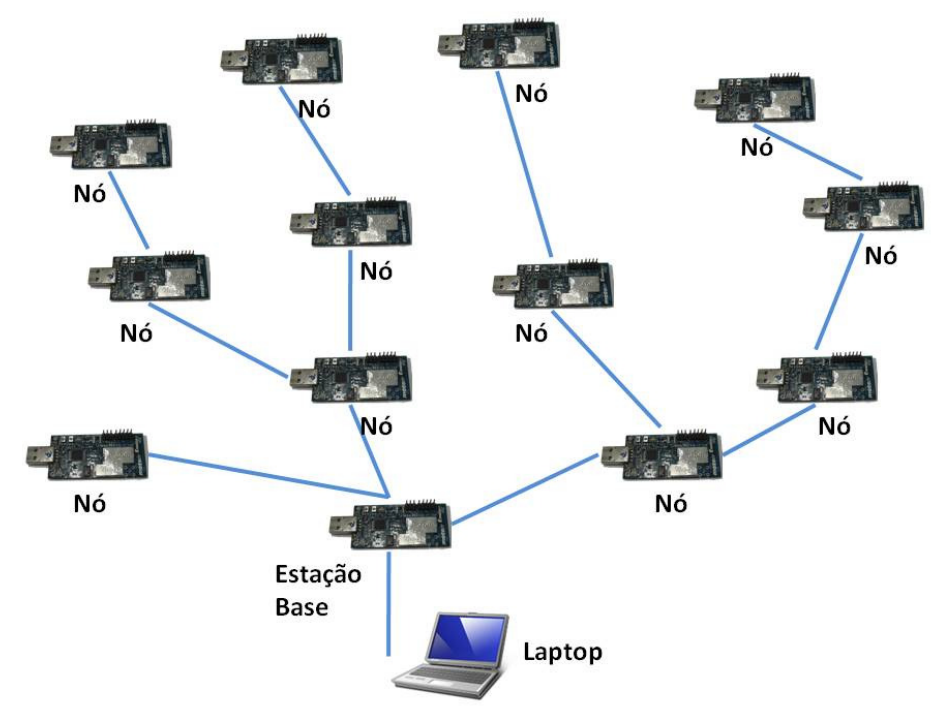

Figura 1 Rede de Sensores Sem Fio (FLORIDO, 2008) 
Quanto à sua estrutura, uma RSSF é organizada como um sistema com três componentes principais: infra-estrutura, pilha de protocolos; e aplicação (TILAK; ABU-GHAZALEH; HEINZELMAN, 2002). A infra-estrutura refere-se aos sensores físicos (suas características físicas e capacidades), ao número de sensores e à sua estratégia de instalação. A pilha de protocolos refere-se ao software que implementa as diversas camadas de protocolos existentes em cada nó da rede. O componente de aplicação representa os interesses ou consultas do usuário, que são traduzidos em tarefas de sensoriamento a serem executadas pela rede.

Uma RSSF é praticamente um tipo de sistema dependente da aplicação. Qualquer projeto ou solução proposta para esta rede deve levar em consideração os requisitos da aplicação a serem desenvolvidas, as características e restrições dos componentes dos nós, assim como as características do ambiente onde ficará a rede (RUIZ et al., 2004).

\subsubsection{Infra estrutura}

A infra-estrutura de uma RSSF consiste nos nós da rede e no seu estado de instalação no ambiente. Em geral, uma rede possui um ou mais nós de escoamento de dados, chamados de sorvedouros ou estações-bases, e diversos nós sensores. Estações bases fazem a interface entre a aplicação e a rede, servindo de ponto de entrada para a submissão dos interesses da aplicação e de concentrador dos dados enviados pelos nós sensores. Nós sensores contêm uma ou mais unidades de sensoriamento, e possuem capacidades de processamento e armazenamento limitadas. Sua função é coletar, eventualmente agregar, e transmitir seus próprios dados e os dos seus vizinhos para a estação base.

O hardware de um nó sensor típico é composto por quatro subsistemas principais: o de sensoriamento, o de processamento, o de comunicação e o de energia. Dependendo da aplicação, podem existir outros subsistemas adicionais, como por exemplo, o de localização (para determinar com precisão a posição de um nó) e o de movimento (para mover o nó para um local que permita a realização de uma tarefa) (RAGHUNATHAN; SPANOS; SRIVASTAVA, 2001). 
O subsistema de sensoriamento é geralmente composto pelos dispositivos de sensoriamento e pelo conversor de sinal analógico para digital (ADC). Uma RSSF pode conter muitos tipos de dispositivos de sensoriamento, como sísmicos, magnéticos, térmicos, visuais, infravermelhos, acústicos e radares. Tais dispositivos são capazes de monitorar uma ampla variedade de condições ambientais, incluindo: temperatura, umidade, movimento veicular, pressão, composição do solo, níveis de ruído, presença ou ausência de certos tipos de objetos, níveis de stress mecânico de objetos, e características como velocidade, direção e tamanho de um objeto. Os sinais analógicos produzidos pelos sensores a partir do fenômeno monitorado são convertidos em sinais digitais pelo ADC e são passados para o processador.

O subsistema de processamento que, em geral, está associado à unidade de armazenamento local, é responsável:

- Pela execução dos protocolos de comunicação e dos algoritmos de processamento de dados;

- pelo controle dos sensores e

- pela gerência dos procedimentos necessários para que os nós atuem de forma colaborativa.

O subsistema de comunicação pode ser composto por um dispositivo ótico ativo ou passivo, ou por um dispositivo de rádio freqüência (AKYILDIZ et al., 2002). A maioria dos projetos de RSSF atuais adota a comunicação via rádio freqüência.

A fonte de energia dos sensores consiste de uma bateria e um conversor CCCC. A bateria é um dispositivo complexo, cuja operação depende de diversas características como, por exemplo, suas dimensões, o material do qual é feita e a taxa de descarga (PINTO, 2004).

Além dos componentes de hardware descritos acima, o projeto de um nó sensor pode incluir um sistema operacional rudimentar. Quando presente, o sistema operacional deve gerenciar a operação do nó sensor da forma mais eficiente possível. Um exemplo de sistema operacional desenvolvido especialmente para 
sensores, e utilizado em grande parte do hardware hoje existente, é o TinyOS (HILL et al., 2000).

O estado de instalação da rede diz respeito à localização dos sensores no espaço físico, à densidade da rede e a possíveis deslocamentos no caso de sensores móveis. Centenas a milhares de nós sensores são instalados na área a ser monitorada, distanciados no máximo de algumas dezenas de metros uns dos outros. A densidade dos nós em uma RSSF pode ser tão alta quanto 20 nós $/ \mathrm{m}^{3}$ (SHIH et al., 2001).

Gerenciar um elevado número de nós com alta densidade requer uma criteriosa tarefa de manutenção de topologia por parte dos protocolos da rede. A alta probabilidade de falhas ou o possível deslocamento dos sensores dificultam essa tarefa (AKYILDIZ et al., 2002).

\subsubsection{Pilha de protocolos}

A pilha de protocolos usada pelos nós sorvedouros e sensores em uma RSSF é mostrada na Figura 2. Tal pilha consiste em cinco camadas horizontais e três planos verticais. As camadas são:

1. Camada de aplicação dependendo da tarefa de sensoriamento, diferentes tipos de software de aplicação podem ser construídos e usados para interagir com a RSSF. Segundo (AKYILDIZ et al., 2002) afirma que os protocolos para a camada de aplicação ainda são pouco explorados. Os autores sugerem três possíveis protocolos para apoio às aplicações:

a. Protocolo de gerenciamento de sensores (SMP); tem por finalidade tornar o hardware e o software das camadas mais baixas transparentes para as aplicações que gerenciam a rede de sensores.

b. Protocolo de anúncio de dados e designação de tarefas (TADAP); fornece, para as aplicações, interfaces para a emissão de suas consultas e para a coleta das respostas que chegam da rede. 
c. Protocolo de consulta e disseminação de dados (SQDDP) fornece à aplicação cliente da rede interfaces para conduzir a disseminação de seus interesses ou para receber anúncios de dados enviados pelos sensores.

2. Camada de transporte é a camada responsável por manter o fluxo de dados entre a origem e o destino, se a aplicação assim necessitar.

3. Camada da rede aborda detalhadamente a questão do roteamento em RSSF.

4. Camada de enlace de dados seu objetivo é assegurar conexões confiáveis em uma rede de comunicação.

5. Camada física abrange as técnicas de transmissão, recepção e modulação utilizadas na rede, as quais devem ser simples, porém robustas.

Os planos são: gerenciamento de energia, gerenciamento de tarefas e gerenciamento de mobilidade (AKYILDIZ et al., 2002).

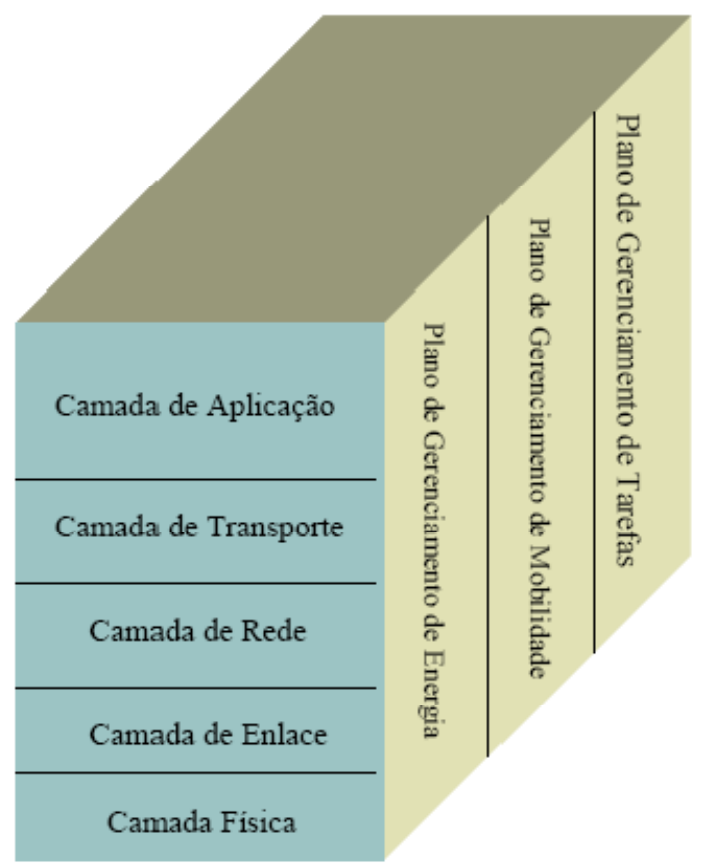

Figura 2 Pilha de Protocolos das RSSF (DELICATO, 2005) 
1. O plano de gerenciamento de energia; permite gerenciar a forma como os nós sensores utilizam sua energia. Por exemplo, um nó sensor pode desligar seu receptor após receber uma mensagem de um de seus vizinhos, evitando a recepção de mensagens duplicadas (AKYILDIZ et al., 2002).

2. O plano de gerenciamento de mobilidade permite detectar e registrar o movimento dos nós sensores, de modo a manter sempre uma rota para o usuário e a guardar, para cada nó, a informação sobre quem são seus nós vizinhos.

3. O plano de gerenciamento de tarefas; permite balancear e escalonar as tarefas de sensoriamento de uma região específica. Não é necessário que todos os sensores localizados na região alvo, definida pela aplicação estejam ativos ou contribuam ao mesmo tempo para a realização da tarefa.

Em síntese, os planos de gerenciamento são necessários para que os nós sensores possam trabalhar juntos de forma eficiente em termos de energia, rotear dados em redes com sensores móveis e compartilhar recursos entre si. Sem eles, cada sensor iria trabalhar apenas de forma individual.

\subsubsection{Modelos de Comunicações e entrega de dados em RSSF}

Geralmente, uma RSSF suporta dois tipos de comunicação (TILAK; ABUGHAZALEH; HEINZELMAN, 2002), comunicação de infra-estrutura e comunicação da aplicação.

A comunicação de infra-estrutura é responsável pela troca de mensagens entre os nós, necessária para configurar, manter e aperfeiçoar a operação da rede. Nesse tipo de comunicação estão incluídas, por exemplo, as trocas de mensagens para descobrir os caminhos dos sensores aos nós sorvedouros, e as mensagens usadas para a formação de clusters e para a eleição de líderes. 
A comunicação de aplicação é responsável pela transferência dos interesses da aplicação para a rede, a partir da qual a entrega de dados tem início, e pela transferência dos dados coletados sobre o fenômeno em estudo, desde sua origem até a aplicação destino. A adoção de um esquema de nomeação centrado em dados (HEIDERMANN, 2001) permite a descrição de interesses na forma de conjuntos de atributos de baixo nível, tais como o tipo do nó sensor, a área geográfica que se deseja monitorar, e o intervalo desejado para coletar os dados.

A partir do interesse definido pela aplicação, a rede começa a coletar e a enviar dados. O modelo de entrega de dados utilizado pela rede irá determinar o tipo de tráfego da aplicação. Em (TILAK; ABU-GHAZALEH; HEINZELMAN, 2002) são classificadas quanto ao modelo de entrega requerida pela aplicação, em quatro tipos: contínuos, dirigidas a eventos, iniciadas pelo observador e híbrido. No modelo contínuo, ou proativo, sensores comunicam seus dados continuamente a uma taxa pré-definida. No modelo dirigido a eventos, ou reativo, os sensores reportam informações somente se um evento de interesse ocorrer. Nesse caso, a aplicação está interessada apenas na ocorrência de um fenômeno específico. No modelo iniciado pelo observador ou (request-reply), os sensores reportam seus resultados em resposta a um pedido explícito (síncrono) da aplicação. A aplicação está interessada em obter uma visão instantânea (snapshot) do fenômeno monitorado. Finalmente, as três abordagens podem coexistir na mesma rede, gerando um modelo híbrido de entrega. O modelo da entrega de dados conduz a escolha do melhor tipo de protocolo de disseminação de dados a ser adotado na rede.

\subsection{Gerenciamento de dados nas Redes de Sensores Sem Fio}

A utilidade da informação recolhida não pode ser determinada a priori depende da aplicação (tracking, monitoramento, alerta). Existem dois casos extremos que podemos pensar para uma aplicação: uma delas que reporta o nível da temperatura de uma região remota e a outra que reporta um alarme de fogo; em ambos os casos, a temperatura é o parâmetro a ser medido. Mas, no primeiro caso o usuário final é interessado em coletar várias leituras do sensor por um largo período de tempo sem a necessidade de ter muita exatidão na informação coletada. No 
segundo caso precisa ser confiável e exata quando o fogo é detectado; todas estas características têm que ser consideradas para o gerenciamento de dados (BALDONI et al. 2006).

Segundo Lewis, et al. (2006) existem dois pontos chaves na gerência de dados nas redes de sensores:

- Armazenamento de dados (como armazenar eficientemente dados).

- Processamento da consulta (como conseguir a recuperação de informação rápida e exata).

A primeira abordagem é resolvida com alguma eficiência, armazenando dados de forma local, central ou distribuída. A segunda abordagem é fundamental em nossa pesquisa, no processamento da consulta o gerente dos dados é o encarregado de reduzir e resumir dados on-line, proporcionando simultaneamente armazenamento e adicionando facilidade para as análises off-line (GEHRKE; MADDEN, 2004) (consumo de poder mínimo). Também deve fornecer uma interface que permita que o usuário compreenda, colete, processe e controle o estado da rede e os dados, (tais como, médias, momentos, histogramas, sumários estatísticos) gerados em tempo real (GEHRKE; MADDEN, 2004).

\subsubsection{Armazenamento dos dados nas RSSF}

O armazenamento é extremamente limitado, desde que os dados gerados pelos sensores podem facilmente saturar a memória disponível, em geral os dados podem ser armazenados de forma local, distribuída ou centralizada (KEPHART; CHESS, 2003).

Muitos esquemas foram propostos para a gerência de dados em redes dos sensores. As idéias principais se resumem na comunicação requerida para o armazenamento de dados e comunicação requerida para a consulta. A Figura 3, descreve uma representação entre 0 armazenamento centralizado e 0 armazenamento local. 


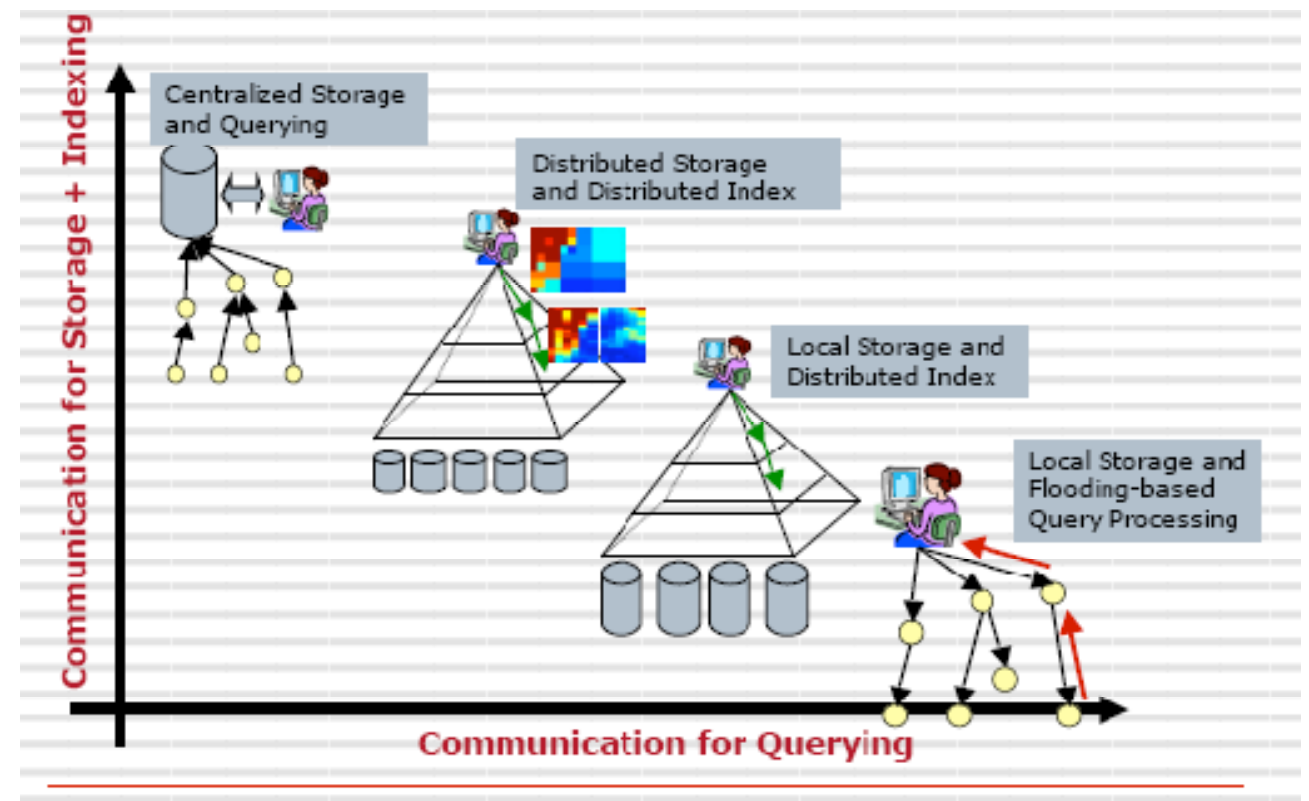

Figura 3 Taxonomia de Soluções de armazenamento de dados (DEEPAK et al., 2005)

Em um extremo, está uma aproximação convencional da gerência de dados centralizada, onde todos os dados úteis do sensor são transmitidos dos sensores a um repositório central com amplos recursos de poder e de armazenamento.

Para determinar que mecanismo de armazenamento e processamento de consulta é preciso de muitos parâmetros e depende da aplicação, como por exemplo:

- A distribuição dos nós é de curta ou longo prazo?.

- Qual é a quantidade de energia para armazenamento e processamento?.

- Qual é o tipo de consultas dos dados e sua freqüência?.

- Quantos dados úteis do sensor são gerados pela rede? (DEEPAK et al., 2005).

Existem diferentes soluções para tratar a grande quantidade de exigências nas RSSF, estas soluções são diferentes às de um sistema comum de armazenamento de redes distribuídas, devido a dois aspectos: 
1. A restrição de energia e de comunicação.

2. Os dados nas RSSF apresentam correlações espaço-tempo, para ser explorado no armazenamento, processamento e consulta dos dados.

\subsubsection{Consultas e armazenamento centralizado}

É o enfoque convencional para armazenar os dados dos nós sensores, todos os dados coletados são armazenados em um repositório central externo ao ambiente em observação como é mostrado na Figura 3. As consultas sobre estes dados não incorrem em nenhum custo adicional porque são residentes no armazenamento externo à rede. As vantagens são armazenamento centralizado persistente (como banco de dados) e a consulta rápida e eficiente.

As consultas sobre estes dados são feitas na posição central e não incorrem em nenhum custo de energia na rede do nó sensor. $\mathrm{O}$ armazenamento centralizado é apropriado para uma baixa taxa de dados.

\subsubsection{Armazenamento local e consulta geográfica}

Na Figura 3 mostra um esquema do armazenamento inteiramente local onde todos os dados úteis do sensor são armazenados localmente em cada nó, e as consultas se enviam às posições onde os dados são armazenados.

Desde que os dados são armazenados localmente nos nós que os produziram, a consulta pode incorrer em um custo de energia elevada, porque os dados podem residir em qualquer lugar na rede, uma consulta que não se limita explicitamente o espaço físico da consulta deve ser espalhada a todos os nós do armazenamento na rede.

Se somente algumas perguntas fossem emitidas durante a vida de uma rede, responder a estas perguntas pode envolver pouco custo de comunicação. Um 
grande número de perguntas envolve uma comunicação significativa, entretanto, podem acabar as reservas da energia de uma rede. Muitas das idéias iniciais estão dentro do contexto de Directed Diffusion (INTANAGONWIWAT; GOVINDAN; ESTRIN, 2000). As desvantagens deste tipo de armazenamento são que os dados serão perdidos se o nó sensor falha; cada sensor dever agir como um processador de consultas o qual incorre no alto overhead ${ }^{3}$.

\subsubsection{Armazenamento local com indexação distribuída}

Existe um crescente trabalho em esquemas da indexação dos dados para redes de sensores (RATNASAMY et al., 2002) (GREENSTEIN, 2003). Este mecanismo consiste em armazenar os dados a nível local em cada nó e construí estruturas de índices distribuídos para fazer consulta mais eficiente.

Estas técnicas diferem nos mecanismos da agregação de dados, são baseadas na idéia do hashing geográfico e de replicação estruturada. Para fazer consultas eficientes requer-se pouco overhead de comunicação. Sua desvantagem, assim como no método anterior,é que os dados são perdidos se o nó sensor falha.

\subsection{Protocolos de Gerenciamento de dados}

A importância colocada em técnicas de gerência dos dados aumentou devido aos dispositivos sensores de baixo custo e ao surgimento de ambientes pervasivos. Os ambientes pervasivos atuais e futuros podem produzir grandes volumes de dados dos sensores.

Todos os dados dentro de Data management system (DMS) requerem um processamento apropriado e devem ser analisados no contexto da informação disponível. É essencial um modelo semântico apropriado para o processamento e a análise dos dados. Refere-se a um modelo conciso que represente a informação no

3 Em ciência da computação overhead é geralmente considerado qualquer processamento ou armazenamento em excesso, seja de tempo de computação, de memória, de largura de banda ou qualquer outro recurso que seja requerido para ser utilizado ou gasto para executar uma determinada tarefa 
contexto do domínio da aplicação. A arquitetura do DMS permite cenários do mundo real a serem examinados e analisados.

Cada protocolo do DMS é projetado a executar determinadas regras de Qualidade de Serviço (QoS) . Existem protocolos do DMS em quatro áreas chaves definidas (O'DONOGHUE; HERBERT, 2006b):

- Perfis de usuário. - Os perfis dos usuários utilizam técnicas de filtragem dos dados para reduzir a sobrecarga da informação e retornar somente a informação relevante ao usuário. Aqui as variáveis cientes ao contexto no tempo real estão sendo analisadas baseadas nas propriedades do usuário, assim melhora-se o diagnóstico.

- Prioridade dos dados. - O protocolo da prioridade dos dados do DMS examina as leituras em tempo real. Então, de acordo com as variáveis do contexto a prioridade do evento mudará dinamicamente.

- Validação dos dados. - Falhas ou erros do sensor podem ocorrer devido ao mau funcionamento ou à interferência de dispositivos externos. Dentro de um ambiente no qual se está realizando testes em tempo real, tais falhas e erros são consideráveis. Um nível de tolerância a falhas é necessário, construído para validar os dados. Entretanto se, este nível de tolerância é excedido um alarme da falha ou do erro do sensor é provocado.

- Consistência dos dados. - Os dispositivos móveis múltiplos podem ser ativos dentro de um ambiente pervasivo. Simultaneamente, um ou mais membros podem necessitar o monitoramento e leitura em tempo real dos dados que estão sendo validados, precisa-se assegurar que todos os dispositivos móveis contêm a informação necessária. 


\section{Capítulo 3}

\section{Semântica nas Redes de Sensores Sem Fio}

Neste capítulo, descrevem-se a semântica nas redes de sensores e os formatos usados para construir uma ontologia de Web Semântica: XML, RDF e OWL; apresentam-se também algumas linguagens de consulta e ferramentas para interpretar e explorar ontologias nestes formatos.

\subsection{Redes de Sensores Semânticas}

A natureza data-centric das redes do sensor fornece uma abordagem inovadora para resolver uma grande quantidade de aplicações. Além disso, as RSSF sofrem alguns inconvenientes (NI et al., 2006).

- A maioria de soluções é baseada na disposição de sensores homogêneos;

- cada solução é geralmente para uma aplicação específica;

- a solução é geralmente uma abordagem de engenharia sem um framework comum;

- não existe nenhum padrão que permita uma comunicação entre diferentes sensores a diferentes níveis;

Segundo Ni et Al. (2006) "Semantic sensor network" (SSN) atenua os inconvenientes mencionados anteriormente. Uma SSN é uma rede de sensores heterogêneos que permite a colocação de etiquetas dinâmicas, da informação semântica aos dados capturados pelos sensores, para o monitoramento eficiente e sistemático de ambientes dinâmicos. As SSN têm as seguintes vantagens:

- A colocação de etiquetas da informação semântica aos dados sensoriais para a manipulação eficiente de dados sensoriais heterogêneos distribuídos em grande escala. 
- SSN pode fornecer uma base teórica à pesquisa das RSSF a diferentes níveis como se mostra na Figura 4.

- SSN pode ajudar a desenvolver uma estrutura baseada em semântica para resolver sistematicamente várias aplicações.

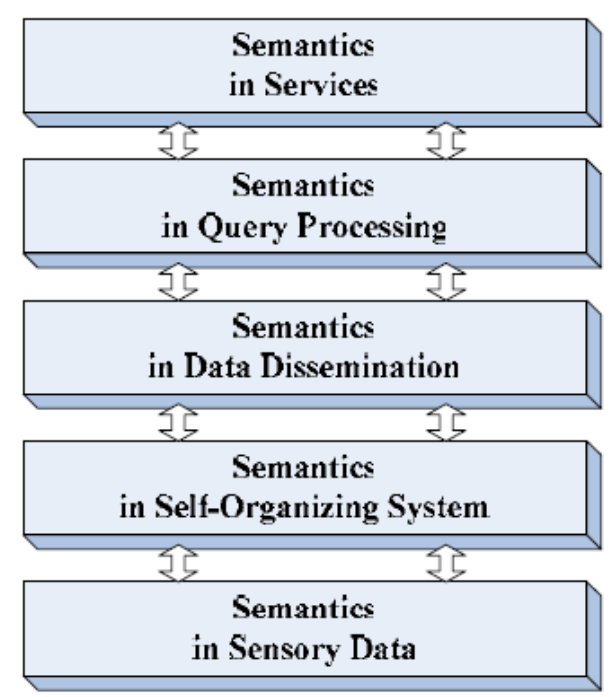

Figura 4 A semântica existe a diferentes níveis das RSSF (LI et al., 2005)

Segundo Xue, Pan e Li (2007) a semântica dos dados sensoriais é criada quando os dados são capturados do ambiente, é difícil de processar ou interpretar os dados capturados pelos sensores sem uma semântica explícita. Cada sensor gera um tipo de dado; para fazer estes dados significativos, nós necessitamos unir-Ihe semântica, isto é feito pelo anexo de um Tags semântico (XUE; PAN; LI, 2007).

O Tag semântico de dados capturados é a descrição necessária sobre o ambiente onde os dados de sensoriamento foram gerados e a descrição dos dados próprios do sensoriamento. O Tag semântico deve incluir:

\section{Metadados}

São as descrições necessárias sobre os dados capturados pelos sensores. Por exemplo, tomemos os dados capturados de um sensor de temperatura. A meta data 
pode incluir o tipo de medida neste caso é a temperatura, a unidade de medida que pode ser Fahrenheit ou Celsius e a precisão da medida. A meta data depende geralmente da capacidade dos dispositivos de sensoriamento, estes dispositivos podem ter diferentes tipos de meta dados.

\section{Informação Contextual}

Segundo (FERREIRA et al., 2005), "Contexto é qualquer informação que pode ser usada para caracterizar a situação de uma entidade que é considerada relevante entre o usuário e a aplicação". Considerando a forma como as condições do contexto são capturadas, classifica-se as fontes de contexto em:

- Percebida pelos Sensores: Informação gerada através dos sensores com alguma precisão.

- Definida pelo Usuário: O usuário fornece alguns valores à aplicação durante uma configuração. Valores por default devem sempre estar disponíveis.

- Fornecida por um Serviço: A informação é dada por algum fornecedor de serviços.

- Aprendida: A informação é gerada observando o comportamento do usuário.

- Inferida: A informação é gerada pelo processamento de outras informações de contexto em tempo de execução baseadas em regras definidas em tempo de desenho.

\subsubsection{Ontologia para Redes de Sensores Sem fio}

A modelagem dos dados adquiridos pelos sensores em um formato OWL, garante sua interoperabilidade com outros sistemas, como serviços web semânticos, entre outras por estar desenvolvida em uma sintaxe XML. Para gerar um grau maior de informação aos dados, são modelados também, o ambiente, os sensores e a rede. Para isso são definidos Ontologias, estes modelos de dados enriquecem as informações e facilitam a iteração do usuário com o ambiente (ZUÑIGA; ROCA; KOFUJI, 2007). 
O desenvolvimento das ontologias é importante para compartilhar a compreensão comum da estrutura da informação entre pessoas e agentes de software, permitindo reusar o domínio de informação, fazendo suposições explícitas do domínio e analisando o domínio de conhecimento (NOY; FERGERSON; MUSEN, 2000). Podem-se distinguir três níveis no contexto de RSSF: nível do dispositivo, nível do ambiente e nível da aplicação.

- O nível de dispositivo consulta todas aquelas falhas no dispositivo físico, a aplicação depende de dados, como a memória, bateria, poder de processamento etc.

- O nível do ambiente consulta tudo que é externo ao dispositivo físico, tal como, conectividade da rede, posição, nós vezinhos, etc.

- O nível da aplicação consulta toda a informação da aplicação especifica, tal como parâmetros de QoS, valores coletados do sensor, consultas e descrições da tarefa.

É importante indicar que o contexto da execução da RSSF é extremamente dinâmico, desde os estados da rede até a aplicação. Os nós da RSSF podem ter sua bateria esgotada e nós novos podem ser adicionados á rede após a distribuição inicial. É muito provável que a largura de faixa e a qualidade das ligações sem fio mude ao longo do trabalho da rede. Além disso, os interesses da aplicação podem ser verdadeiramente dinâmicas, com parâmetros de perguntas e exigências de QoS.

Segundo Delicato et Al. (2006) existe situações em que seria conveniente adicionar semântica para acessar e usar uma RSSF:

- Para localizar redes que, potencialmente, satisfaçam os interesses de uma aplicação, dada uma descrição em alto nível dos serviços requeridos. O objetivo neste caso é descobrir o endereço (URL) do sorvedouro ou ponto de acesso de tais RSSF, através do qual as aplicações podem acessar e usar os serviços das RSSF. Neste caso, o protocolo UDDI, usado para descobrir RSSF, deveria ser adicionado às capacidades semânticas. Enfrentar essa situação está fora do âmbito do nosso trabalho; 
- para determinar se a tarefa requerida pela aplicação pode ser totalmente realizada pela RSSF que foi escolhida, dada a descrição detalhada da tarefa, incluindo os requerimentos de QoS;

- uma vez iniciada a tarefa, compartilhar conhecimento do contexto de execução, permitindo:

a) Aos nós sensores, enviar informações sobre a rede e os estados atuais da aplicação;

b) as aplicações, monitorar tal estado;

c) aos componentes de serviço, verificar se um determinado estado de execução é válido.

\subsection{Ontologias}

Uma das definições mais citadas na literatura, Gruber (1993) define a ontologia como uma especificação explícita de uma conceitualização. Como esta definição é genérica e abstrata houve espaço para que fosse criada certa confusão em torno da definição de ontologia.

Uma especificação explícita de uma conceitualização pode abranger desde simples vocabulários até teorias axiomatizadas. Assim, (GUARINO, 1998) propõe uma gradação para as diversas interpretações dadas ao termo ontologia:

- Léxico: vocabulário com definições de linguagem natural;

- Taxonomia simples;

- Tesauro: taxonomia mais termos relacionados;

- Modelo Relacional: uso irrestrito de relações arbitrárias;

- Teoria totalmente axiomatizada. 
De acordo com Sowa (1999) o termo ontologia tem um sentido específico quando se trata de organização da informação, diferente do adotado na filosofia e em outras áreas. Ontologia é um catálogo de tipos de coisas em que se supõe existir um domínio, na perspectiva de uma pessoa que utiliza uma determinada linguagem.

Compartilhar, reutilizar conhecimento sobre um domínio, tornar explícitas proposições assumidas sobre um domínio, separar conhecimento sobre um domínio de conhecimento operacional, e analisar o conhecimento sobre um domínio são as razões mais comuns para o desenvolvimento de uma ontologia (DEVEDZI, 2002).

Trabalhos pioneiros para realização da Web semântica são voltados para o desenvolvimento de linguagens tais como Resource Description Language (RDF), DAML+ Ontology Inference Layer (OIL), DARPA (CONSORTIUM W.W.W.W., 2007) e Web Ontology Language (OWL).

\subsubsection{Linguagens de modelagem semântica}

Segundo (CORCHO; FERNANDEZ; PEREZ, 2003) no começo dos anos 1990 foram criadas linguagens para a implementação de ontologia baseadas em Intelligencia Artificial (IA). Basicamente o paradigma de Representação do Conhecimento lidava com linguagens de ontologia baseadas em lógica de primeira ordem (por exemplo, Knowledge Interchange Format ou KIF (GENESERETH, 2006), com frames combinados com lógica de primeira ordem (por exemplo, Ontolingua (ONTOLINGUA, 2005), ou com lógica descritiva como, por exemplo, LOOM (PROJECT LOOM, 2006).

O crescimento da Internet conduziu à criação das linguagens de ontologia que exploram características da Web. Tais linguagens são chamadas, geralmente, linguagens de ontologia baseadas em Web ou linguagens de marcação de ontologia (Ontology markup languages).

No ano de 2001, o W3C criou um grupo de trabalho chamado Web-Ontology (WebOnt), com o objetivo de desenvolver uma nova linguagem de marcação de 
ontologia para a Web Semântica, chamada Ontology Web Language ou OWL. Atualmente, as linguagens para a representação de ontologias são divididas em dois grupos: i. As baseadas na Lógica de Primeira Ordem; ii. As baseadas em XML. Estes grupos de linguagens possuem diferentes expressividades e propriedades computacionais e são descritas a seguir.

1. Linguagens Baseadas em Lógica de Primeira Ordem: são apenas citadas aqui, pois não fazem parte do foco do trabalho.

- Knowledge Interchange Format (KIF) (GENESERETH, 2006);

- Ontolingua (UNIVERSITY, 2006);

- Loom (PROJECT LOOM, 2006);

- Operational Conceptual Modeling Language (OCML) (K. M. INSTITUTE, 2006);

- Frame Logic (FLogic) (KIFER; LAUSEN; WU, 1990).

2. Linguagens Baseadas em Extensible Markup Language (XML): A XML provê uma sintaxe para documentos estruturados e permite a definição de tags específicas de uma área, mas não impõe restrições semânticas sobre o significado. Apesar de estas linguagens serem baseadas em $\mathrm{XML}$, elas obedecem a fundamentos de IA, por exemplo, lógica descritiva, redes semânticas, frames, dentre outros, permitindo que muitas ontologias estejam sendo desenvolvidas em laboratórios de IA (GÓMEZ; CORCHO, 2002).

- RDF: Segundo (LASSILA; SWICK, 1999) a linguagem RDF é voltada para a representação de metadados sobre recursos da Web, incluindo coisas que podem ser identificadas, ainda que não possam ser diretamente recuperadas. Os recursos são descritos em termos de propriedades e valores de propriedades como um grafo, cujos nós e arcos representam os recursos; suas propriedades e valores em declarações na forma de triplas contendo sujeito, predicado e objeto. 
A linguagem RDF provê um arcabouço comum para expressar informações que precisam ser processadas por aplicações, além de permitir compartilhar essas informações entre as aplicações sem perda de significado. A linguagem RDF é baseada no conceito de identificadores Web chamados identificadores uniformes de recursos (URI) (WALSH, 1998). Um identificador URI pode ser criado para referenciar desde qualquer coisa acessível pela rede, tais como documentos, imagens e serviços, até coisas não acessíveis pela rede, como empresas e pessoas, além de conceitos abstratos.

- DARPA Agent Markup Languaje (DAML) é uma linguagem que foi desenvolvida como uma extensão para XML e RDF. Provê uma infraestrutura básica que permite às máquinas fazerem a mesma classificação de inferências simples que os seres humanos fazem. Um sistema em DAML pode inferir conclusões que não estão explicitamente declaradas em DAML (BERNERS-LEE, 2006).

- Ontology Inference Layer (OIL) é uma camada de inferência e representação baseada na Web, que combina a utilização de modelagem de primitivas provenientes das linguagens baseadas em frames com a semântica formal. A sintaxe de OIL é baseada em RDF-Schema e provê definição de classes e slots (relações), e um conjunto limitado de axiomas (SU; ILEBREKKE, 2002).

- A linguagem DARPA Markup Language + Ontology Inference Layer (DAML+OIL) foi construída com base na linguagem DAML em um esforço para combinar vários componentes da linguagem OIL. É construída sobre padrões W3C tais como RDF e RDF-Schema, e estende estas linguagens com primitivas de modelagem mais ricas, além de possuir semântica clara e bem definida (CONNOLLY et al., 2001).

- A Ontology Web Language (OWL) é a linguagem utilizada no desenvolvimento do trabalho, será detalhada na seção seguinte. A OWL foi desenvolvida como uma extensão do vocabulário RDF e derivada da 
linguagem ontológica para Web DAML+OIL (DJURIC; GASEVIC; DEVEDZIC, 2005).

\subsubsection{A linguagem OWL de modelagem semântica}

A linguagem OWL oferece recursos adicionais para interpretação de conteúdos que não estão presentes em linguagens XML, RDF e RDF Schema (RDFS) (CONSORTIUM W.W.W.W., 2007), tais como relacionamento entre classes (e.g., disjunção), cardinalidade, igualdade, características de propriedade (e.g., transitividade), e classes enumeradas, também conhecidas como nominais. Na OWL é possível também estabelecer nas sub-classes restrições de propriedades herdadas das super-classes, podendo ter tais restrições relações com outras classes.

Esses recursos facilitam o processamento e integração automáticos de informação disponível na Web.

Três sublinguagens provêm diferentes níveis crescentes de expressividade da linguagem OWL, atendendo diferentes requisitos.

- OWL Lite atende principalmente requisitos de classificação hierárquica e restrições simples. OWL Lite estende a linguagem RDF, mas não usa todas as características da linguagem OWL;

- OWL-DL usa o vocabulário OWL completo. Contudo, OWL-DL está sujeita a algumas restrições. OWL-DL visa obter o máximo de expressividade, sem comprometer a completeza e decidibilidade. Isso significa, respectivamente, garantir que todas as conclusões são computáveis e que são realizadas em um tempo finito;

- OWL full oferece expressividade máxima somada à liberdade sintática da linguagem RDF, sem oferecer qualquer garantia computacional. OWL full pode ser entendida como uma extensão irrestrita de RDF, enquanto OWL$D L$ pode ser vista como uma extensão restrita de RDF com expressividade 
maximizada. Os próximos parágrafos descrevem características relevantes das sublinguagens de OWL.

Cada sublinguagem é uma extensão da anterior. OWL Full é uma extensão de RDF, enquanto que as outras são extensões de um subconjunto de RDF. Em cada aplicação escolhe-se a mais adequada, de acordo com os parâmetros expressividade e computabilidade.

\section{- Características herdadas de RDF-S}

Class: uma classe define um grupo de indivíduos que compartilham um conjunto de propriedades. Cada indivíduo de uma classe é denominado instância da classe e o conjunto de indivíduos associados a uma classe é denominado extensão da classe. O significado intencional da classe está relacionado, mas não é igual à extensão da classe. Desse modo, duas classes diferentes podem ter a mesma extensão. Classes em OWL podem ser descritas através da definição de um nome para a classe e pela adição de restrições sobre a extensão da classe, ou simplesmente declarando restrições sobre a extensão de uma classe anônima. A classe Thing é a classe raiz da hierarquia de classes OWL. Todo indivíduo em uma base de conhecimento é uma instância da classe Thing.

Individual: indivíduos são instâncias de classes e se relacionam entre si através de propriedades.

rdfs:subClass Of : usada para declarar que uma classe é subclasse de uma outra classe e para criar uma hierarquia de classes;

rdf:Property: usada para estabelecer relacionamentos entre indivíduos (ObjectProperty) ou de indivíduos para valores de dados (DatatypeProperty);

rdfs:subPropertyOf : declara que uma propriedade é subpropriedade de outra, criando uma hierarquia de propriedades e possibilitando inferir que um indivíduo relacionado através de uma propriedade também o será de uma através de outra propriedade da qual a primeira foi declarada subpropriedade;

rdfs:domain: o domínio de uma propriedade limita os indivíduos para o quais a propriedade pode ser aplicada. Declarar uma classe como domínio de uma 
propriedade implica que um indivíduo que se relaciona com outro através dessa propriedade, pertence à classe que foi declarada domínio dessa mesma propriedade;

rdfs:range: a imagem de uma propriedade limita os indivíduos que a propriedade pode ter como valores. Se uma classe é declarada imagem de uma propriedade e um indivíduo se relaciona com outro, através dessa propriedade; então, o outro indivíduo pertence à classe que foi declarada imagem dessa mesma propriedade.

\section{- Igualdades e desigualdades - OWL Lite}

equivalentClass: usada para declarar classes sinônimas, implicando que classes equivalentes têm as mesmas instâncias.

equivalentProperty: usada para declarar propriedades sinônimas. Isso implica que indivíduos relacionados através de uma propriedade também o são através de propriedades sinônimas. Propriedades equivalentes também são subpropriedades uma da outra.

sameAs: usado para criar diferentes nomes para um mesmo indivíduo.

diferentFrom: estabelece que um indivíduo é diferente de outro indivíduo. Usada principalmente para explicitar que dois nomes não se aplicam à um mesmo indivíduo, reforçando a hipótese de "nome único ".

alIDiferent: pode ser entendida como uma extensão da característica anterior para explicitar que indivíduos em um grupo são mutuamente distintos.

\section{- Características de propriedades - OWL Lite}

Inverse Of : As propriedades têm um sentido, no caso da inversa, este sentido pode ser dado de ambos os lados, assim para cada par $\mathrm{P} 1(\mathrm{x}, \mathrm{y})$ existe um inverso P2 $(y, x)$ e vice-versa.

TransitiveProperty: Uma propriedade $\mathrm{R}$ é transitiva quando, se $\mathrm{x}$ está relacionado com y e y está relacionado com $z$, então $x$ se relaciona com $z$, assim: se, para cada $x, y, z \in E$; existe $R(x, y)$ e $R(y, z)$ implica $R(x, z)$. 
SymmetricProperty: Uma propriedade $R$ é simétrica quando, se $x$ se relaciona com $y$, y se relaciona com $x$, assim: se para cada $x, y \in E ; R(x, y)$ implica $\mathrm{R}(\mathrm{y}, \mathrm{x})$.

FunctionalProperty: Uma propriedade funcional é aquela em que só tem um único valor de y para cada instância de $X$. Portanto, não tem mais do que um único valor para cada indivíduo. Uma propriedade com esta característica tem cardinalidade mínima 0 e cardinalidade máxima 1.

InverseFunctionalProperty: declarar que uma propriedade é funcional inversa implica que a inversa da propriedade é funcional.

\section{- Restrições de quantificadores sobre propriedades - OWL Lite}

Descrevem ou limitam o conjunto de valores possíveis para uma propriedade. Cada propriedade é modelada por um conjunto de restrições que definem os valores que podem ser assumidos, os quais descrevem ou limitam o conjunto de valores possíveis para uma propriedade.

allValuesFrom: A restrição allValuesFrom é declarada em uma propriedade com respeito a uma classe, isto é, a restrição requer que, para cada instância da classe que tenha instâncias da propriedade especificada, os valores da propriedade devem ser todos os membros da classe indicada pela cláusula allValuesFrom.

someValuesFrom: A restrição someValuesFrom descreve a classe de indivíduos $\mathrm{x}$ para os quais existe pelo menos um $\mathrm{y}$, tal que o par $(\mathrm{x}, \mathrm{y})$ seja uma instância da propriedade, isto é, a restrição requer que, pelo menos para uma instância da classe que tenha instâncias da propriedade especificada, os valores da propriedade devem ser membros da classe indicada pela cláusula someValuesFrom.

\section{- Restrições de Cardinalidade - OWL Lite}

A OWL permite utilizar restrições de cardinalidade, uma vez que qualquer instância de uma classe pode ter um número arbitrário de valores para uma propriedade. As restrições de cardinalidade são restrições locais, uma vez que elas são declaradas em propriedades com respeito a uma classe. Segundo (BECHHOFER et al., 2004), a OWL provê três construções para cardinalidade: 
minCardinality: definir cardinalidade mínima igual a 1, para uma propriedade em relação a uma classe, equivale a exigir um valor para essa propriedade para todas as instâncias dessa classe; definir cardinalidade mínima zero implica, na ausência de informação mais específica, que a propriedade é opcional em relação à classe.

maxCardinality: definir cardinalidade máxima igual a 1, para uma propriedade em relação à uma classe, implica que toda instância dessa classe se relaciona no máximo a um indivíduo através dessa propriedade; definir cardinalidade máxima zero estabelece que a classe não possui valor para aquela propriedade.

cardinality: serve para definir cardinalidade mínimas e máximas iguais a zero, ou iguais a 1, para uma propriedade em relação a uma classe.

\section{- Classe de interseção - OWL Lite}

IntersectionOf : usada para declarar intersecções de classes com nome e restrições. Por exemplo, a classe BSPLibrary pode ser descrita como intersecção da classe Library que se relaciona com a classe BSPModel através da propriedade implementsModel.

\section{- Datatypes - OWL Lite}

Os tipos de dados OWL são emprestados de RDF e XML Schema. Mais detalhes sobre Datatypes podem ser encontrados em (BECHHOFER et al., 2004).

\section{- Headers - OWL Lite}

Os cabeçalhos das ontologias aceitam inclusão de ontologias, relacionamentos, e informações gerais sobre a ontologia.

\section{- Annotations - OWL Lite}

Indivíduos, propriedades, classes e cabeçalhos das ontologias podem receber anotações; contudo, essas anotações sofrem algumas restrições, não podendo, por exemplo, ser usadas em axiomas de propriedades, tais como subpropriedades e restrições de domínio e imagem. 
- Versions - OWL Lite

As declarações owl: versionInfo, owl:priorVersion, owl:backwardCompatibleWith, owl:incompatibleWith, owl:deprecatedClass, e owl:deprecated:Property permitem monitorar a evolução das ontologias OWL.

Informações detalhadas sobre as características declaradas acima são encontradas na referência (BECHHOFER et al., 2004).

\section{- Características OWL-DL e OWL-full}

oneOf : usado para descrever classes através da enumeração de indivíduos; os membros da classe são exatamente o conjunto de indivíduos enumerados.

hasValue: A propriedade hasvalue permite que uma propriedade tenha um certo indivíduo como valor. Em outras palavras, hasvalue permite especificar classes baseadas na existência de valores de propriedades particulares, assim, um indivíduo será membro de tal classe sempre que pelo menos um dos valores das propriedades for igual ao valor do recurso hasvalue.

disjointWith: declara que classes são disjuntas entre si. Nesse caso, pode-se inferir estados inconsistentes se indivíduos forem declarados instâncias de um conjunto de classes disjuntas, ou ainda, se um indivíduo de uma certa classe não é instância de classes declaradas disjuntas da sua classe.

unionOf, complementof, intersectionOf: usadas para descrever combinações booleanas de classes e restrições.

minCardinality, maxCardinality, cardinality: estende as restrições de cardinalidade de OWL Lite permitindo declarações de cardinalidade para quaisquer valores inteiros não negativos.

Classes complexas: extensão OWL full para declaração arbitrária de classes complexas em diversas estruturas não permitidas em OWL Lite. Também em OWL full classes podem ser tratadas como indivíduos, isto é, instâncias de outras (meta) classes. 


\subsubsection{Ferramentas}

Existem diversas ferramentas para o tratamento de ontologias. Passaremos a descrever o Protégé, que foi a ferramenta utilizada neste trabalho, além de citarmos algumas outras ferramentas existentes.

O Protégé: (PROTÉGÉ, 2006) é um editor de ontologias open source baseado em Java, que inclui um framework de base de conhecimento. É um ambiente integrado utilizado por desenvolvedores de sistemas e pesquisadores em um domínio específico para desenvolver e manter uma ontologia. Através dele é possível descrever os conceitos pertencentes à ontologia, juntamente com seus atributos e relacionamentos.

O Protégé pode ser utilizado como um plugin do Eclipse ${ }^{4}$ em qualquer máquina que tenha uma JDK instalada. Suporta qualquer banco de dados que tenha driver JDBC como: Oracle, MySQL, Microsoft SQL Server e Microsoft Access. Permite múltiplos usuários simultaneamente e tem uma Application Programming Interface (API) documentada para auxílio aos usuários.

Dentre os diversos plugins existentes e em desenvolvimento pela comunidade de usuários do Protégé, em nosso trabalho utilizamos o OntoViz Tab, que permite lidar com ontologias no Protégé através da ajuda de um sofisticado software para visualização em grafo (Graphviz da ATeT). A ferramenta dá a opção de selecionar um conjunto de classes e instâncias para visualizar partes de uma ontologia e especificar cores para nós do grafo e outros elementos.

Além de plugins para modificações na interface com o usuário, a arquitetura do Protégé permite backend plugins que lêem e escrevem em diferentes formatos de armazenamento. Como padrão, a ferramenta armazena suas bases de conhecimento em um formato de arquivo de propósito especial. Atualmente podem ser criadas classes e instâncias em CLIPS, OIL, Jess, XML, RDF, F-Logic, Prolog, Topic Maps (linguagem padrão ISO para descrever estruturas de conhecimento em

${ }^{4}$ O Eclipse é um ambiente integrado de desenvolvimento (IDE) feito em Java e pode ser utilizado com diferentes linguagens de programação: $C, C_{++}$, Java, PHP, Ruby, Phyton e até $C O B O L$ 
páginas da Web), além do armazenamento em bancos de dados relacionais. Essa última capacidade é fundamental para grandes bases de conhecimento, que excedem o tamanho físico da memória principal e precisam de uma maneira eficiente de recuperar a informação de uma memória secundária.

OntoEdit: (MIZOGUCHI, 2004) é um ambiente gráfico de desenvolvimento para projeto e manutenção de ontologias. Possui uma máquina de inferência chamada FaCT. O processo de desenvolvimento de ontologia no OntoEdit é baseado em uma metodologia própria denominada On-To-Knowledge. O sucessor do OntoEdit é o OntoStudio (ONTOPRISE, 2008), que é baseado no ambiente de desenvolvimento Eclipse, da IBM, e está disponível nas versões: profissional e livre (para uso não comercial). As linguagens suportadas são: OWL e RDF, além de Flogic e OXML. Estas últimas são linguagens otimizadas para processamento de ontologias baseados em lógica.

DOE: (The Diferential Ontology Editor) (ISSAC; TRONCY, 2006) é um editor simples para criação de ontologias. No DOE são construídas as taxonomias dos conceitos e relações, justificando explicitamente a posição de cada item na hierarquia. Feito isto, o usuário pode então adicionar sinônimos e definições. Consideram-se duas taxonomias do ponto de vista semântico extensional. O usuário poderá aumentá-las ou adicionar restrições às relações. A ontologia pode ser traduzida em uma linguagem de representação do conhecimento. As linguagens disponibilizadas são: RDFS, OWL, DAML+OIL, OIL, linguagem para especificação de gráficos conceituais (CGXML).

Chimaera (CHIMAERA, 2005) é um sistema de software que permite aos usuários criar e manter ontologias distribuídas na Web. As duas maiores funções que ele suporta são mesclagem de múltiplas ontologias e diagnóstico de várias ontologias ou individualmente. Também permite o carregamento de bases de conhecimento em diferentes formatos, reorganização de taxonomias, resolução de conflitos de nome, edição de termos, entre outras opções. O Chimaera está disponível para uso na Internet, solicitando registro para acesso completo à sua versão funcional. Ele pode carregar e exportar arquivos no formato OWL e DAML. 


\subsection{Mecanismos de raciocínio}

Os raciocinadores ou reasoners são capazes de fazer inferências sobre objetos de dados utilizando a informação de seus relacionamentos definidos através de uma ontologia, ou seja, verifica se novas informações podem ser derivadas de uma ontologia. Nesta dissertação foi utilizado o Pellet, que será citado a seguir, juntamente com outros raciocinadores:

Pellet: (PELLET, 2006) e (SIRIN; PARSIA, 2004) é um software de código aberto feito em Java usando o OWL e baseado no tipo de lógica descritiva (descrição lógica ou DL).

Pellet atende as recomendações $W 3 C$ de casos de testes definidas em (CARROLL et al., 2004), e (SIRIN; PARSIA, 2004), para verificadores de documentos OWL:

1) verificação de sintaxe $O W L$;

2) verificação de consistência OWL para OWL Lite, OWL-DL e OWL full;

3) verificação completa de consistência $O W L$ para $O W L$ Lite. Uma verificação completa é decidível em relação à semântica.

Pellet é um verificador sintático e um verificador de consistência completo, isto é, semanticamente decidível para OWL-DL.

Pellet integra a verificação de consistência ao seguinte conjunto padrão de serviços de inferência lógica, os quais podem ser acessados através de consultas:

- Verificação de consistência - assegura que uma ontologia não contém fatos contraditórios;

- satisfazibilidade de conceito - verifica se é possível a uma classe (conceito) possuir instâncias;

- classificação - calcula as relações de sub-classe existentes entre cada classe nomeada para criar a hierarquia de classes;

- realização - define a classe mais específica (direta) à qual um indivíduo pertence. 
O conjunto completo de funcionalidades e características de Pellet inclui a realização de consultas usando linguagens como SPARQL. Pellet também realiza verificação sintática automática através de heurísticas que tentarão transformar documentos $O W L$ full em $O W L-D L$ e então, processá-los normalmente. Na realização de detalhamento e depuração de ontologias, Pellet considera a totalidade de características OWL-DL (propriedades inversas e transitivas, raciocínio sobre tipos de dados, restrições de cardinalidade, classes enumeradas e declarações de instâncias).

A arquitetura de Pellet é centrada em torno de um pequeno motor de inferência adaptado para sofrer extensões. Esse modelo permitiu o desenvolvimento de interfaces para diferentes ferramentas OWL/RDF, tais como Jena, descrita em (CARROLL et al., 2004) e WonderWeb OWL API apresentada em (BECHHOFER; LORD; VOLZ, 2003), ou para apoiar aplicações que se comunicam através da interface DIG, descrita em (BECHHOFER; MOLLER; CROWTHER, 2003). Pellet provê diferentes interfaces para carga de ontologias e também implementar interfaces de raciocínio, definidas pelas ferramentas de extensão, para responder consultas. Existem outros mecanismos de inferência apresentados em (ZOU, 2004):

Racer: o Racer (RACER SYSTEM, 2006) é um sistema de representação de conhecimento baseado em DL. O sistema provê suporte para especificações de terminologias em geral, através do uso de axiomas, inclusive axiomas que descrevam a relação hierárquica entre conceitos, definições múltiplas ou cíclicas. $O$ sistema também provê a verificação de consistência, além de várias funções e serviços de recuperação de informação pré-programados.

FaCT: o sistema FaCT (HORROCKS, 2006) é um classificador de DL que também pode ser usado para teste de satisfabilidade em lógica modal. Além de implementar uma DL com alto grau de expressividade, o sistema provê suporte para raciocínio envolvendo bases de conhecimento que contenham axiomas gerais de inclusão de conceitos. 


\subsection{Mecanismos de Recuperação de informações}

Nesta seção, discutiremos a utilização de duas diferentes abordagens para o problema de recuperação de informações em bases de conhecimento descritas em $O W L-D L$. Ambas as estratégias partem da necessidade da realização de consultas sobre as bases de conhecimento.

A primeira abordagem discute a utilização de uma ferramenta de inferência associada à uma linguagem de consulta simples. Especificamente, descrevemos características relevantes da linguagem de consulta SPARQL, apresentada em (DODDS, 2006) e (MCCARTHY, 2005).

Linguagem de consulta OWL-QL é o ponto de partida da segunda abordagem. OWL Query Language (OWL-QL) é uma linguagem de consulta para bases de conhecimento em OWL. OWL-QL possibilita a realização de diálogos baseados em consulta e resposta que podem ser automatizados através de métodos de raciocínio. Serão apresentadas características relevantes de OWL-QL e ferramentas de consulta baseadas em inferência usando OWL-QL.

\subsubsection{A Linguagem SPARQL}

A linguagem de consulta SPARQL (CONSORTIUM W.W.W.W., 2007) é baseada na semelhança de padrões de grafos e é em termos do modelo RDF, definido pelo consórcio W3C, funcionando para qualquer fonte de dados que possa ser mapeada em RDF. SPARQL é ao mesmo tempo uma linguagem de consulta e um protocolo de acesso. Diversas ferramentas e APIs são disponibilizadas para prover funcionalidade baseada em SPARQL. Uma pequena lista disponível em (DODDS, 2006) inclui, entre outros, ARQ, um processador SPARQL para Jena; KAON2, um mecanismo de inferência com suporte parcial à consultas em SPARQL e Pellet, o mecanismo Java de código aberto, descrito anteriormente.

SPARQL, assim como RDF, é construída sobre uma tripla consistindo de sujeito, predicado e objeto. Uma tripla padrão SPARQL pode incluir variáveis e estas 
são usadas para indicar itens de dados de interesse que resultarão de uma consulta. Sujeito, predicado e objeto, podem ser substituídos uma variável. Cada tripla que combina com o padrão agregarão um valor real do conjunto de dados RDF para cada variável.

O exemplo abaixo mostrado no

Quadro 1, emprestado de (DODDS, 2006) usa variáveis para sujeito e objeto, no caso element e name.

PREFIX Tabela: <http://www.daml.org/2003/01/periodicTabela/PeriodicTabela\#>

SELECT ?name

FROM <http://www.daml.org/2003/01/periodicTabela/PeriodicTabela.owl>

WHERE \{ ?element Tabela:name ?name. $\}$

\section{Quadro 1 Código exemplo de SPARQL}

A palavra chave PREFIX equivale em sua essência à definição de um espaço de nomes $X M L$. O rótulo atribuído a uma URI pode ser usado em qualquer lugar em uma consulta em substituição da própria URI. A cláusula SELECT é usada para definir os itens de dados que serão retornados pela consulta. A palavra chave FROM identifica os dados sobre os quais a consulta vai ser executada. Finalmente, a cláusula WHERE define o padrão da consulta, isto é, uma coleção de padrões de tripla que identifica a forma do grafo que se deseja obter na combinação resultante da consulta.

SPARQL permite o uso de várias triplas no padrão para construção de consultas mais sofisticadas e complexas. As consultas podem ser construídas a partir de dados incompletos ou ausentes e sobre combinações de alternativas. Adicionalmente, o resultado pode ser ordenado e ter seu tamanho limitado. 


\section{OWL-QL}

OWL-QL (FIKES; HAYES; HORROCKS, 2003) é uma linguagem formal e também um protocolo usado por agentes de consulta (clientes) e agentes de resposta (servidores) na condução de um diálogo do tipo "consulta e resposta" sobre uma Base de conhecimento representada em OWL. OWL-QL é uma atualização da linguagem de consulta $D A M L$ Query Language (DQL).

Nos diálogos de consulta e resposta em OWL-QL, o servidor pode usar métodos de raciocínio automáticos para derivar respostas e cenários nos quais o conhecimento usado para responder a consulta pode estar em múltiplas bases de conhecimento espalhados na Web semântica. Tais bases de conhecimento podem ou não ser especificadas pelo cliente. OWL-QL permite que o servidor defina as bases de dados a serem usadas em resposta a uma consulta e que o cliente peça ao servidor para identificar a base de conhecimento usada nas respostas. 


\section{Capítulo 4}

\section{Trabalhos Relacionados}

Os trabalhos descritos no presente capítulo foram selecionados em razão de sua relevância e da intersecção de seu escopo com o problema tratado pela nossa pesquisa. Conforme descrito no capítulo introdutório, nosso trabalho investiga uma abordagem semântica para o problema de gerenciamento de dados das RSSF.

As seções que se seguem apresentam uma análise comparativa entre o nosso trabalho e os trabalhos relacionados. As seções são organizadas com o objetivo de apresentar trabalhos, que compartilham aspectos semelhantes entre si.

\subsubsection{Propostas Recentes}

A proposta apresentada por Henricksen e Robinson (2006) no artigo "Middleware for sensor Networks: State-of-the-Art- and Future Directions" sugere que as novas soluções para os middleware de redes de sensores devam ser mais genéricas, suportem hardware heterogêneo e diversos mecanismos de comunicação. A contribuição deste trabalho foi a discussão de como o middleware para as redes de sensores pode ser combinado com sistemas cientes ao contexto.

Em geral, os middleware para sistemas cientes ao contexto suportam consultas e técnicas de fusão dos dados; por exemplo, em reconhecimento facial, estes sistemas possam responder as perguntas como "quem está no frame atual?". Estes middleware fazem uso de regras de raciocínio que permite inferir nova informação, abstraída da informação recolhida, por exemplo, dado um conjunto de regras apropriadas ou de algoritmos de aprendizagem, é possível obter com algum grau de exatidão a atividade e posição de uma pessoa em um tempo determinado. Por outro lado, as soluções do middleware para redes do sensor fornecem tipicamente consultas simples, como mínimo, máximo, soma média, etc. 
O foco do trabalho descrito em (HENRICKSEN; ROBINSON, 2006) são os avanços na área de sistemas cientes ao contexto; relacionando-os ao modelo de raciocínio sobre dados derivados dos sensores, modelando a qualidade de informação de detecção para resolver conflitos ou inconsistência da informação. Nossa proposta tem seu foco na criação de uma estrutura semântica baseada em ontologias para interagir com os dados dos sensores e informação do contexto do ambiente.

A proposta apresentada em (BALAZINKA; DESHPANDE, 2007) no artigo "Data Management in the worldwide sensor web" realizada por várias universidades em conjunto, elas propõem o gerenciamento de dados para uma grande quantidade de redes de sensores por intermédio da Internet, como se mostra na Figura 5. As redes de sensores de toda índole, como por exemplo, captura de vídeo para monitorar reservas de animais selvagens, ou sensores biológicos implantados no corpo para monitorar sinais vitais, gera grandes volumes de dados que precisam ser gerenciados. A maioria dos pesquisadores de redes de sensores concordaria que se dá mais atenção ao trabalho de detectar e distribuir os dados e pouca atenção nas ferramentas para controlar, analisar e compreender os dados.

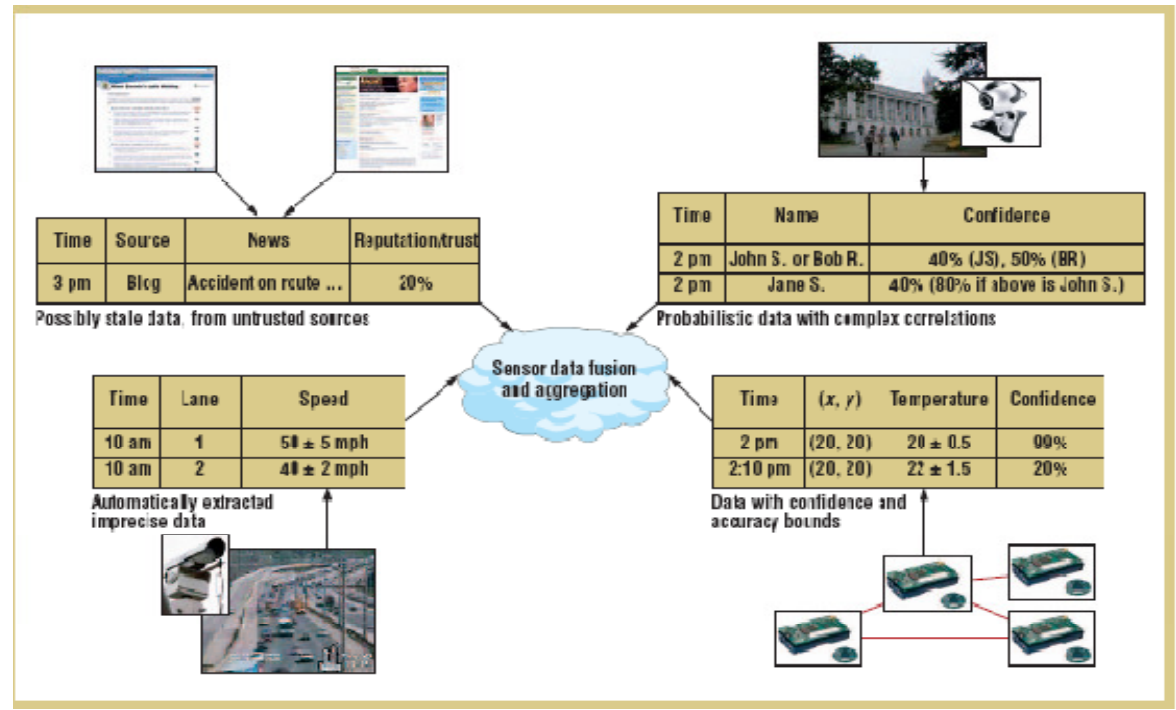

Figura 5 Gerenciamento de dados no worldwide sensor web (BALAZINKA; DESHPANDE, 2007)

Balazinska et Al (2007), propôs uma arquitetura para o gerenciamento de dados; ele divide esta arquitetura em: 
- Controle temporal e espacial; no caso do controle espacial refere-se a dados que são representadas facilmente, como por exemplo, coordenadas geográficas (latitude e longitude);

- Análise de exploração e visualização; implica ao analista conhecer a origem dos dados ou poder identificá-los através de processos de descoberta. Uma modelagem estatística de dados do sensor pode ser uma solução, mas infelizmente os gerenciamentos de dados atuais não suportam modelos estatísticos, forçando aos usuários a empregar ferramentas externas para esta finalidade. Como exemplo, os usuários importam os dados em pacotes para só depois fazer a análise em softwares específicos como: Matlab, $\mathrm{SAS}^{5}$, ArcGIS ${ }^{6}$, entre outras;

- Incerteza dos dados; refere-se à manipulação de dados incertos, esta pesquisa utiliza a teoria da probabilidade como base para representar a incerteza;

- Interoperabilidade dos dados; pode-se comparar ou combinar dados de muitas fontes heterogêneas mantidas por entidades independentes;

- Distribuição de dados; os dados do sensor são armazenados perto de sua fonte, mas as consultas podem ser em qualquer lugar do mundo. Freqüentemente, a maior consulta de dados de interesse está na mesma região geográfica;

- E, finalmente, privacidade e segurança de dados.

A proposta apresentada por Balazinska et al. (2007), é uma das motivações de nosso trabalho, já que sugere uma possibilidade interessante de sua exploração na definição de semântica no gerenciamento de dados dos sensores. Parte desta proposta é considerada em nosso trabalho, como a interoperabilidade e distribuição de dados.

\footnotetext{
${ }^{5}$ SAS.-é um Sistema de Analises estatístico.

${ }^{6}$ ArcGIS .- é o nome de um grupo de programas informáticos e que constitui um Sistema de informação geográfica.
} 


\subsubsection{Aplicações Desenvolvidas}

No artigo "A Semantic Approach to Data Management in Sensor Networks" desenvolvido por (LEWIS et al., 2006), apresenta-se uma ferramenta de gerenciamento dos dados chamada ES3N onde utiliza técnicas de web semântica para controlar e consultar dados coletados de uma rede de sensores, capturando associações importantes entre estes dados e armazenando em uma ontologia dinâmica distribuída.

Este trabalho mostra as potencialidades da gerência de dados de redes de sensores homogêneos e heterogêneos. Para a elaboração deste projeto usou-se Protégé 3.2, que é um editor de ontologia open source, exportado facilmente para formatos OWL e de RDF/RDFs. A schema da ontologia consiste em um conjunto de classes predefinidas, atributos e arquivos exportados como OWL.

Neste trabalho, os autores demonstram que as técnicas semânticas são importantes para obter associações entre os dados deste modo adiciona potencialidade às consultas. Propõe-se uma ontologia que ofereça a recuperação rápida e eficiente de dados, mediante o uso de registros simples como se mostra na Figura 6. Nosso trabalho, comparativamente, propõe uma abordagem mais geral para o gerenciamento do conteúdo da rede de sensores. Espera-se alcançar um nível maior de generalidade e, conseqüentemente, um nível maior de reutilização. 


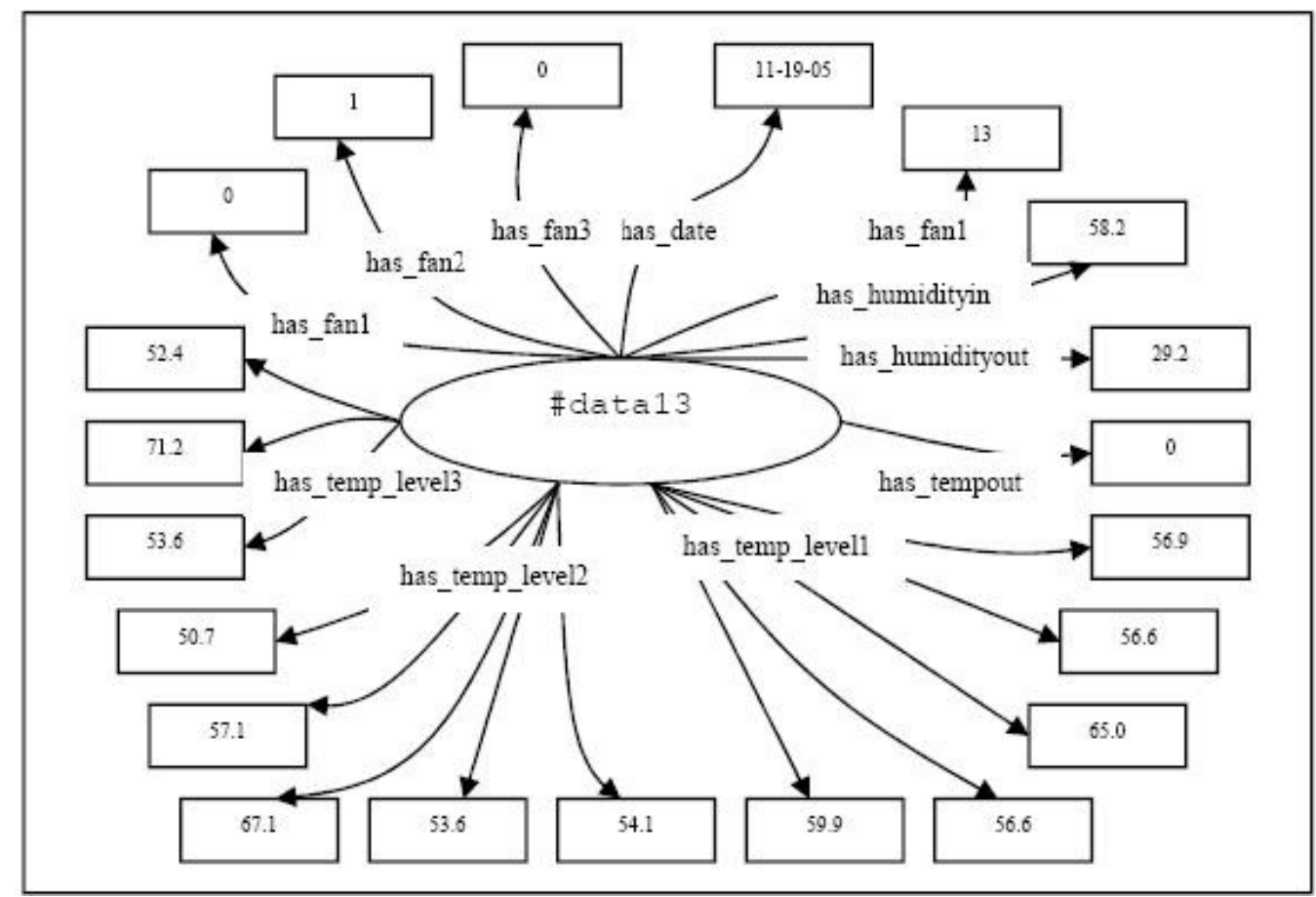

Figura 6 Registro Simples da Ontologia ES3N (LEWIS et al., 2006)

O Projeto descrito em (O'DONOGHUE; HERBERT, 2006d) "Data Managment System: A Contex Aware Architecture For Pervasive Patient Monitoring" apresenta uma arquitetura chamada (Data Management System) DMS que fornece um serviço ciente do contexto dentro de um ambiente médico. Uma das características essenciais de um ambiente médico é a aquisição em tempo real da informação e a entrega apropriada dos dados exatos.

Esta pesquisa foi conduzida em um número de áreas relacionadas incluindo a recuperação contextual dos dados médicos e dos ambientes médicos, baseados em agentes. Destaca-se, no trabalho a importância dos agentes, já que podem ser uma ferramenta útil para solucionar problemas de gerenciamento de dados em um ambiente médico, pois o nível da intercomunicação entre a equipe de funcionários, médicos e pacientes é elevado.

Alguns dos aspectos chaves de um sistema de gerência de dados em um ambiente médico (DMS) é apresentado no artigo "A QoS Data Management System within a Pervasive Medical environment" (O'DONOGHUE e HERBERT, 2006b) cujos 
Protocolos de QoS são : perfil de usuário, prioridade de dados, consistência de dados, avaliação de dados. Cada protocolo é executado sobre uma plataforma de agente inteligente; isto ativa o procedimento reativo e proativo dos agentes, baseado em todas as variáveis cientes do contexto disponíveis.

O foco da abordagem de DMS sobre monitoramento de pacientes constitui o principal diferencial em relação a nossa proposta. Nosso trabalho propõe uma abordagem mais geral, definida sobre um conjunto extensível de ontologias e metadados para o gerenciamento de dados relacionado aos diferentes domínios nas RSSF.

\subsubsection{Projetos}

O trabalho apresentado em (EID; LISCANO; SADDIK, 2007) no artigo "A Universal Ontology for Sensor Networks Data" propõe uma nova ontologia para recuperar dados relevantes do sensor, composta por representações dos termos ${ }^{7} \mathrm{e}$ definições de relacionamentos definidas pela ontologia. Além disso, estes relacionamentos podem ser usados para capturar sinônimos dos termos para recuperar toda a informação disponível para um conceito particular.

A metodologia usada para a criação desta ontologia é: a obtenção do vocabulário inicial, baseado nas linguagens de descrição de transdutores. A identificação da taxonomia inicial mostrada na Figura 7, as propriedades, restrições e prova da consistência. Foi implementada em Protégé OWL-DL como linguagem de representação de conhecimento e para a validação é usado RacerPro por ter interoperabilidade com Protégé.

\footnotetext{
${ }^{7}$ Termo é qualquer elemento constitutivo de uma expressão. A origem destes elementos no domínio de sensores foi IEEE 1451.4 standard family uses Transducer Electronic Data Sheet (TEDS).
} 


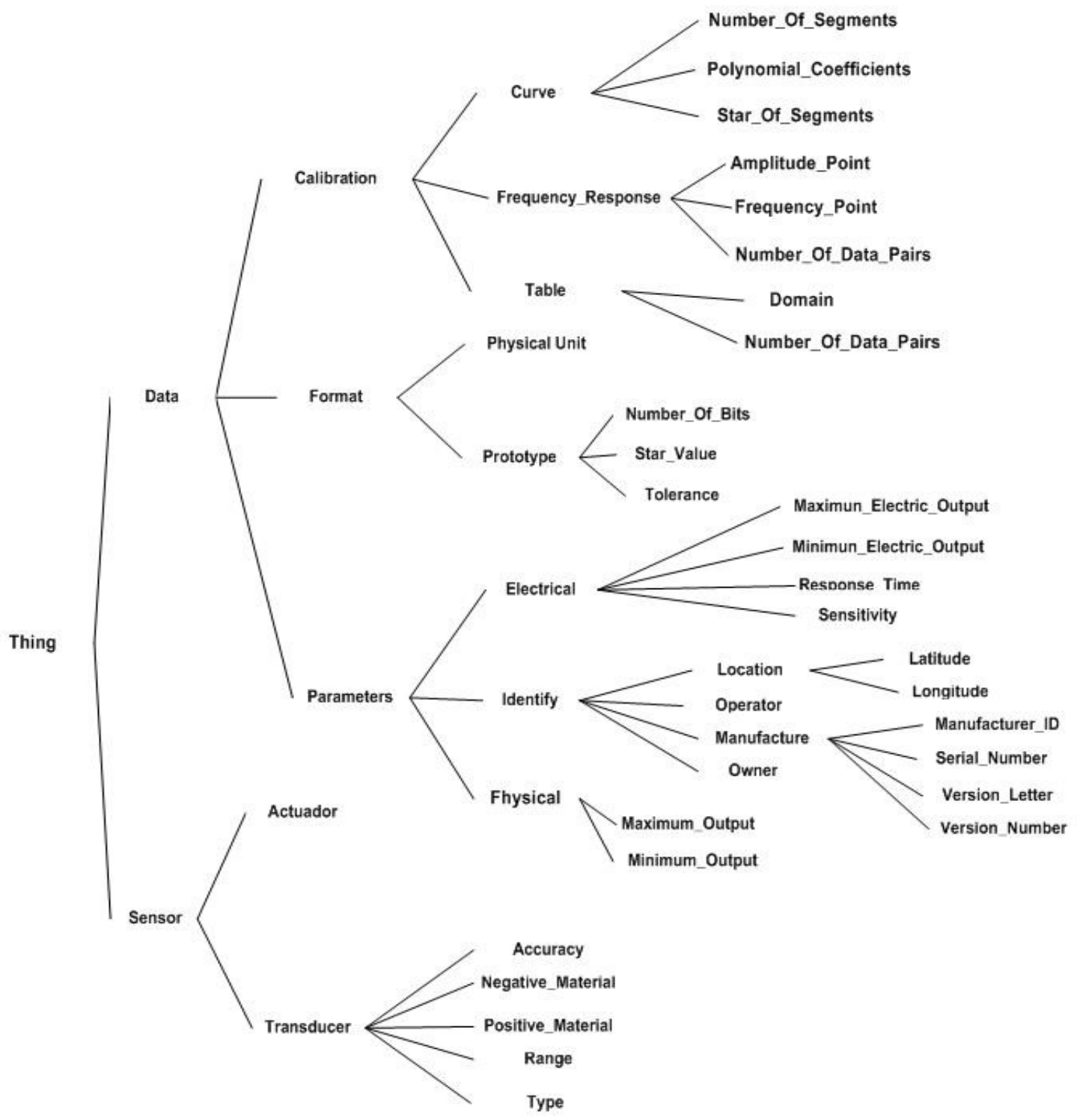

Figura 7 Taxonomia inicial da ontologia para dados do sensor (EID; LISCANO; SADDIK, 2007)

Esta ontologia propõe o uso do IEEE Suggested Upper Merged Ontology $\left(\mathrm{SUMO}^{8}\right)$, e a linguagem RDQL para realizar as consultas. Esta ontologia foi avaliada em uma aplicação de dados heterogêneos e distribuídos, o interesse deste trabalho é maximizar a precisão da procura de dados do sensor utilizando a informação semântica.

${ }^{8}$ IEEE SUMO é uma ontologia de propósito geral que está sendo desenvolvida por um grupo de trabalho da IEEE (Standard Upper Ontology Working Group). Está sendo utilizadas em pesquisas, aplicações de busca, processamento de linguagens naturais, entre outros. 
Este trabalho explora especificamente o domínio da ontologia para os dados de rede de sensores. Nosso trabalho, apesar de ter características semelhantes, é voltado para situações de integrar vários aspectos das RSSF através de ontologias e mecanismos semânticos.

O projeto Ontosensor (RUSSOMANNO; KOTHARI; THOMAS, 2005) é um repositório de conhecimento de sensores compatível com uma estrutura que envolve web semântica, como se mostra na Figura 8. Ontosensor inclui definições, conceitos e propriedades adotados desde SensorML, extensões para IEEE SUMO e referências para International Standards Organization ISO 19115.

As consultas foram desenvolvidas e testadas usando Protégé 2000 e Prolog. Propõe-se que Ontosensor possa servir como um componente nas aplicações que incluem mecanismos de inferência avançados, tais aplicações serão usadas para a fusão de dados heterogêneos em um ambiente centrado na rede (network-centric).

SensorML é especificado como um modelo genérico de dados em UML para capturar as classes e associações que são comuns a todos os sensores; foi realizado usando a sintaxe Extensible Markup Languaje(XML). SensorML é parte de uma iniciativa do Open Geospatial Consortium (OGC) para contribuir no desenvolvimento de sensor web, de modo que as aplicações e serviços possam abranger sensores de diferentes tipos sobre a web.

OWL foi adotado pelo consócio W3C como um padrão para a web semântica, cada construção de OWL tem uma semântica formal; parte do desenvolvimento de Ontosensor inclui traçar um subconjunto dos conceitos e das relações de SensorML para OWL.

Nossa proposta é semelhante à Ontosensor no que diz respeito à construção de uma ontologia genérica. Em comparação, o foco do nosso trabalho é sobre a identificação e descrição de cenários e mecanismos extensíveis para explorar a capacidade de inferência do modelo semântico, esperamos que o modelo resultante pudesse ser utilizado por diferentes sistemas de RSSF. 


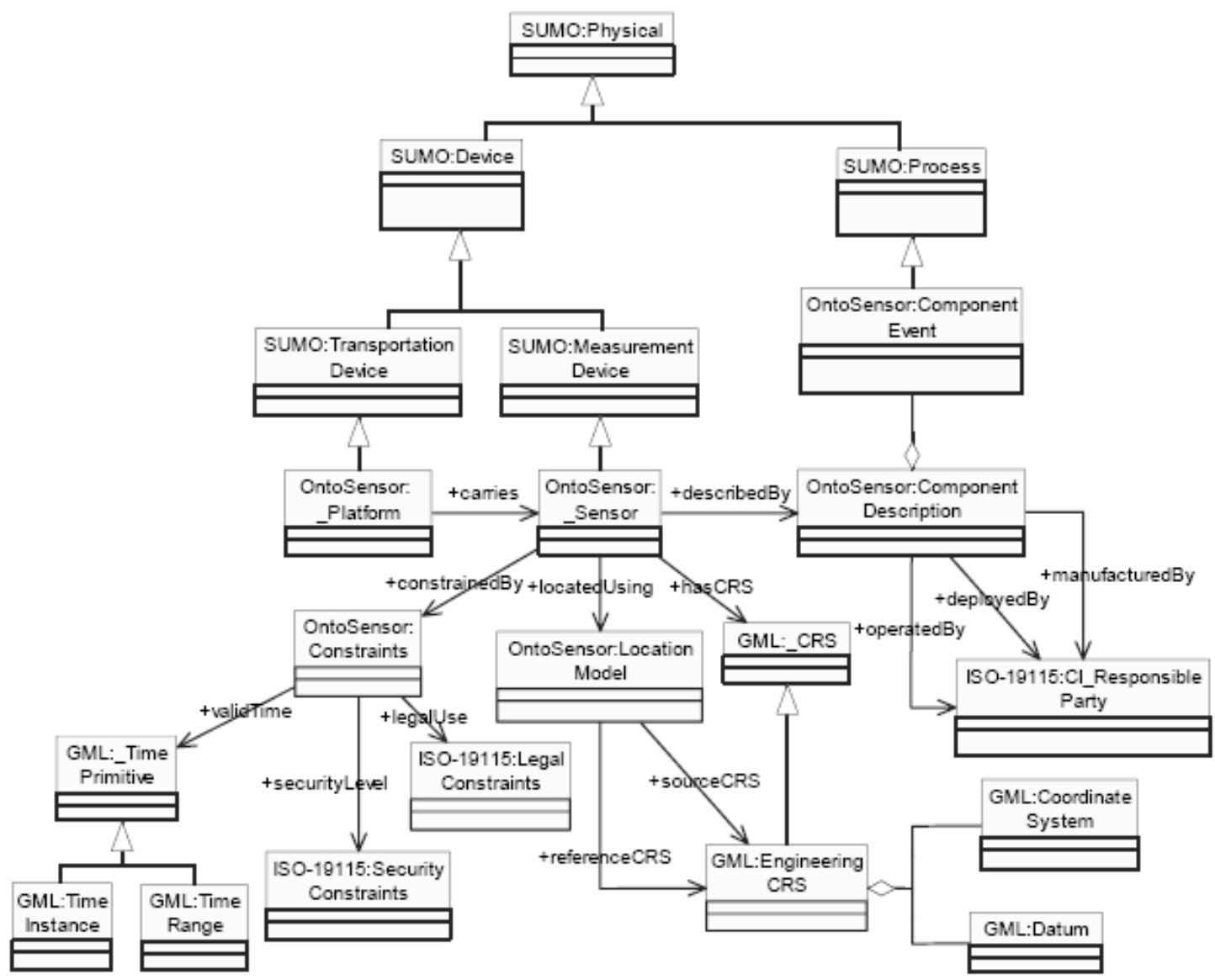

Figura 8 OntoSensor extensão de IEEE SUMO (RUSSOMANNO; KOTHARI; THOMAS, 2005)

MSRSense (CHEONG et al., 2005) é um projeto composto por uma coleção de ferramentas de software que permite que os usuários coletem, processem, arquivem, e visualizem dados de uma rede do sensor desde um MicroServer ${ }^{9}$.

Esta ferramenta coleta os dados em Microsoft Excel para depois fazer cálculos, análise e visualizações, Provê os dados em tempo real, permite o armazenamento de dados históricos e agregação de dados, como se mostra na Figura 9.

\footnotetext{
${ }^{9}$ MicroServer é um executável construído em .NET 2.0 encarregado de converter os packets TinyOS a packets $X M L$.
} 


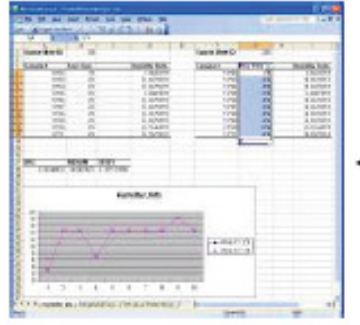

Excel

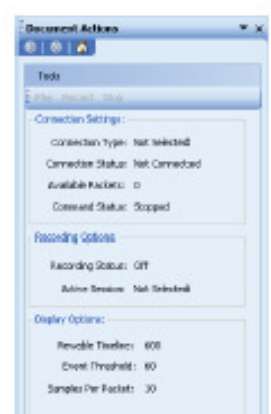

Packet Stream Player

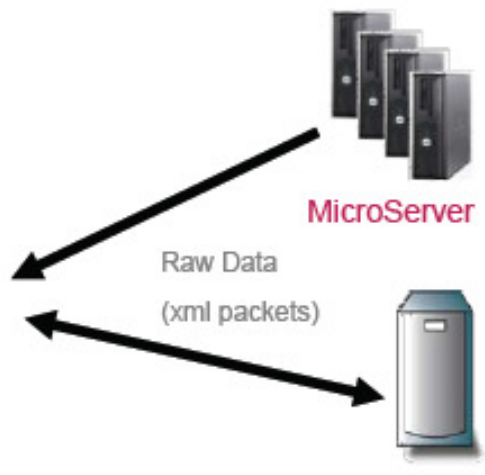

SQL 2005

Figura 9 MSR Sense (CHEONG et al. 2005)

O armazenamento dos dados é feito na DBMS SQL Server 2005, que permite a recuperação de informação. Nosso trabalho propõe uma contribuição a esta, já que uma geração nova de DBMS requer sustentações semânticas adicionais para uma gerência de dados flexível e eficiente. Isto inclui facilidades para a integração dos dados, otimização e processamento significativo da consulta. A idéia básica é dar à DBMS a habilidade de tratar as consultas tanto no nível semântico como no nível sintático. Se um usuário tentasse recuperar a informação sobre objetos de banco de dados, a resposta à sua consulta poderia não cumprir com suas expectativas. 


\section{Capítulo 5}

\section{Ontologias para o Gerenciamento de dados}

Este capítulo trata da concepção e definição do conjunto de ontologias que irão compor a estrutura semântica para o gerenciamento de dados das RSSF. Essas são as primeiras atividades relacionadas à implementação efetiva da proposta da dissertação.

Os conceitos, propriedades e axiomas definidos nas ontologias devem refletir os conceitos tratados nos capítulos de fundamentação teórica. O Capítulo 2 será a fonte de grande parte dos conceitos e relacionamentos descritos pelas ontologias. $O$ Capítulo 3 contribui com os conceitos, linguagens e ferramentas usadas na construção das próprias ontologias.

Neste Capítulo, descreve-se o conjunto de ontologias da base semântica proposta, essa descrição inclui classes, propriedades e axiomas que representam o domínio tratado. Decisões e definições implícitas no modelo também são discutidas.

\subsection{Desenvolvimento de ontologias para RSSF}

Existe atualmente um conjunto considerável de metodologias propostas para o desenvolvimento de ontologias. Corcho; Fernandez e Perez (2003) apresentam uma ampla revisão e comparação das principais metodologias, ferramentas e linguagens para desenvolvimento de ontologias. Cada uma segue diferentes abordagens, mas a maioria das metodologias analisadas propõe a divisão do desenvolvimento das ontologias em etapas.

Nossa escolha pela metodologia proposta em (NOY; MCGUINNESS, 2001) foi definida no contexto de um conjunto de decisões tomadas a partir da escolha da 
linguagem OWL, como linguagem para representação das ontologias que integram o nosso trabalho. A linguagem OWL, descrita no Capítulo 3, é uma linguagem padrão para representação de ontologias desenvolvida pelo (W3C) e largamente usada no contexto da Web semântica.

A escolha da linguagem para representação das ontologias determinou a adoção da ferramenta Protégé-OW (KNUBLAUCH et al., 2004), também descrita no Capítulo 3. Essa ferramenta permite o desenvolvimento de ontologias de modo incremental e confiável, principalmente graças a uma interface gráfica amigável e à conexão de motores de inferência para verificação de consistência das ontologias.

A metodologia descrita em (NOY; MCGUINNESS, 2001) é disponibilizada pelos seus desenvolvedores, no conjunto de ferramentas e documentação associadas ao Protégé-OWL e à linguagem OWL. Esse aspecto de confiabilidade e simplicidade da metodologia foram os dois fatores determinantes para a sua adoção.

A metodologia propõe a realização do seguinte conjunto de tarefas:

1. Determinar o Domínio e o Escopo da Ontologia:

- Definição do domínio e do escopo cobertos pela ontologia;

- Definição da finalidade da ontologia;

- Identificação dos tipos de questões para os quais a informação na ontologia deve prover respostas;

- Identificação dos atores responsáveis pelo uso e manutenção da ontologia.

2. Reutilizar Ontologias Existentes:

3. Levantar termos importantes:

4. Definir classes e sua hierarquia:

5. Definir propriedades das classes (slots):

6. Restrições das Propriedades (facetas):

7. Criação de instâncias: 
A aplicação dessa metodologia possibilita aos desenvolvedores da ontologia refletir sobre as necessidades reais de utilização da ontologia em um contexto. Em nosso trabalho, adotamos a metodologia como ponto de partida para o desenvolvimento do conjunto de ontologias para as RSSF.

As próximas subseções investigam, apresentam e analisam as definições e as identificações relacionadas nas quatro tarefas do primeiro ponto descrito acima, propostas pela metodologia adotada para o desenvolvimento das ontologias.

\subsubsection{Domínio e Escopo da Ontologia}

As ontologias para RSSF devem cobrir o domínio referente à integração de dados das redes de sensores. As classes da ontologia relacionam-se a conceitos como nós sensores, recursos de hardware, software, aplicações, dados coletados, dados da rede e os ambientes onde podem ser executados.

\subsubsection{Finalidade da ontologia}

Uma das principais finalidades da ontologia para RSSF é apoiar o gerenciamento e integração de diferentes dados de redes heterogêneas. Mais especificamente, a ontologia deverá facilitar a busca de conteúdo da rede, a seleção de recursos adequados para execução das aplicações na RSSF, integração de dados coletados e dados do ambiente.

A maioria das redes de sensores é construída para aplicações especificas, e um jogo de ontologias é definido como parte da estrutura para representar o conhecimento compartilhado do domínio de RSSF. 


\subsubsection{Questões Tratadas pela Ontologia}

Seguindo a metodologia, as definições do escopo e finalidade das ontologias para RSSF podem ser mais bem compreendidos com o apoio de um conjunto de questões que a base de conhecimento centrada na ontologia deve ser capaz de responder. No domínio analisado, a base de conhecimento deve responder a questões como as relacionadas nos exemplos abaixo.

- Que tipo de nó, quais são seus recursos de HW, SW e aplicação instalada?.

- Existe uma determinada aplicação adequada para execução em certa plataforma de nó?.

- Se existem diferentes redes, que nó sensor pertence a cada rede?.

- Quais são as leituras dos nós sensores?

- Qual é o estado atual do nó sensor? (voltagem, temperatura, LQI, etc.).

- A que grupo pertence o nó, origem, destino dos dados? (depende do frame do nó).

- Qual é o sinal físico do ambiente capturado pelo sensor? (temperatura, velocidade, aceleração, etc.).

- Qual é o tipo de aplicação, a quantidade de nós, que topologia se esta usando, que protocolo de comunicação? (depende da aplicação).

- Qual é a localização do nó e tipo de leitura? (depende da aplicação).

- Qual é o estado atual do ambiente monitorado?.

- Qual é a ação recomendada, segundo os dados capturados pelos nós?.

- Qual é a ação recomendada, de acordo com os critérios explicitamente definidos pelos recursos de funcionamento dos nós sensores?

- Qual é a ação recomendada, segundo os critérios das características do ambiente? Por exemplo, é um estado de alerta ou de emergência?.

A construção da ontologia e da base de conhecimento associada realiza-se em torno de questões dessa natureza. As perguntas acima são exemplos de exploração da semântica. A capacidade da base de conhecimento de respondê-las de modo eficiente deve legitimar sua utilidade. 


\subsubsection{Atores e casos de uso}

De acordo com a metodologia, a próxima atividade investiga os atores, papéis e casos de uso relacionados à manutenção e ao uso da base de semântica.

Analisamos apenas alguns atores e casos de usos considerados mais relevantes identificados no contexto de gerenciamento de dados das RSSF e seu conteúdo. Os modelos de casos de usos são mostrados em diagramas de UML (OMG, 2008), assim como uma descrição dos principais casos de usos para cada ator. $\mathrm{O}$ conjunto de atores considerados inclui:

\section{WSN ontology Developer}

Este ator representa uma abstração criada para descrever as atividades desenvolvidas na realização da nossa pesquisa, ele é o encarregado de criar e manter a ontologia proposta.

Criar a Ontologia: o ator WSN ontology Developer usa a ferramenta (ProtégéOWL) para a criação de um conjunto de ontologias sobre o domínio de RSSF. Classes, propriedades, restrições e instâncias de classes são criadas de modo incremental. A consistência do modelo deve ser verificada por alguma ferramenta de inferência (Racer, Pellet).

Suporte das Ontologias: visto que as ontologias são construídas para reuso, o WSN ontology Developer executa tarefas relacionadas mais especificamente à extensão, criação e eliminação de classes, propriedades e indivíduos, nas etapas finais do ciclo de desenvolvimento e manutenção das ontologias. A realização dessas tarefas deve preservar a coerência semântica do modelo.

Para os dois casos de usos a consistência base da ontologia também é definida no caso de uso do sistema "Fazer inferência".

A Figura 10 mostra o diagrama Unified Modelling Language (UML) e os casos de uso para esse ator. 


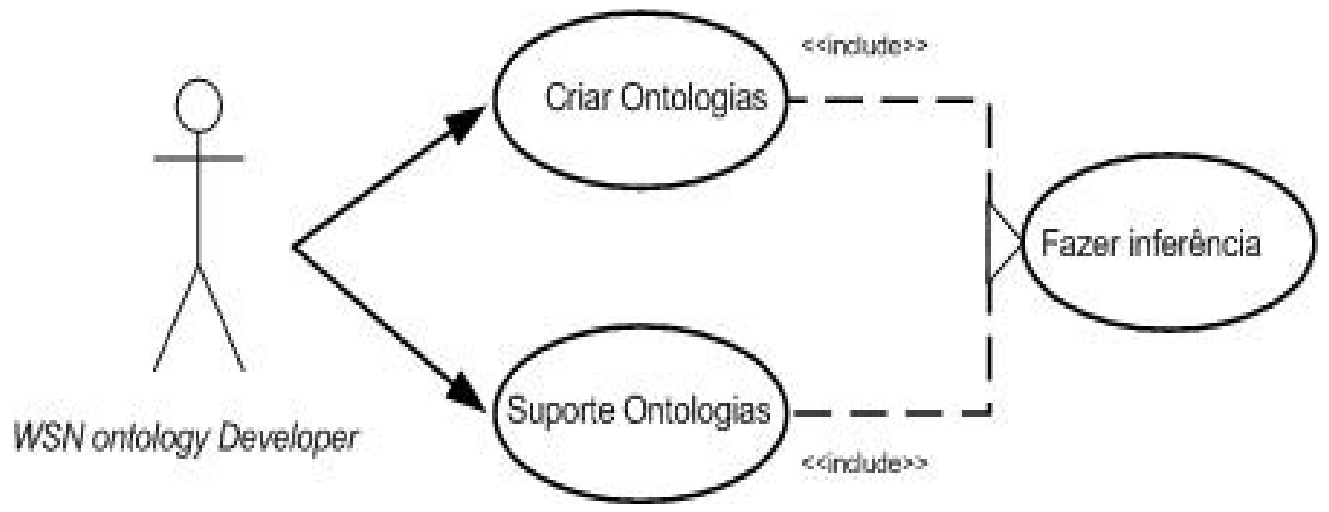

Figura 10 Casos de uso para o WSN ontology Developer

A Figura 11 apresenta a interface gráfica da ferramenta Protégé-OWL.

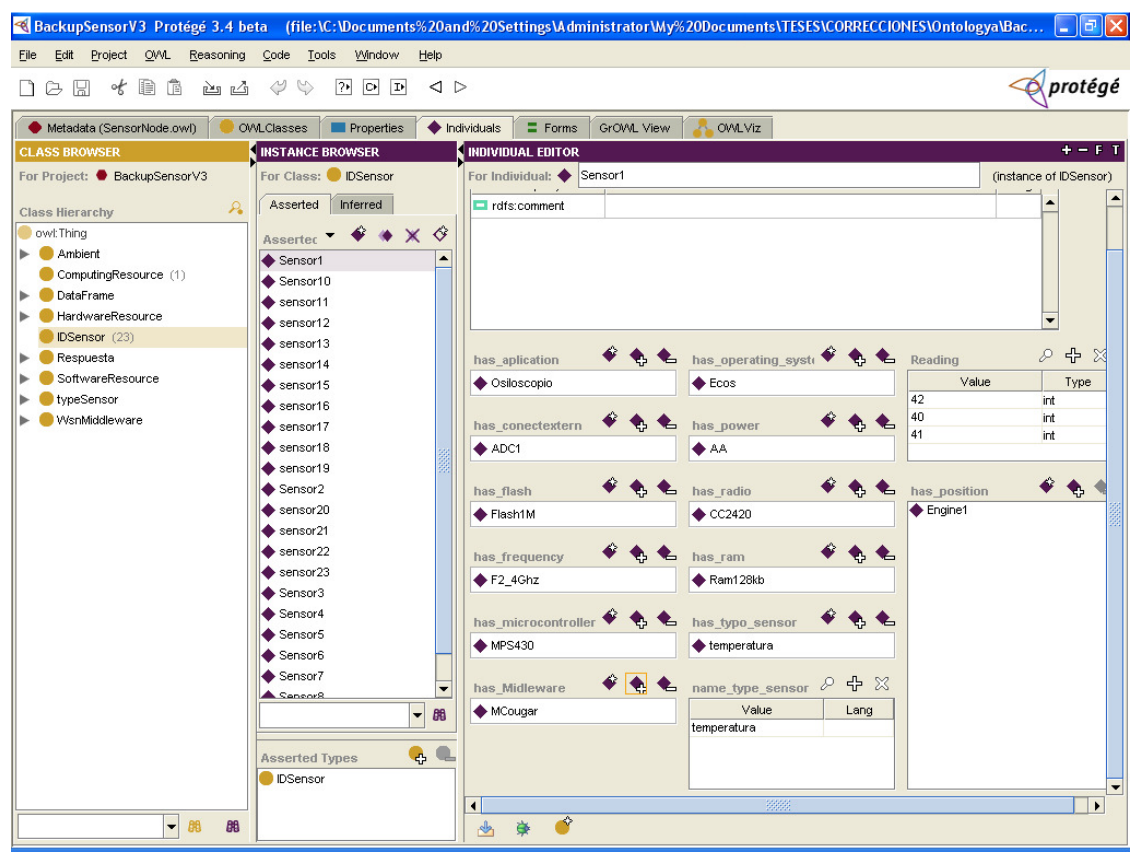

Figura 11 Interface gráfica da ferramenta Protégé-OWL

\section{WSN ontology User}

Os casos de uso mostrados na Figura 12, são responsabilidade do WSN ontology User (usuário da Rede). A seguir, cada caso de uso é descrito de forma resumida. 
- Consulta de recursos - Nesse caso de uso, o usuário, busca identificar os recursos de uma RSSF e seu potencial. O ator realiza uma consulta por diferentes parâmetros, tais como plataforma, heterogeneidade, etc.

- Consulta da aplicação - O usuário da RSSF busca identificar informação da rede e do ambiente. O usuário pode realizar essas consultas sobre a base de conhecimento para atender diferentes finalidades, como por exemplo, identificar estado dos nós sensores, dados de redes distintas, dados relacionados a execuções realizadas, entre outros.

- Execute Ação- Com a informação inferida o usuário pode executar alguma ação.

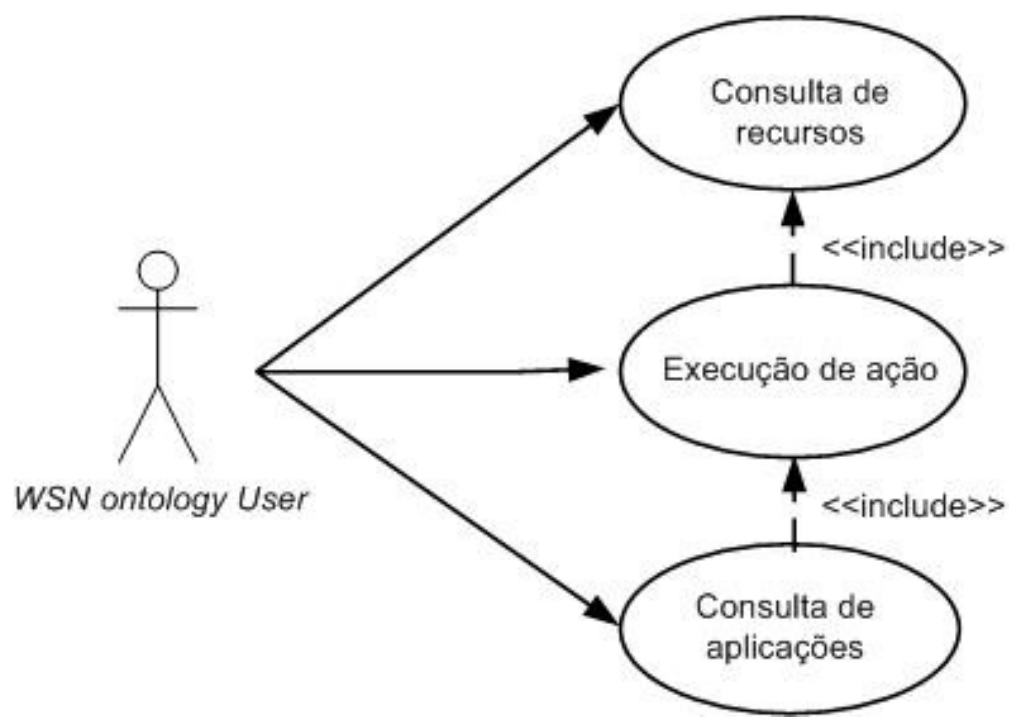

Figura 12 Casos de uso para o WSN ontology User

\section{Integration Component}

Este ator é um componente da arquitetura de software associado às ontologias e responsável pela realização de alguns casos de usos importantes incluídos nos diagramas de casos de uso dos atores humanos descritos acima. Esses casos de uso têm relação com tarefas de manutenção das ontologias e do uso das ontologias em diferentes cenários do ambiente. 


\subsection{Estrutura e conteúdo das ontologias}

É o resultado de uma abordagem exploratória que consistia em definir uma taxonomia inicial abrangente e investigar as possibilidades de utilização de mecanismos de inferência no domínio de RSSF. Iniciamos a definição das ontologias pela identificação dos elementos mais básicos presentes no domínio de RSSF, tais como recursos de hardware (por exemplo, CPU, memória), recursos de software (por exemplo, middleware, SO, ferramentas), dados de entrada e saída de aplicações, usuários, etc. Essa tarefa foi baseada em um trabalho de investigação preliminar e na discussão com especialistas no domínio.

Nessa etapa do desenvolvimento das ontologias, os conceitos relacionados à estrutura das RSSF adotados na ontologia foram inspirados pelo padrão arquitetural descrito em (RUSSOMANNO; KOTHARI; THOMAS, 2005). A reunião de conceitos importantes no formato de padrão arquitetural de RSSF facilitou a concepção da ontologia para a rede de sensores sem fio, mostrada na Figura 13. O padrão foi especialmente relevante na descrição de conceitos e relacionamentos comuns ao domínio de RSSF como um todo, promovendo o uso da ontologia como base para integração de diferentes sistemas.

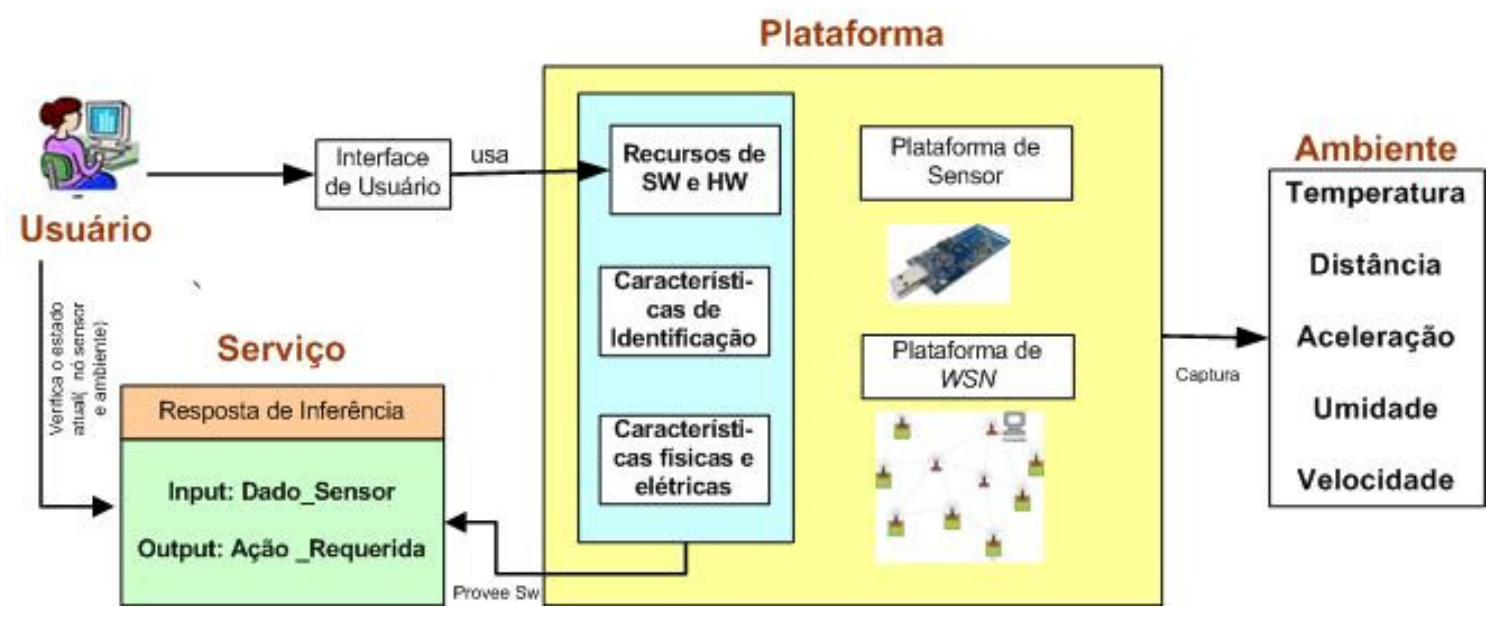

Figura 13 Concepção da ontologia para RSSF 


\subsubsection{Ontologia Geral}

$\mathrm{Na}$ Figura 13 mostra os quatro componentes principais do contexto da ontologia: o usuário, o serviço, o ambiente e a plataforma. O usuário interage com o sistema através de uma interface de usuário que se encontra no software da plataforma. A plataforma consiste em duas partes importantes; uma plataforma do sensor, onde se encontram todos os sensores e dispositivos, e uma plataforma da RSSF que suporta principalmente as características e condições atuais da rede; a plataforma dispõe os dados, sejam constantes ou variáveis de hardware ou software para que o motor de inferência na ontologia ofereça um resultado.

O refinamento posterior dos conceitos resultou em quatro ontologias principais da hierarquia de classes, mostrada na Figura 14.

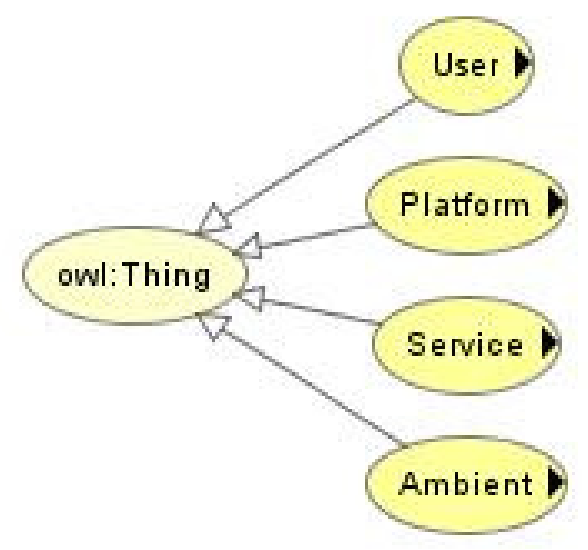

Figura 14 Ontologia Geral

Os seguintes sub-ramos das classes principais da hierarquia de classes são mostrados na Figura 15.

Todo o processo de desenvolvimento das ontologias foi realizado em etapas sucessivas e de modo incremental. A cada nova iteração, as ontologias existentes foram revistas e sofreram acréscimos, eliminações e outras alterações semânticas em sua estrutura. 


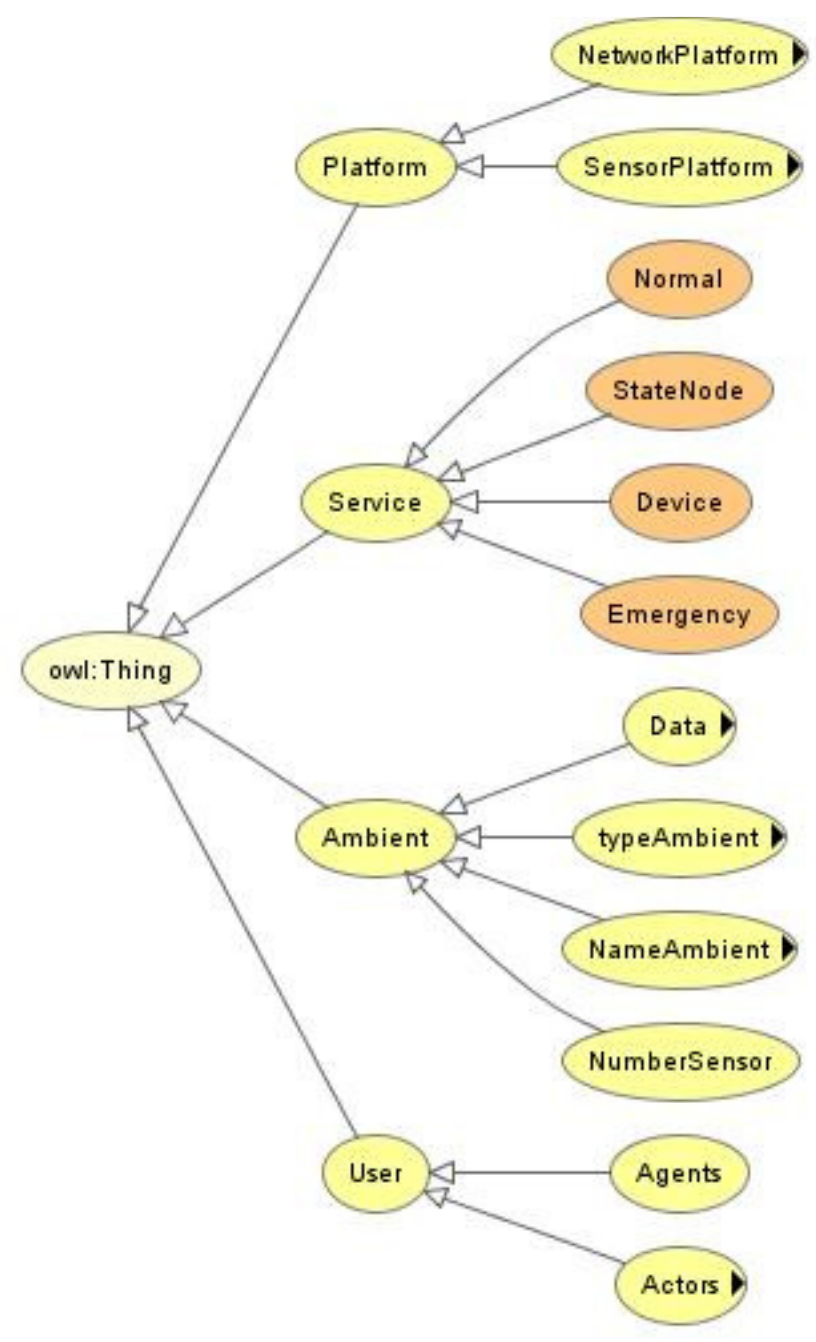

Figura 15 Sub-Classes da Ontologia Geral

A Figura 15 mostra o sub-ramo da ontologia relacionado aos conceitos comuns ao domínio de RSSF de um modo geral (Platform). Nesta fase de esboço da ontologia o sub-ramo define os conceitos, tais como, componentes de software, componentes de hardware do nó sensor (SensorPlatform) mostrado na Figura 16, e as característica da rede (NetworkPlatform). Os conceitos presentes neste sub-ramo da ontologia são fortemente relacionados aos conceitos do nó sensor apresentados no sub-ramo da Figura 16, os dois sub-ramos podem ser usados em conjunto como base para mapeamento entre RSSF distintas. 
A Classe relacionada ao conteúdo das respostas e dispositivos (Service) da aplicação, os conceitos definidos nessa classe dependem do tipo de aplicação ou ambiente e da classe (Platform).

O conteúdo da aplicação das RSSF (Ambient), os conceitos definidos nessa sub-classe descrevem 0 ambiente onde serão espalhados os nós sensores, 0 tamanho do ambiente, quantidade necessária de nós, entre outras. Finalmente o ator encarregado de monitoramento dos dados (User), os conceitos presentes nesta sub-classe descrevem as pessoas que precisarão da informação final.

O ramo da ontologia que trata das plataformas combina os ramos de plataforma do sensor e o ramo de plataforma da rede, com o objetivo de facilitar a classificação dos conceitos.

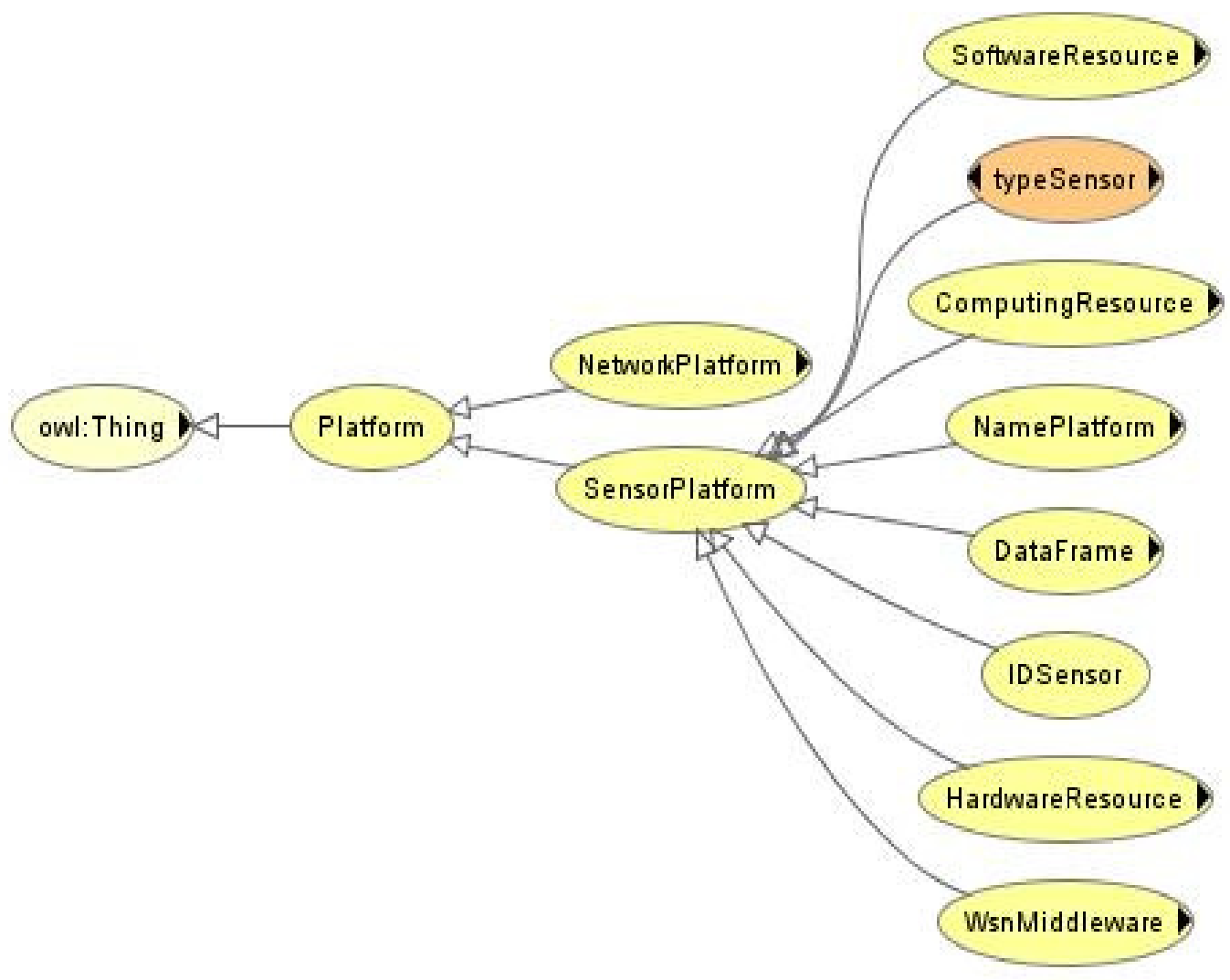

Figura 16 Ontologia da plataforma do sensor 


\subsubsection{Ontologia da Plataforma (Platform)-classe do nó sensor (SensorPlatform)}

A Figura 17 mostra a classe principal chamada SensorPlatform. Esta ontologia contém uma hierarquia de sub-classes e propriedades relacionadas ao domínio de plataformas do nó sensor. Isso inclui, entre outros, conceitos como, tipo de sensor, middleware, frame de dados, componentes de hardware, componentes de software, recursos de funcionamento. As classes definidas nessa ontologia serão reusadas como atributos para qualquer aplicação. Esta ontologia, como as demais do conjunto proposto, não pretende ser exaustiva, uma vez que pode ser estendida a qualquer tempo para inclusão de novas classes representando novos conceitos. Esta ontologia descreve o seguinte tipo de informação:

A Classe da ontologia que trata do tipo de sensor define o tipo de medida ou sinal físico capturado (temperatura, velocidade, aceleração, etc).

O sub-ramo relacionado ao conteúdo de frame de dados (DataFrame). Os conceitos definidos nesse sub-ramo descrevem organização, formato e tamanho de conjuntos de dados armazenados pelos nós. O frame de dados depende do tipo de aplicativo instalado para a captura destes, mas basicamente, o frame contém dados tais como, dest addr, handlerlD grouplD, msg len, source addr counter, channel, readings. Este dados capturados pelos sensores são também chamados de dados dinâmicos (TINYOS, 2003).

Os dados são usados principalmente como entrada e/ou saída na execução de aplicações na RSSF. A descrição dos dados com base nesses conceitos pode ser usada, por exemplo, como parâmetro na escolha de recursos para armazenamento, promovendo a alocação eficiente desses recursos na rede de sensores sem fio. Outro caso comum de uso de metadados sobre dados é a identificação de compatibilidade de formato de dados de entrada e os requisitos definidos por uma versão específica de uma aplicação. Metadados específicos sobre o domínio de origem e utilização dos dados podem ser acrescidos aos conceitos genéricos definidos inicialmente. 


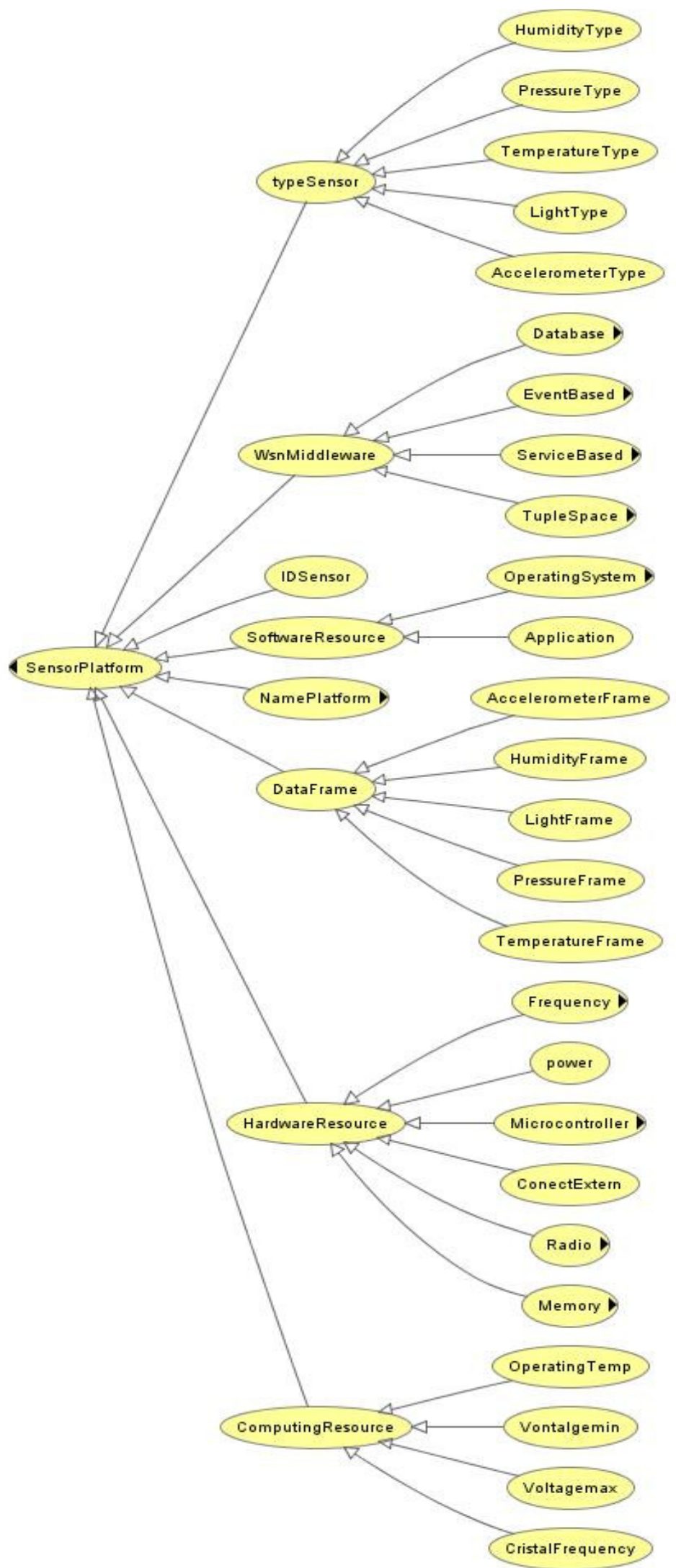

Figura 17 Classe Geral da Plataforma do sensor 
A Figura 18 mostra a classe da ontologia que trata do middleware para RSSF, define sub-classes para descrever o tipo de middleware. Segundo Henricksen e Robinson (2006) classificam-se em middleware baseados em: banco de dados, eventos, tuples Space e serviços. Cada classificação é composta por um conjunto de middleware que existe para as RSSF, e cada middleware depende do tipo de sistema operacional do nó sensor.

O ramo da ontologia que trata de recursos de software define o tipo de aplicativo instalado para a captura dos dados, estas classes estão relacionadas à classe de sistema operacional e à plataforma de sensor usada. A maioria dos aplicativos estão desenvoltos em NesC e usam o Cygwin como visualizador. Figura 18 mostra o ramo da ontologia que trata de sistemas operacionais; esta classe está relacionada com a classe NamePlatform que descreve as plataformas dos sensores, com as quais os sistemas operacionais são compatíveis com este sub-ramo organizado por produto. O Sistema Operacional mais conhecido e usado pelas diferentes plataformas existentes para RSSF é o TinyOS.

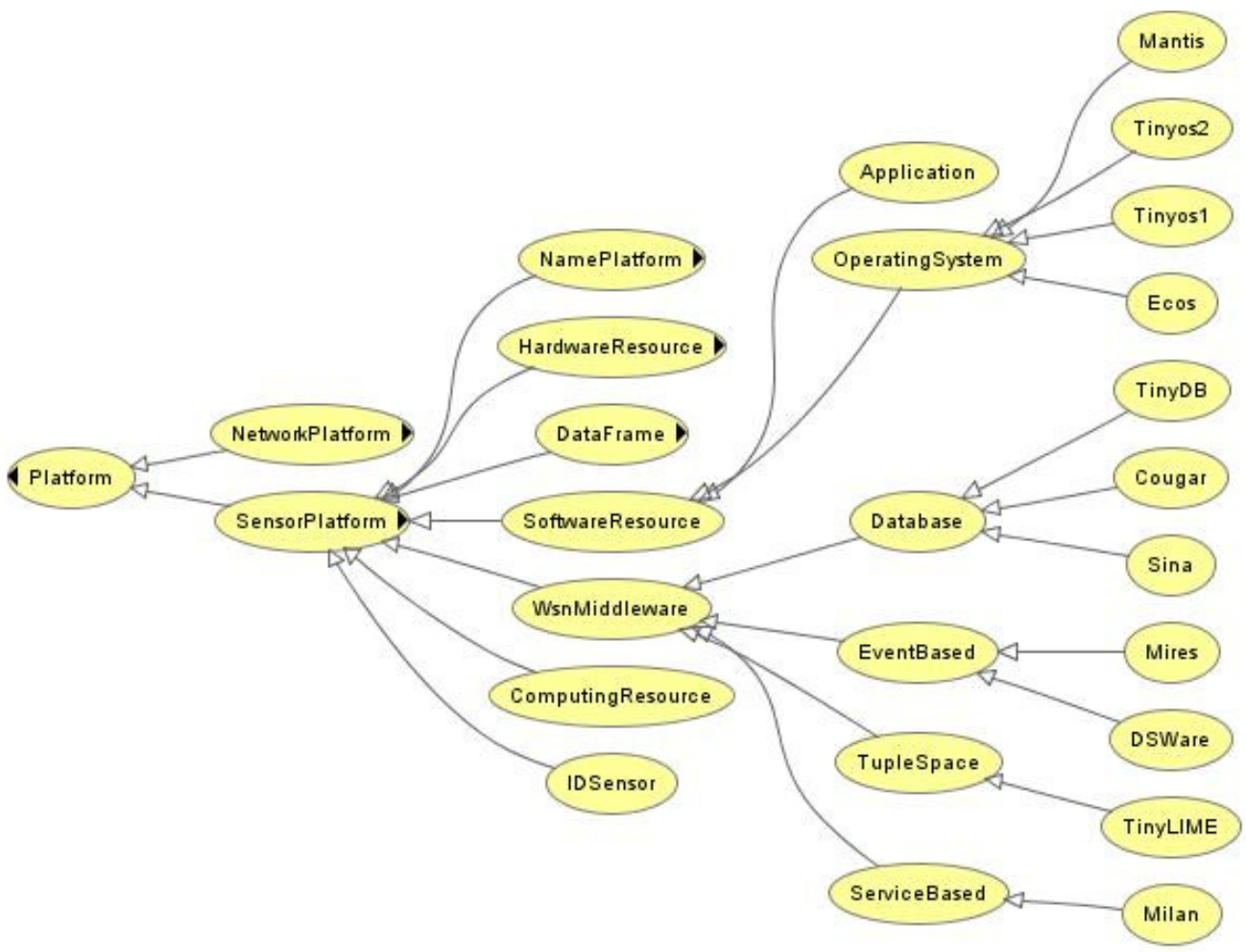

Figura 18 Classe WsnMiddleware - Ontologia Platform 


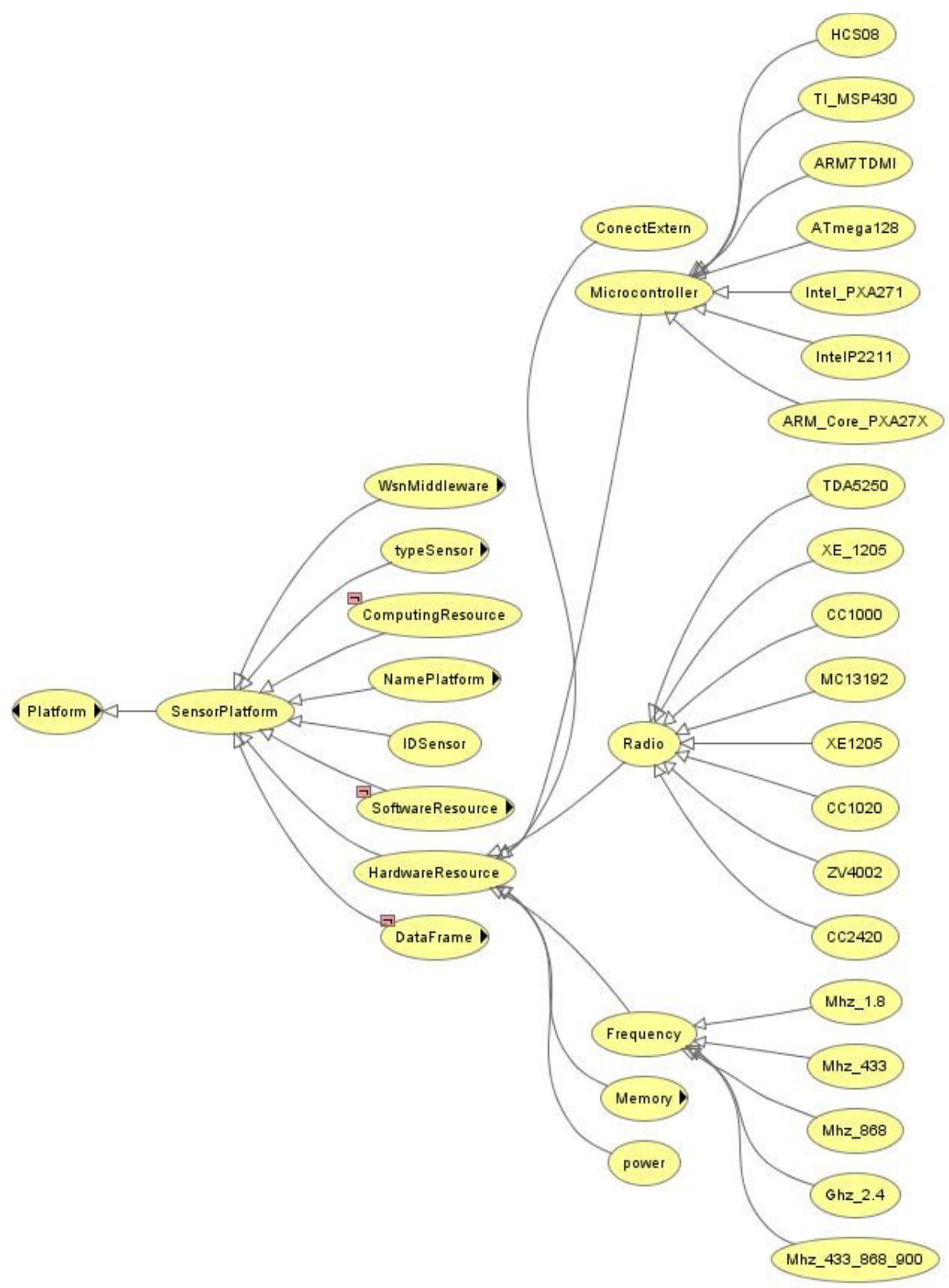

Figura 19 Classe Hardware Resource - Ontologia Platform

O ramo da ontologia que trata de recursos de hardware define sub-ramos e suas respectivas propriedades, descreve suas características e define conceitos tais como processador, memórias, fonte de poder, os conectores externos, antena, entre outros (MOTEIV, 2007), como ilustra-se na Figura 19.

O sub ramo relacionado ao conteúdo dos recursos de funcionamento (ComputingResource) é a classe que contém os dados necessários para um bom 
funcionamento dos nós, porém está dividido em sub-classes como voltagem máxima, voltagem mínima, freqüência do cristal e temperatura (MOTEIV, 2007).

Finalmente, a Classe (IDsensor), não contém sub-classe mas está composta por propriedades object e datatype, que estão completamente relacionadas ao conteúdo de cada nó a ser controlado. Por exemplo, temos a propriedade hás_IDsensor que é do tipo funcional, que indica a relação com somente um sensor, a propriedade ha-s_operating_system cuja inversa pertence à classe OperatingSystem, porem esta classe é dependente de todas as classes nomeadas anteriormente, algumas destas propriedades estão ilustradas na Figura 20.

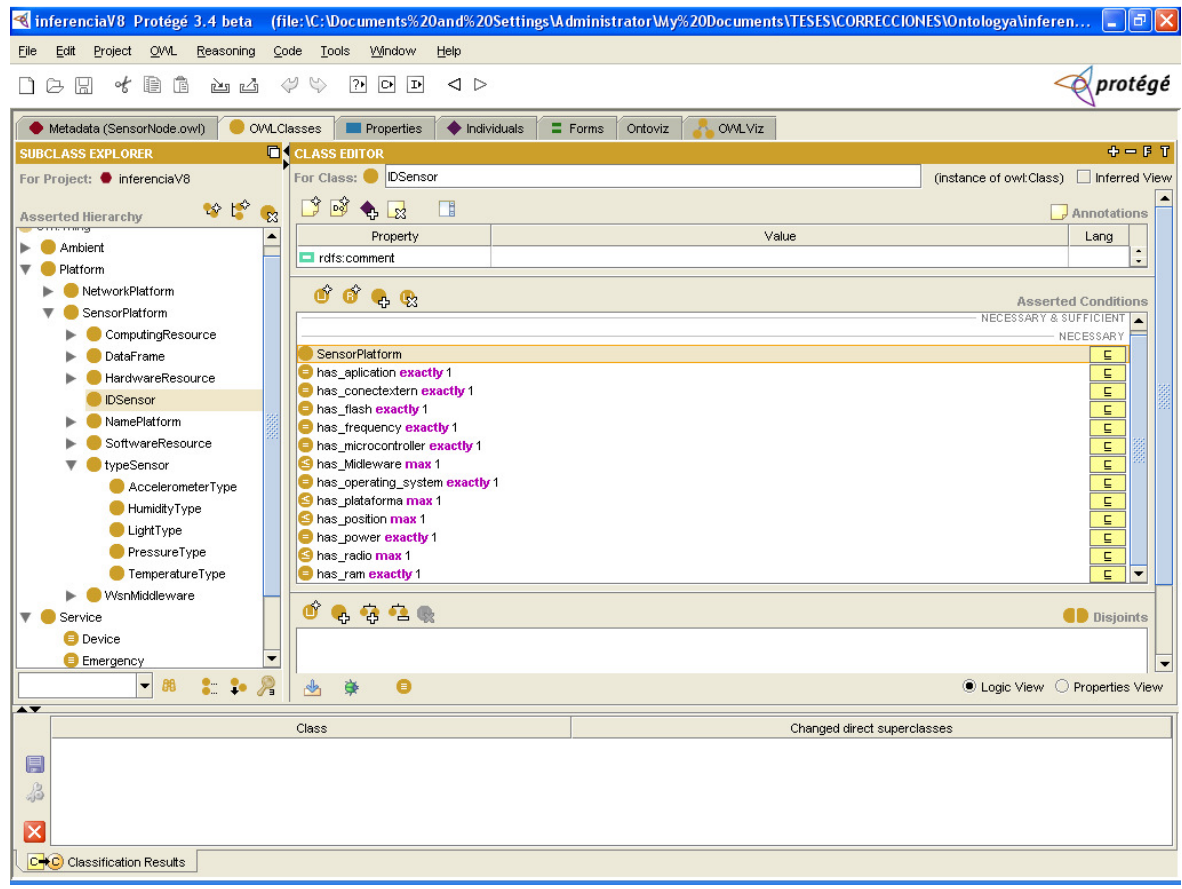

Figura 20 Restrições das propriedades da classe IDSensor

\subsubsection{Ontologia da Plataforma (Platform)- classe da rede (NetworkPlatform)}

NetworkPlatform é outra classe principal definida na Ontologia Platform inclui conceitos relacionados à recursos da rede, tais como topologia, protocolos de comunicação e conceitos de um sistema de comunicação (anotados respectivamente como: Topology, ComunicationSys, ComunicationProtocol baseados em (JURDAK; VIDEIRA; BALDIY, 2004). Por sua vez, estes conceitos 
também podem ser estendidos, por exemplo, pelo ator WSN ontology manager para descrever uma estrutura de rede de sensores sem fio concreto. Um estudo exaustivo desta ontologia poderia ajudar na seleção de protocolos de disseminação de dados em RSSF (PIRMEZ et al., 2006).

A Figura 21 mostra os sub-ramos que descreve esta ontologia; este sub-ramo é fortemente relacionado aos componentes da ontologia SensorPlatform apresentados na Figura 17. As duas ontologias podem ser usadas em conjunto como base para outras aplicações.

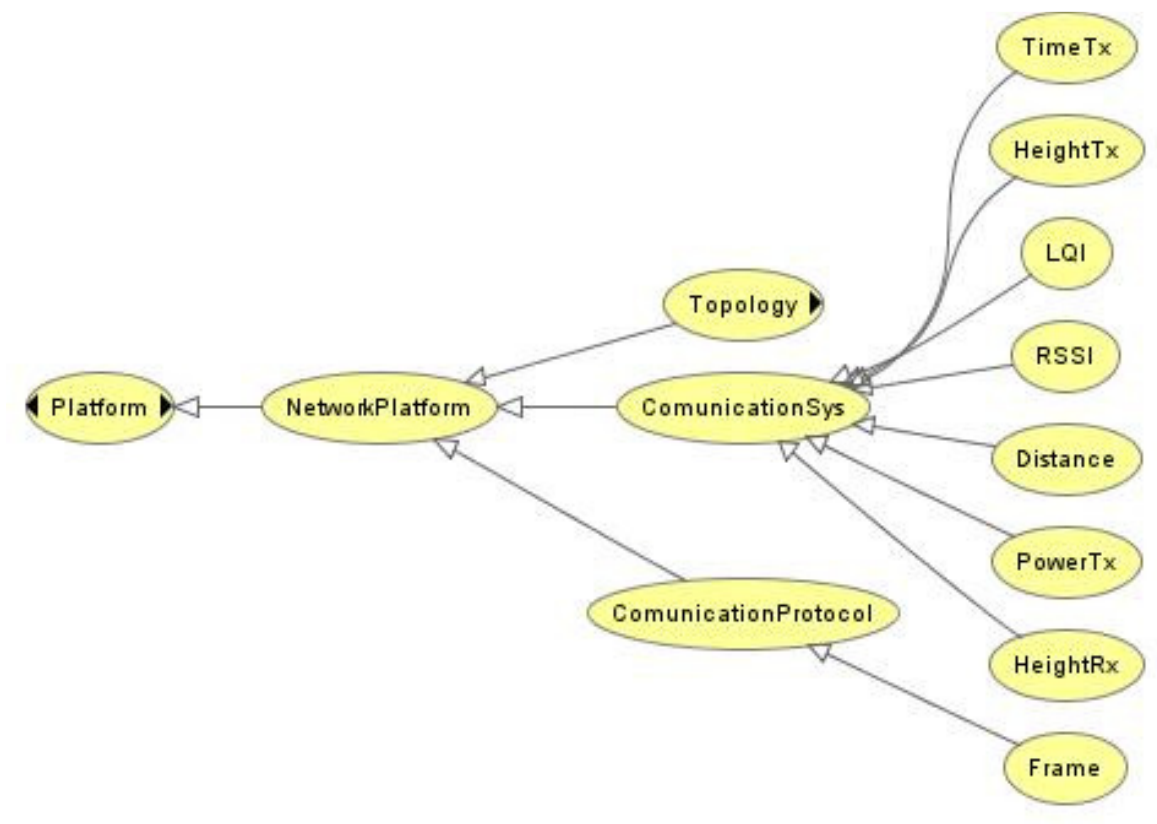

Figura 21 Classe da Plataforma da rede (NetworkPlatform)

\subsubsection{Ontologia do ambiente (Ambient)}

Em nossa base de ontologias, a ontologia do ambiente (Ambient) contém conceitos, propriedades e axiomas que são comuns a aplicações onde podem ser monitoradas ou controladas pela RSSF. A Figura 22 mostra a hierarquia de classes da Ontologia do Ambiente definida pelo WSN User Ontology. Essa hierarquia é baseada em quatro conceitos chave: número de sensores espalhados no ambiente, nome do ambiente, tipo de ambiente e os dados que aplicação precisa (anotados respectivamente como: NumberSensor, NameAmbient, typeAmbient, Data). 
Os dois principais ramos da hierarquia do tipo de ambiente (typeAmbient) são: lugares fechados e lugares aberto ou de acesso remoto (Indoor, Oudoor respectivamente).

Inclui-se um conjunto de conceitos relacionados especificamente, ao ambiente e às características para as quais se use a RSSF nesse ambiente, tais como casa, automóveis, escritórios inteligentes, florestas, oceano ou pontes. Tais conceitos têm um nível suficientemente alto de abstração para serem estendidos, por exemplo, pelo ator WSN user ontology, e descreverem os conceitos de seu próprio domínio de desenvolvimento, seus problemas e abordagens, a partir de uma terminologia comum. A ontologia Ambient deveria importar qualquer ontologia auxiliar e assim reusar os conceitos e propriedades da plataforma.

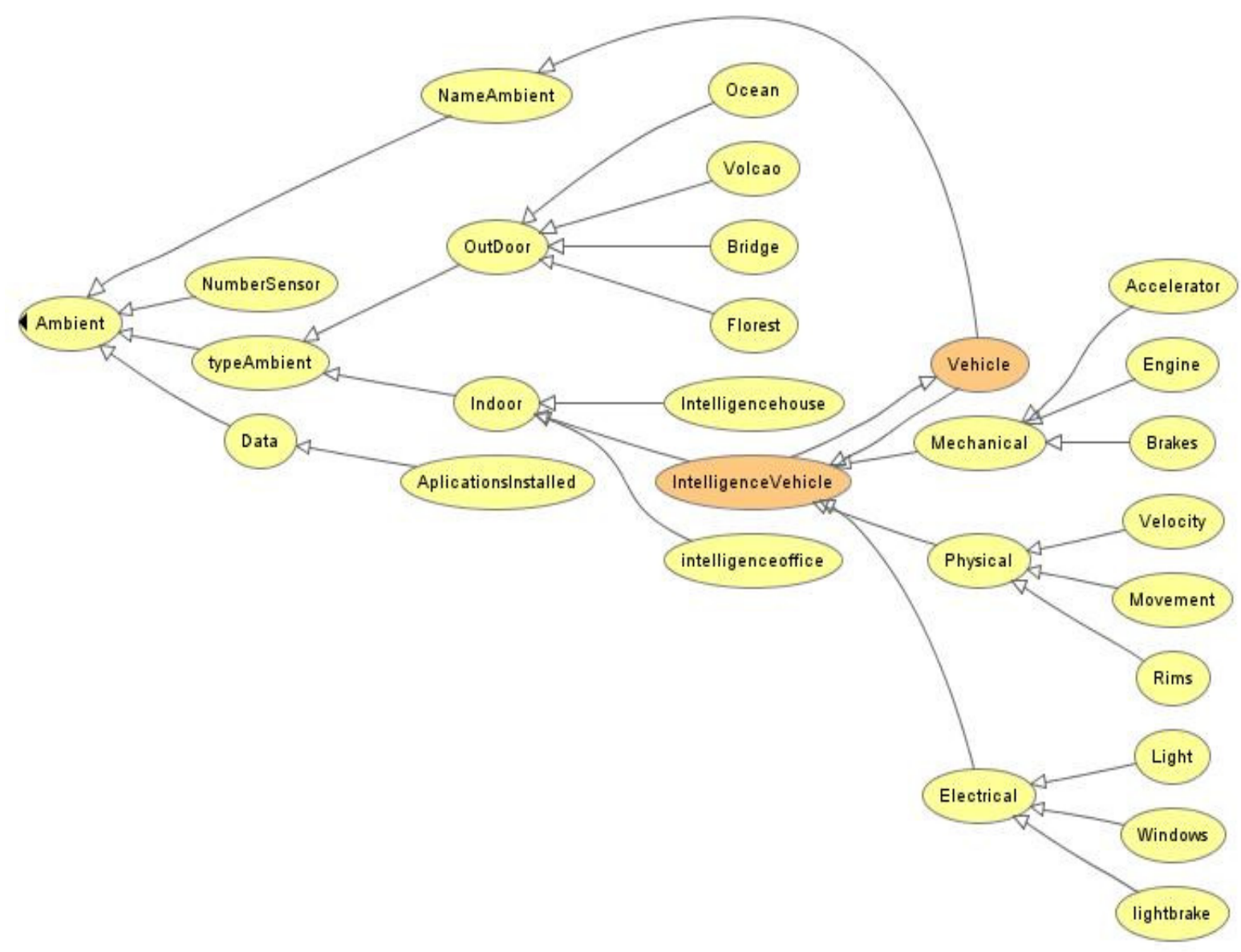

Figura 22 Ontologia do Ambiente 


\subsubsection{Ontologia de serviços (Service)}

O sub-ramo Service mostrado na Figura 23, de acordo com o tipo de aplicação, envolve conceitos que podem ser particularmente úteis para estabelecer uma conexão semântica para ação requerida.

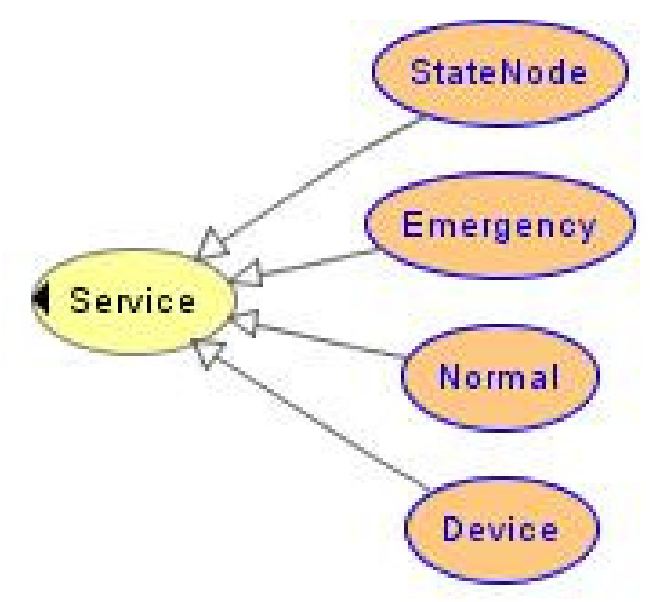

Figura 23 Ontologia de serviço

Esta Classe está relacionada à aplicação onde é utilizada a RSSF, ou seja, é uma ontologia dependente do domínio de aplicação e foi inspirada no padrão de ontologia descrito em (ULIERU; MADANI, 2006), esta ontologia também depende muito dos dados estáticos e dinâmicos dos nós, tanto para estado do ambiente como para o estado do nó.

A classe (StateNode), está composta por propriedades e restrições que permitem inferir o estado do nó sensor. A classe emergência (Emergency) é uma sub-classe da ontologia serviço e descreve o estado do ambiente, também composto por propriedades e restrições ou regras para inferir o estado normal ou Emergência.

A descrição das propriedades dos dispositivos é dada pela classe Device, ela descreve o dispositivo que está relacionado ao nó sensor, o estado e as operações do dispositivo. Os dispositivos são os atuadores que interagem com o ambiente. 


\subsubsection{Ontologia de usuário (User)}

Finalmente, a ontologia User; esta ontologia contém conceitos dos atores ou usuários das RSSF e dos agentes de software caso sejam implementados. Anotados respectivamente como: (AgentSoftware, actors) esta ontologia permite descrever as preferências do usuário. Um serviço especializado permite que o ambiente forneça os serviços que a usuário deseja.

Diferentes ontologias, semanticamente relacionadas, podem se conectar através de diferentes mecanismos. O mecanismo import, definido na linguagem OWL, permite a uma ontologia "importar" uma ou mais ontologias. Este mecanismo possibilita, à ontologia que importou referenciar e estender todos os conceitos e propriedades da ontologia importada, melhorando as características de compartilhamento e reuso do conjunto de ontologias. Conceitos e propriedades da ontologia importada recebem um prefixo referente ao espaço de nomes relacionados.

Outros conhecidos mecanismos voltados para o estabelecimento de relacionamento concreto de ontologias são os chamados mecanismos de mapeamento (mapping) e e-connection. Esses mecanismos não são explicitamente tratados no presente trabalho. Todos os mecanismos apresentados constituem alternativas relevantes para promover escalabilidade na base de ontologias.

Contudo, a estrutura da ontologia tornou-se excessivamente ampla tanto em relação à variedade, quanto em relação à quantidade, potencialmente crescente, de diferentes conceitos, propriedades e axiomas. Assim, com o objetivo de promover facilidades de manutenção e escalabilidade, separamos a ontologia original em um conjunto de ontologias conectadas. 


\section{Capítulo 6}

\section{Estudo de Caso}

Neste trabalho foi proposta uma abordagem flexível e extensível para a realização de controle de dados de uma RSSF baseado em ontologia.

Diferentemente dos serviços para o controle e o gerenciamento de dados tradicionais que descrevem dados ao usuário final, baseados em middleware que permitem fazer o controle dos dados de uma forma, mais simples; as ontologias (por exemplo, descrições semânticas de modelos do domínio) são criadas para descrever declarativamente informações da rede e do estado atual da aplicação utilizando uma linguagem de ontologia expressiva.

Para avaliar a proposta do controle e gerenciamento de dados das RSSF baseado em semântica foram realizadas algumas consultas, tendo como parâmetros as características dos recursos computacionais e a informação coletada pelos nós sensores. No experimento foram incluídas consultas simples (ou seja, apenas uma característica ou informação do sensor) e consultas múltiplas (ou seja, mais de uma característica).

\subsection{Ambiente de Teste}

O Objetivo do estudo de caso é realizar o gerenciamento de dados de uma rede de sensores sem fio, distribuída em um ambiente; neste caso em particular foi um ambiente veicular, baseado no trabalho de (FLORIDO, 2008) para a verificação do estado do veículo e o estado do nó sensor.

Este ambiente foi escolhido porque os automóveis inteligentes constituem uma área ativa de pesquisa, já que continua crescendo o interesse das indústrias automotivas em fabricar veículos cada vez mais modernos aproveitando o rápido 
desenvolvimento da tecnologia da informação e os serviços da telemática (combinação da telecomunicação e informática). Em (LI et al., 2005), são descritos serviços que requerem automóveis inteligentes, sendo eles: navegação, assistência em emergências, segurança, diagnóstico remoto e entretenimento.

O estudo de caso permite esclarecer os conceitos e técnicas para desenvolvimento do sistema, para o qual serão criadas novas ontologias, nas quais serão considerados os aspectos chaves da informação de contexto. O Ambiente deste estudo de caso é mostrado na Figura 24; deve-se levar em conta, no posicionamento e na quantidade de nós sensores a serem instalados, as características próprias da rede a ser implementada (topologia, tecnologia de comunicação, etc.) e os tipos de fenômenos a serem avaliados.

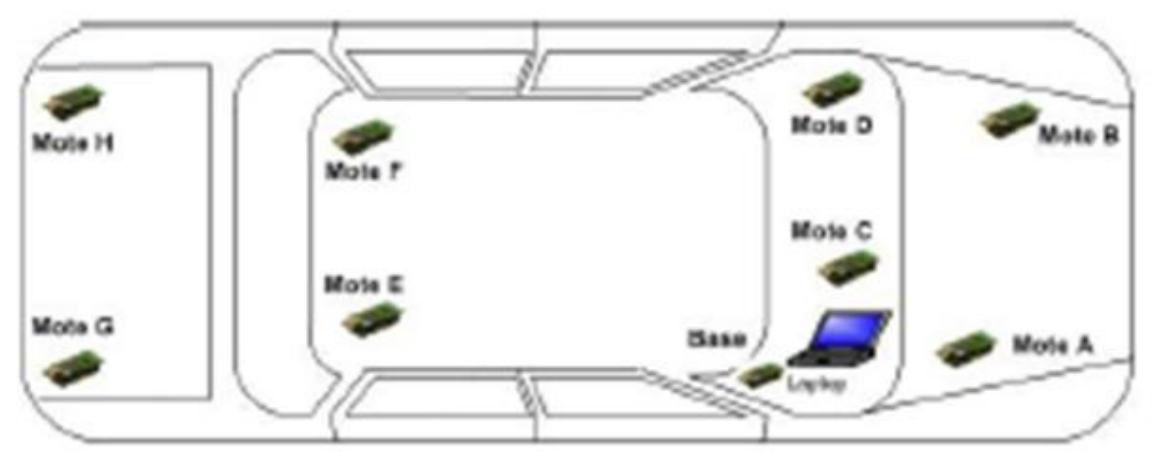

Figura 24 Visualização do ambiente de uma Rede de Sensores Sem Fio (FLORIDO, 2008)

\subsubsection{Instalação do Ambiente}

Para que o ambiente da rede pudesse funcionar, foram necessárias a instalação e configuração dos nós sensores. Uma descrição detalhada dos dispositivos TmoteSky utilizados, do sistema operacional e da aplicação pode ser encontrada no Anexo B. Resumidamente, foi feita a instalação da ferramenta MSRsense, esta ferramenta usa o aplicativo Osciloscope para a coleta dos dado e funciona sobre o sistema Operacional TinyOS. A quantidade e tipo de dados coletados dependem das configurações da aplicação. 


\subsubsection{Ferramentas de apoio}

- Para a criação das ontologias utiliza-se o Protégé 4.0 o qual permite a utilização de plugins para a visualização das ontologias como OWLviz usada no capitulo 5 ;

- Ferramenta de apoio à inferência baseado no mecanismo de inferência Pellet, também apresentado no Capítulo 3;

- A Interface para consulta implementada sobre a API Protégé-OWL, e utilizando a linguagem de consulta SPARQL. A linguagem de consulta SPARQL foi comentada no Capítulo 3.

\subsection{Seleção Direta e Semântica}

A avaliação do modelo proposto vai ser centrada unicamente no cenário de busca de dados dos sensores e do ambiente do estudo de caso. Queremos mostrar que a abordagem semântica consegue resultados eficientes, em situações práticas de recuperação e uso de metadados na RSSF. Esta será comparada com abordagens de gerenciamento de metadados convencionais, nas quais as comparações são feitas sobre os valores explícitos dos atributos descritos na base dos metadados. Em tais situações as informações disponíveis são comumente incompletas ou implícitas.

As consultas foram realizadas no Repositório Semântico, especificando-se consultas simples ou múltiplas com seleção direta e semântica. Considerando que existem dois tipos de dados estáticos e dinâmicos, a origem dos dados estáticos tipicamente são as bases de dados que contêm registros de informação do sensor como, sistema operacional, tipo de aplicação, tipo de leitura a medir, ou informação do ambiente para o estudo de caso, como o registro do veículo (ano, cor, marca modelo, etc). Os dados dinâmicos podem incluir leituras do sensor, a informação em tempo real do veículo, assim como a informação dos próprios nós sensores. Com a ajuda do uso da semântica, pode-se ter um melhor processamento das séries de dados estáticos e dinâmicos disponíveis. 
Entende-se por consulta simples quando o usuário tem necessidade por apenas um dos dados, sejam estáticos ou dinâmicos, por exemplo, Id e as leituras dos nós ou os nós que contêm sistema operacional TinyOS (considerando que o usuário desejará fazer a instalação de uma aplicação que só pode ser executado no TinyOS).

Por outro lado, consultas múltiplas referem-se à necessidade de mais de uma característica do recurso, por exemplo, deseja-se instalar uma aplicação que demande o sistema operacional TinyOS e que seja do tipo de hardware TmoteSky ou conhecer os valores dos sensores localizados no motor do veículo e que os dados capturados sejam de temperatura.

A caracterização destas informações pode ser relevante para múltiplos casos, como por exemplo, para o processo de manutenção e conserto preventivo dos sensores e ambientes que os sensores monitoram.

\subsubsection{Teste 1}

\subsubsection{Seleção Direta}

Neste teste fazemos uma consulta onde obtemos informação do ambiente; serão efetuadas consultas como: que sensor, que características, onde este localizado, que tipo do sinal captura e estado atual do motor.

Na seleção direta utilizamos a ferramenta MSRsense (CHEONG et al., 2005) descrita no capitulo 4, que utiliza a SQL Server Management Studio Express para o armazenamento centralizado e recuperação de informação dos dados da RSSF. O fato de ser um sistema de recuperação de informação tradicional permite armazenar, exportar, importar, migrar e recuperar os dados de forma classificada e segmentada, atendendo à necessidade de cada usuário final, mas não permite a inferência automática de novas informações. Uma descrição detalhada da instalação desta ferramenta é encontrada no anexo $\mathrm{C}$. 
A maioria das linguagens de processamento de consulta é baseada na sintaxe SQL-like. Por exemplo, no projeto de TinyDB (MADDEN et al., 2005) desenvolvido na Universidade de Berkeley, a linguagem de consulta consiste de sentenças SELECT-FROM-WHERE-GROUPBY-HAVING para suportar a seleção, projeção, agregação, e agrupamento (GEHRKE; MADDEN, 2004). Esta linguagem é eficiente para mecanismos de armazenamento, orientada a base de dados. Se o usuário realiza uma consulta tal como SELECT data FROM base de dados WHERE data $=$ "11-20-05" e temperatura $=40$, a pergunta retornará os resultados previstos, mas utiliza simples comparações de string e integer. Esta abordagem ignora todos os relacionamentos entre as duas partes de dados. Por exemplo, a data pode ser relacionada à temperatura por um relacionamento do has_temp, permitindo conexões do nó a nó. A interface de tinyDB é ilustrada na Figura 25, mais informações e sua instalação desta ferramenta encontram-se no Anexo C.

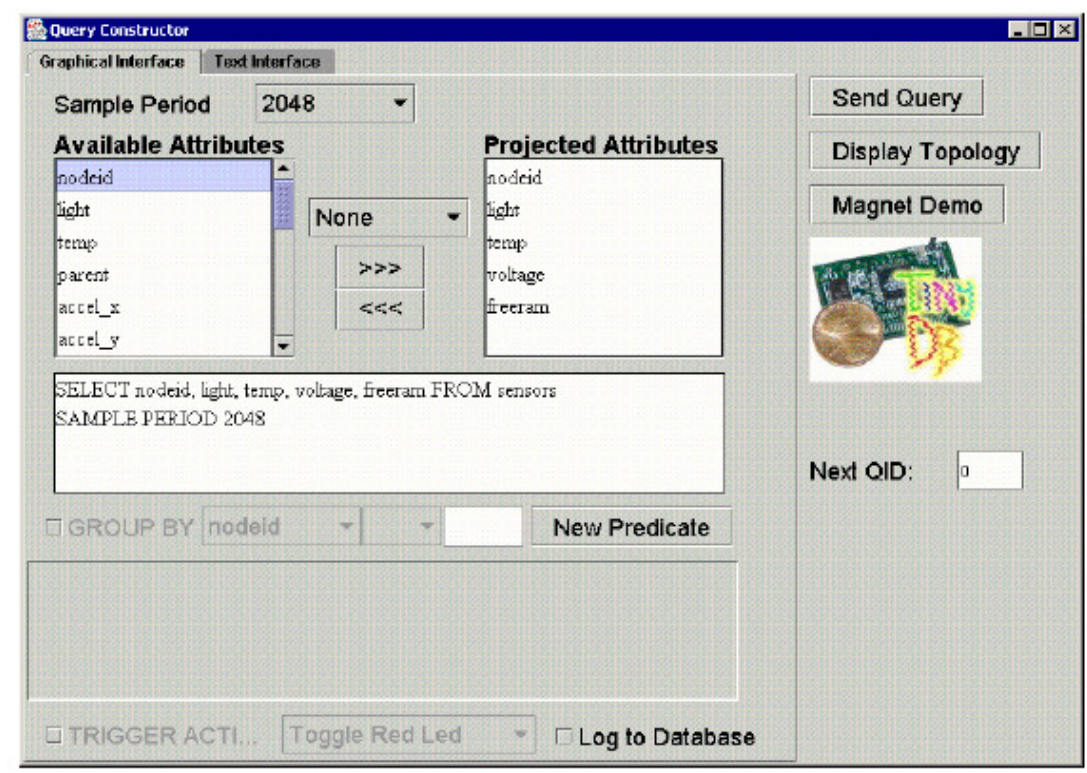

Figura 25 Tela do construtor de queries TinyDBMain (MADDEN et al. 2005)

\subsubsection{Seleção Semântica}

Além de atuar como taxonomia, um conjunto de ontologias deve funcionar, principalmente, como base para recuperação de informação na RSSF. Através do uso de linguagens de consulta e de mecanismos de inferência sobre a base de 
conhecimento, o usuário da rede de sensores pode propor diferentes questões como as definidas no Capítulo 5 (seção 5.1.3).

A informação dos sensores pode ser procurada por muitas razões, a informação significativa é transformada em conhecimento sobre o fenômeno de interesse, a informação observada de um ou mais nós é utilizada para identificar a presença do fenômeno de interesse, os nós vizinhos podem ser usados para a verificação do fenômeno e detectar a localização exata.

Com a seleção semântica, conseguimos ter os dados melhor relacionados. $O$ Quadro 2 mostra o exemplo de código SPARQL para a primeira consulta e o resultado é mostrado na Figura 26. Uma vantagem de se usar ontologia é a possibilidade de inferir nova informação (estado atual do motor) mostrada na Figura 29.

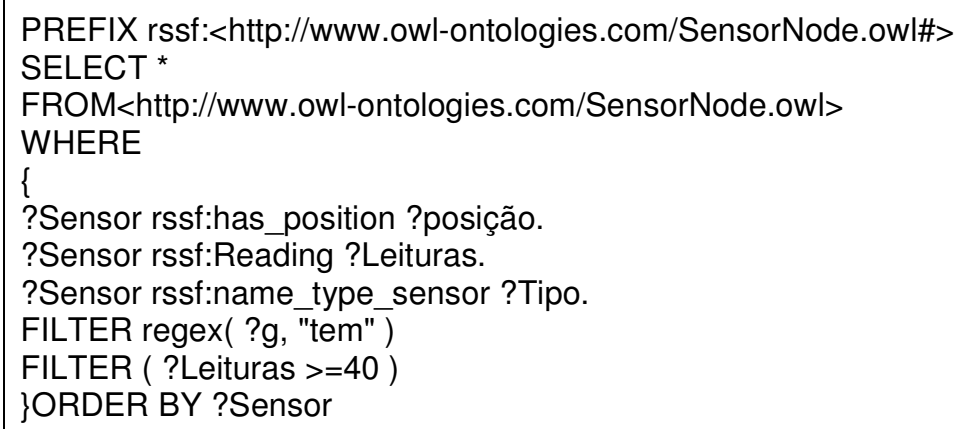

Quadro 2 Código SPARQL para localização e leituras dos nós

\begin{tabular}{|c|c|c|c|c|}
\hline \multicolumn{5}{|l|}{ Results } \\
\hline Sensor & Posiçẫo & Leituras & Tipo & \\
\hline Sensor2 & & 40 & temperatura & 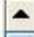 \\
\hline Sensor2 & & 42 & temperatura & \\
\hline Sensor1 & & 40 & temperatura & \\
\hline Sensor1 & & 41 & temperatura & \\
\hline Sensor1 & & 42 & temperatura & \\
\hline Sensor3 & & 42 & temperatura & 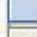 \\
\hline Sensor3 & & 45 & temperatura & $\nabla$ \\
\hline
\end{tabular}

Figura 26 Resultado da consulta do teste -1

Um atrativo pelo modelo semântico é a possibilidade de inferir-se conhecimento, apenas pela prévia definição de axiomas e de informações 
parcialmente completas. Analisemos, por exemplo, a realização de subsumption pelo mecanismo de inferência sobre a ontologia de Serviço (Service ontology).

- A classe Emergency mostrada na Figura 27, é descrita como sub-classe da classe Service. Relaciona-se com a classe IDSensor, através das propriedades Object, como: has_position, has_type_sensor e a propriedade Datatype como: Reading.

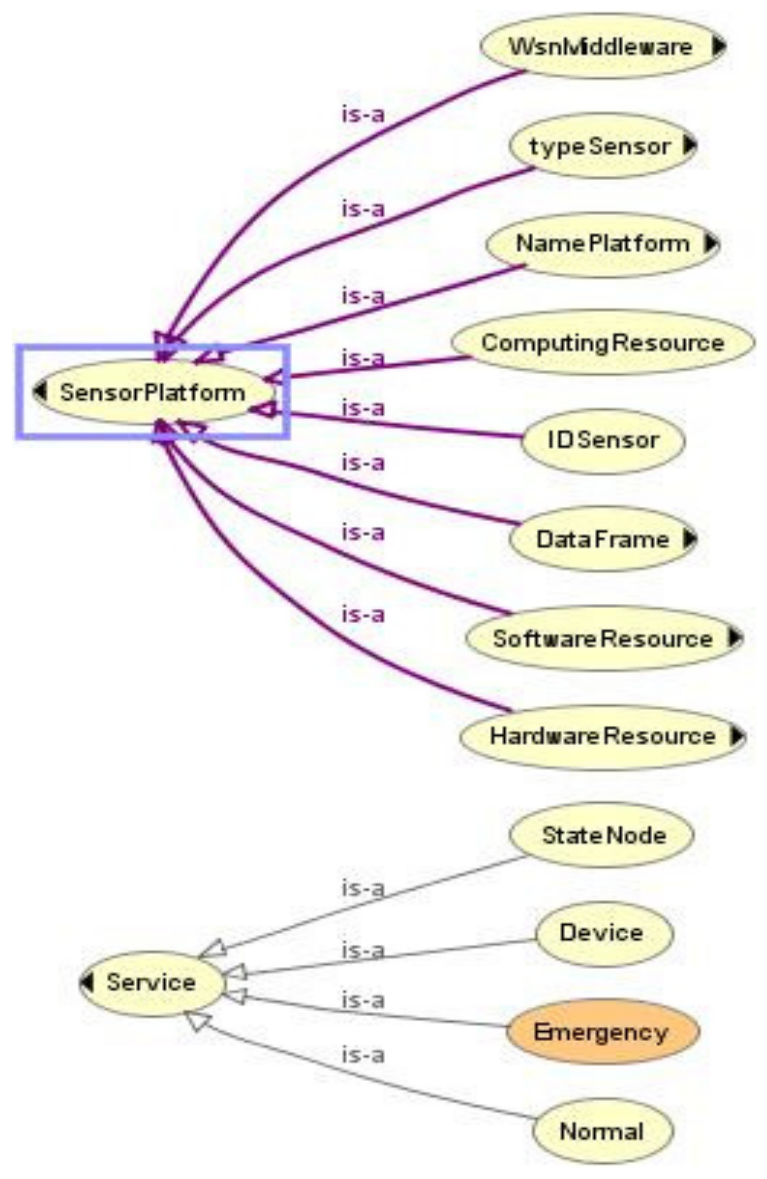

Figura 27 Ontologia de Serviço

Depois que a tarefa de subsumption é realizada sobre a hierarquia pelo mecanismo de inferência, uma nova estrutura hierárquica é produzida e a classe Emergency é classificada também como sub-classe da classe IDsensor, como ilustra a Figura 28. 


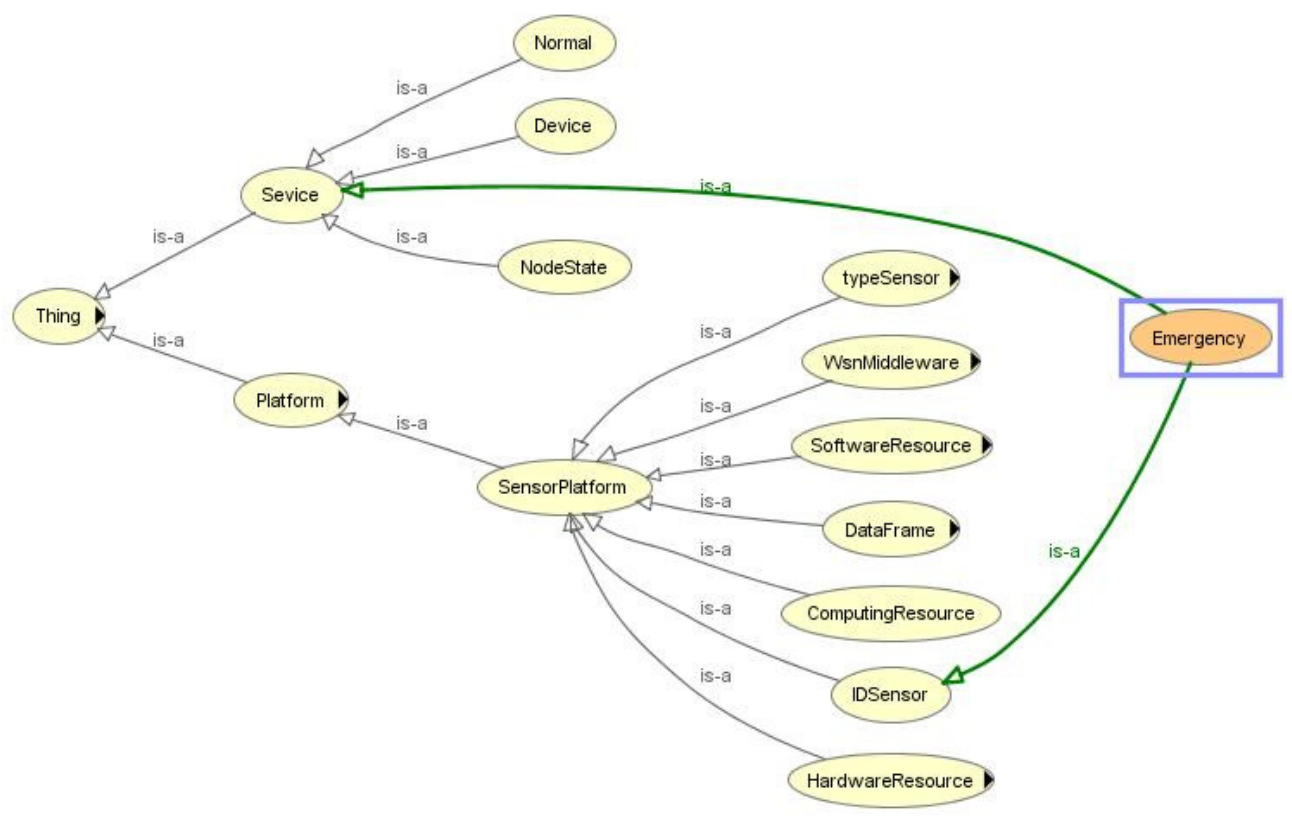

Figura 28 Ontologia Inferida

De acordo com (RECTOR et al., 2004), classes definidas são "classes para as quais existe pelo menos um conjunto de condições necessárias e suficientes". Em outras palavras, isso significa em nosso exemplo, que qualquer indivíduo da classe IDsensor que satisfaça a condição de definição, será classificado como um indivíduo da classe Emergency. A Figura 29 apresenta a informação inferida da ontologia.

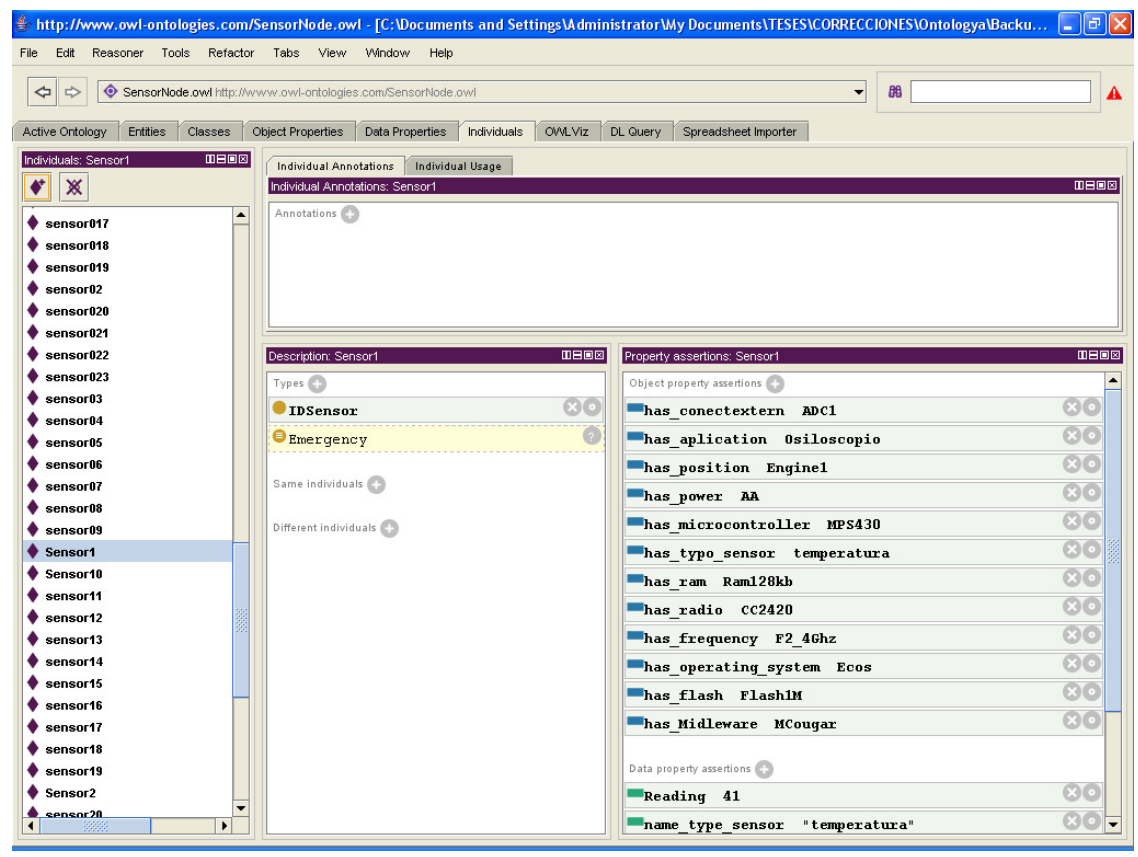

Figura 29 Janela do dado inferido do teste 1 utilizando Pellet 


\subsubsection{Teste 2}

Serão efetuadas aferições sobre a eficácia dos resultados no sentido de incrementar a disponibilização da maior quantidade possível de informação dos nós sensores, por exemplo:

- Para execução de aplicações, OscilloscopeRF, Surge;

- Para verificação dos nós com um sinal baixo, por exemplo, fatores de sinal Received Signal Strength Intensity (RSSI) na camada de comunicação;

- Para consulta de temperatura, por exemplo, na camada de aplicação monitora-se a temperatura para determinadas localizações e peças do veículo. Esta consulta pode ser realizada através de caminhos distintos: temperatura ou TEMPERATURA; os dois caminhos produzem ao cabo da extração semântica de informações uma quantidade igual de dados.

A Figura 30 mostra os conceitos definidos no conjunto de ontologias descritas no Capítulo 5, como classes ou indivíduos.

\begin{tabular}{|c|c|c|c|c|c|c|}
\hline \multicolumn{7}{|l|}{ Results } \\
\hline Sensor & Plataforma & Microcontrolador & Frequência & Rádio & SistemaOperativo & RSSI \\
\hline Sensor1 & TmoteSky_0 & MSP430 & $\mathrm{Ghz} 2.4$ & CC2420_0 & tinyos1 & -80 \\
\hline Sensor10 & MicaZ & MSP430 & Ghz2.4 & CC1000_0 & tinyos 1 & -60 \\
\hline Sensor 2 & Mica2 & ATmega_128 & Mhz1.8 & CC2420_0 & tinyos 2 & -90 \\
\hline Sensor3 & telosB_0 & MSP430 & $\mathrm{Ghz} 2.4$ & CC2420_0 & tinyos 2 & -85 \\
\hline Sensor 4 & TmoteSky_0 & MSP430 & Ghz2.4 & $\mathrm{CC} 2420$ & tinyos 1 & -90 \\
\hline Sensor5 & Mica2 & ATmega_128 & Mhz868 & CC1000_0 & mantis & -50 \\
\hline Sensor6 & Mica2 & ATmega_128 & Mhz868 & CC1000_0 & mantis & -50 \\
\hline Sensor 7 & MicaZ & ATmega_128 & Ghz2.4 & CC2420_0 & tinyos 2 & -60 \\
\hline Sensor8 & TmoteSky_0 & MSP430 & $\mathrm{Ghz} 2.4$ & CC2420_0 & tinyos 1 & -50 \\
\hline Sensor9 & telosB_0 & MSP430 & Ghz2.4 & CC2420_0 & tinyos 1 & -50 \\
\hline sensor11 & Mica2 & ATmega_128 & Mhz868 & CC1000_0 & mantis & -30 \\
\hline sensor12 & Mica_2dot & ATmega_128 & Mhz868 & CC1000_0 & tinyos 2 & -30 \\
\hline sensor13 & MicaZ & ATmega_128 & Mhz868 & CC1000_0 & mantis & -50 \\
\hline sensor14 & telosB_0 & MSP430 & $\mathrm{Ghz} 2.4$ & CC2420_0 & tinyos 2 & -26 \\
\hline sensor15 & Mica_2dot & ATmega_128 & Mhz868 & CC1000_0 & tinyos 2 & -30 \\
\hline sensor16 & TmoteSky_0 & MSP430 & $\mathrm{Ghz} 2.4$ & CC2420_0 & tinyos 2 & -35 \\
\hline
\end{tabular}

Figura 30 Dados dos Sensores 
Definiu-se seleção direta ao tipo de consulta feita no repositório de dados que retorna apenas quando o casamento é exato, ou seja, se o usuário precisa de temperatura do ambiente, mas se ele não conhece o termo exato e digita TEMPERATURA, o sistema não retorna nada. Quando o usuário busca por termos que são desconhecidos do repositório de dados, uma mensagem de erro é exibida, isto é considerada uma falha e é mostrada na Figura 33 como sendo zero.

Note na Tabela 1 que a busca direta com múltiplos recursos de hadware e software como, Plataforma:=TmoteSky. Freqüência:=2.4 Ghz, e RSSI $<=-50$ retorna três nós (nó1, nó4, nó8) de acordo com a Figura 30.

Tabela 1 Busca Direta: Plataforma:=TmoteSky. Freqüência:= 2.4 Ghz, e RSSI <= -50

\begin{tabular}{|c|c|c|c|}
\hline ID do Nó sensor & $\begin{array}{c}\text { Plataforma dos Nós } \\
\text { sensores }\end{array}$ & Freqüência & $\begin{array}{c}\text { Sinal de } \\
\text { RSSI }\end{array}$ \\
\hline Nó1 & $\begin{array}{c}\text { TmoteSky(TI MSP430, 2.4 } \\
\text { Ghz, CC2420) }\end{array}$ & $2,4 \mathrm{GHz}$ & -80 \\
\hline Nó4 & $\begin{array}{c}\text { TmoteSky(TI MSP430, 2.4 } \\
\text { Ghz, CC2420) }\end{array}$ & $2,4 \mathrm{GHz}$ & -90 \\
\hline Nó8 & $\begin{array}{c}\text { TmoteSky(TI MSP430, 2.4 } \\
\text { Ghz, CC2420) }\end{array}$ & $2,4 \mathrm{GHz}$ & -50 \\
\hline
\end{tabular}

No caso do usuário fazer uma busca semântica são exibidos os recursos semanticamente semelhantes ou equivalentes, ou seja, os mais parecidos com o que foi solicitado pelo usuário. Para isto, é feita uma verificação na ontologia com o intuito de descobrir os nós compatíveis com a requisição.

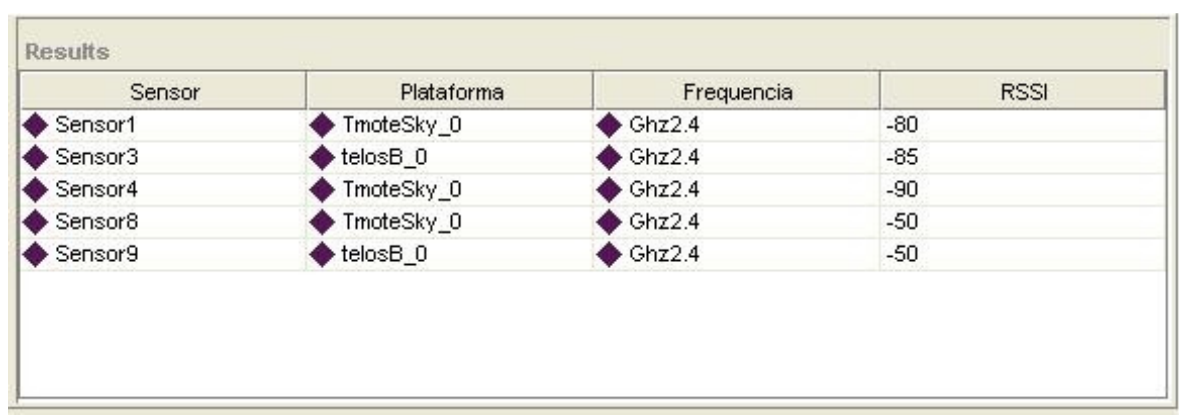

Figura 31 Busca Semântica: Plataforma:=TmoteSky. Freqüência:= 2.4 Ghz, e RSSI <= -50 
Já a busca semântica (Figura 31), retorna cinco nós (nó1, nó3, nó4, nó8, nó9), visto que TmoteSky é compatível com Telosb; para um análise de sinal dos nós sensores depende da freqüência e do padrão de comunicação, neste caso a freqüência é 2,4 Ghz e o padrão é ZigBee.

Considerando-se apenas um recurso e utilizando a busca direta, como S.O: TinyOS 1.x, o sistema retorna cinco nós mostrados na Tabela 2.

Tabela 2 Busca Direta: S.O.= tinyOS 1.x

\section{ID do Nó sensor Sistema Operacional}

\begin{tabular}{|c|c|}
\hline Nó1 & TinyOS 1.x \\
\hline Nó10 & TinyOS 1.x \\
\hline Nó4 & TinyOS 1.x \\
\hline Nó8 & TinyOS 1.x \\
\hline Nó9 & TinyOS 1.x \\
\hline
\end{tabular}

Repetindo-se esta mesma busca, mas de forma semântica, obtêm-se treze nós sensores mostrados na Figura 32; visto que existem aplicações que podem ser usadas tanto no TinyOS 1.x e TinyOS 2.0 como no oscilloscopeRF, Surge, entre outros.

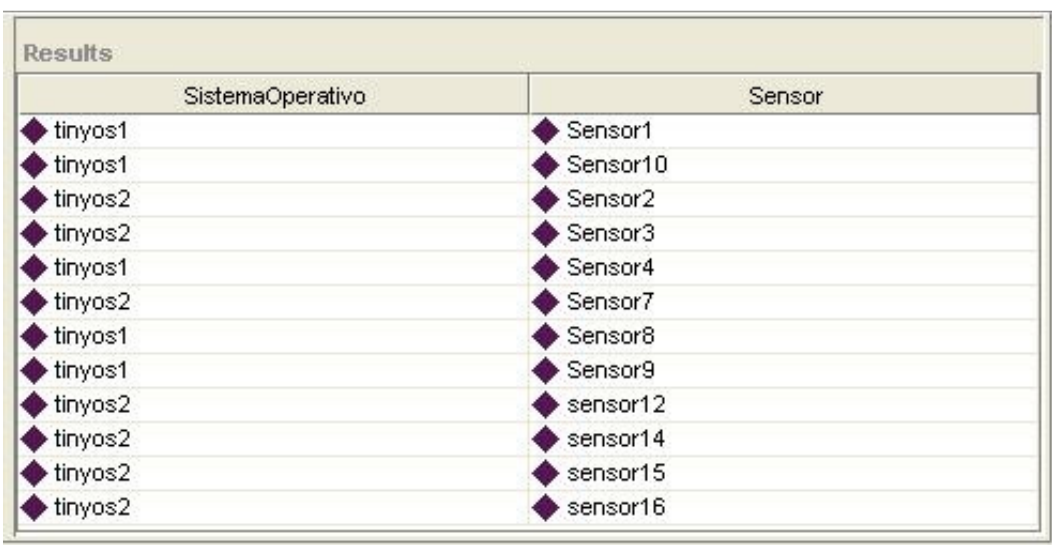

Figura 32 Busca Semântica: S.O.=tinyOS 1.x 


\subsection{Análises dos Resultados Obtidos}

Nesta subseção, comparamos duas abordagens distintas para a recuperação de informação no gerenciamento de dados da RSSF. A abordagem empregada usando um sistema de busca direta (base de dados convencionais $S Q L$, middleware para RSSF tinyDB) e a uma abordagem baseada em metadados semânticos.

Analisando os resultados obtidos no Teste1, podemos observar que uma das vantagens de gerenciamento de dados usando semântica no domínio de RSSF é a possibilidade de inferir novas informações, como ilustra na Figura 28. A comparação entre as diferentes abordagens é apresentada na Tabela 3 , onde se mostra a importância das técnicas semânticas na formação de associações entre os dados para adicionar riqueza na capacidade de consultas. As abordagens são qualificadas da seguinte forma:

$\checkmark$ - quando o critério de comparação é definido de forma adequada.

Em branco - quando não é considerado o critério de comparação.

Tabela 3 Diferentes Ferramentas para o Gerenciamento de dados

\begin{tabular}{|l|c|c|c|}
$\begin{array}{c}\text { Recuperação de } \\
\text { Informação }\end{array}$ & $\begin{array}{c}\text { Middleware RSSF } \\
\text { (tinyDB ) }\end{array}$ & $\begin{array}{c}\text { Ferramentas } \\
\text { (MSR sense ) }\end{array}$ & $\begin{array}{c}\text { Ontologias para } \\
\text { RSSF }\end{array}$ \\
\hline Consultas simples & $\checkmark$ & $\checkmark$ & $\checkmark$ \\
\hline $\begin{array}{l}\text { Consultas } \\
\text { compostas }\end{array}$ & & $\checkmark$ & $\checkmark$ \\
\hline $\begin{array}{l}\text { Inferência } \\
\text { semântica }\end{array}$ & & & $\checkmark$ \\
\hline
\end{tabular}

Analisando os resultados obtidos no Teste 2, na busca direta da consulta composta podemos observar que a taxa de acerto é bem baixa, procura identificar os nós sensores cuja plataforma seja descrita de modo idêntico à descrição da plataforma requisitada para observar o nível do sinal dos nós. Então, a busca direta retorna estritamente apenas aquilo que foi digitado pelo usuário (Figura 33).

Já na busca semântica permite de mais flexibilidade na locação de informação, aumentando o número de nós sobre os quais é possível analisar o sinal já que são equivalentes por ter as mesmas características. A taxa de acerto é bem 
acentuada e para os testes realizados não houve caso de falha, como se observa na Figura 34.

$\mathrm{Na}$ consulta simples onde se requerem os nós com sistema operacional TinyOS observa-se na Tabela 2 que a busca direta retorna só os nós sensores que contêm esse sistema operacional. Porém com a busca semântica consigo ter os sensores com um sistema operacional compatível, como é mostrado na Figura 32.

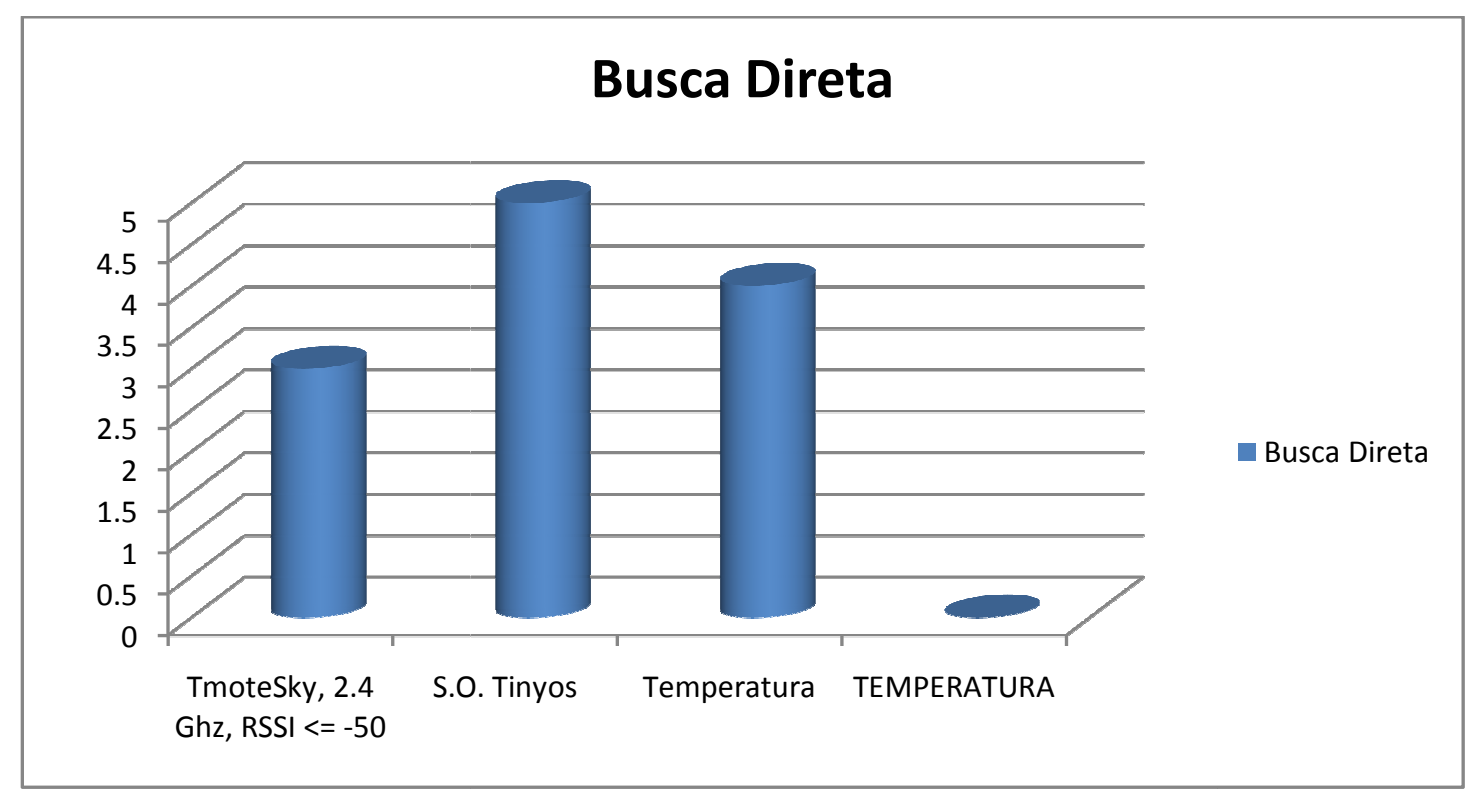

Figura 33 Resultados da Busca Direta.

É interessante observar que o número de nós de temperatura é o mesmo quando o nome é TEMPERATURA, veja Figura 35. Isto se deve ao fato da criação de relações de equivalência utilizando-se como base um dicionário de conceitos equivalentes (ou sinônimos). 


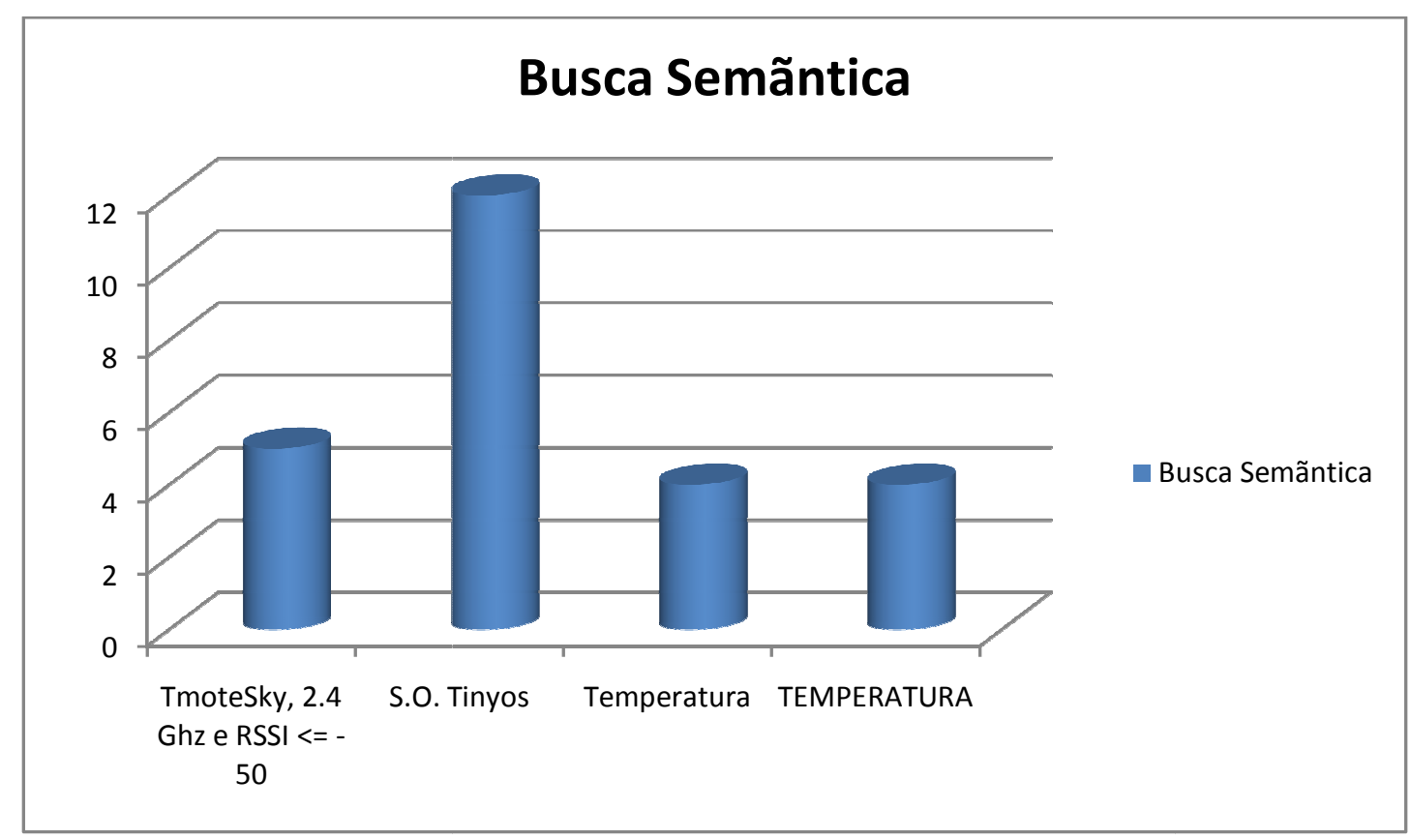

Figura 34 Resultados da Busca Semântica.

Assim, podemos comprovar a eficiência do tratamento semântico na Figura 34, uma vez que não só a quantidade de informação retornada é maior, mas também a qualidade da consulta; já que os nós considerados semanticamente equivalentes também estão aptos para a utilização pelo usuário, pois têm as mesmas características semânticas dos nós que foram requeridos. Observe na Figura 35 um comparativo entre o resultado da busca direta e semântica. De acordo com os testes realizados ficou comprovada a eficiência do tratamento semântico. 


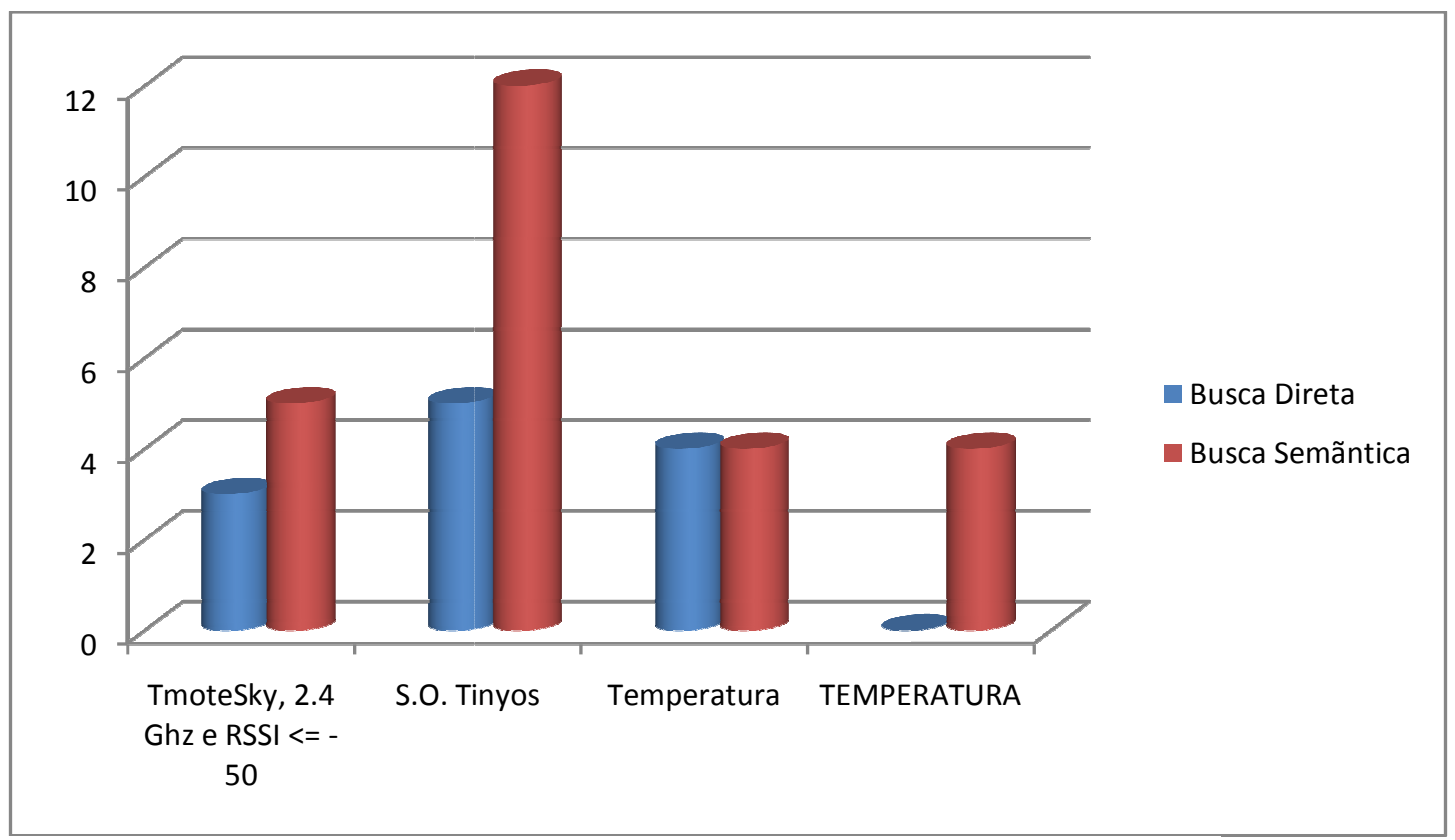

Figura 35 Resultados das Buscas Diretas vs. Semântica. 


\section{Capítulo 7}

\section{Considerações Finais}

\subsection{Contribuições}

As contribuições ao término deste trabalho utilizando nossa proposta no gerenciamento de dados nas redes de sensores sem fio são apresentadas a seguir:

- Ter um maior controle dos dados coletados nas RSSF.

- Converter os dados da rede em informações que sirvam ao usuário para tomada de decisões.

- Determinação específica das características gerais que devem ter as redes de sensores sem fio e suas aplicações (veículos, casas, pontes, etc.).

- O presente trabalho permite ser utilizado em distintos ambientes. O estudo do caso escolhido permite o gerenciamento dos dados em um ambiente automotivo onde se verifica o estado do veículo, recursos de rede, entre outros.

- Resolve o problema da fusão de dados no nível de aplicação de diferentes redes de sensores, de tal forma, que as aplicações possam realizar consultas significativas para resolver problemas do mundo real.

- Mostrar e determinar que através da semântica, as ontologias propostas sejam adequadas para o gerenciamento dos dados da RSSF. 


\subsection{Conclusões}

A partir dos resultados obtidos podemos extrair as seguintes conclusões:

- Este trabalho exemplifica como o uso da semântica pode realçar a gerência de dados em redes de sensores. Os relacionamentos subjacentes desta semântica entre os dados capturados pelos sensores criam uma estrutura versátil que pode ser utilizada em várias aplicações, como foi realizado no estudo de caso no Capítulo 6.

- Verificou-se que o uso de ontologias permite que os dados estejam bem definidos e bem situados no contexto e que dê como resultado um sistema de inferência confiável e eficiente, como demonstrado no Teste 1 (seção 6.2.1).

- A abordagem proposta baseada em ontologias e mecanismo de inferência mostrou-se útil nos cenários de recuperação de informações sobre os dados do ambiente monitorado e nos dados do nó sensor, visando um gerenciamento eficiente, como demonstrado no Teste 2 (seção 6.2.2).

- Verificou-se que a tendência dos middleware para gerência de dados das RSSF é usar abordagens semânticas e, assim, melhorar as técnicas que existem atualmente. Acreditamos que uma nova geração de DBMS requer sustentações semânticas adicionais para uma gerência de dados flexível e eficiente, como demonstrado nos testes 1 e 2 .

- Outro aprimoramento semântico é que as palavras sinônimas de linguagem fossem tratadas pelo template ontológico. Por exemplo, TEMPERATURA e temperatura representariam o mesmo conceito ontológico. Assim, espera-se que a descoberta semântica de dados e recursos apresentem um desempenho progressivo.

- Verificou-se, que o sistema proposto é uma maneira útil de monitorar veículos. Preveria problemas com antecedência, reduziria custo de manutenção, pois os erros inerentes às revisões periódicas serão reduzidos, e auxiliaria nas situações de emergência. 


\subsection{Limitações}

Neste trabalho temos algumas limitações relacionadas à facilidade do usuário. Realizamos todos os testes utilizando o Pluging da linguagem SPARSQL no Protégé, mas pode-se realizar uma interface mais simples e funcional que permita realizar as consultas de uma maneira mais ótima. Um browser pode ser criado para a navegação na ontologia dos dados da RSSF, facilitando o trabalho do pesquisador, que será o usuário final do sistema.

\subsection{Trabalhos Futuros}

- Integração dos agentes e da ontologia proposta na plataforma JADE para otimizar o gerenciamento dos dados de apoio às decisões estratégicas tomadas no ambiente pervasivo.

- Os aspectos associados ao relacionamento semântico entre ontologias com intersecção de domínio, mapping, e entre ontologias de domínios disjuntos, econnections, precisam ser mais explorados para promover a escalabilidade do modelo em padrões mais elaborados.

- Outra funcionalidade a ser acrescida, é a possibilidade da realização de matching baseado em um grau de similaridade entre conceitos.

- Criar um middleware para RSSF que permita o gerenciamento dos dados com uma abordagem semântica. 


\section{Referências}

AKYILDIZ, I.; WELLJAN, S.; SUBRAMANIAM, Y.; CAYIRCI, E. A survey on sensor networks. Communications Magazine IEEE, v. 40, 8, p. 102-114, Aug. 2002.

ARAÚJO, F. C.; ANDERSON F.; KATYUSCO DE F. S. Avaliação de Estratégias e Construção de Software para Medição do Nível de Energia em Sensores de Redes de Sensores sem Fio (RSSF), II Congresso de Pesquisa e Inovação da Rede Norte Nordeste de Educação Tecnológica, Pernambuco, 2007.

BALAZINSKA, M. et al. Data Management in the Worldwide Sensor Web. IEEE Pervasive Computing. v. 6, 2, p. 30-40 Apr-Jun, 2007.

BALDONI, R.; CORDESE, G.; DAVIDE, F., MELPIGNANO, Data management in wireless sensor network. In: Global Data Mangement, IOS Press, 2006, p. 46-56. Disponível em:<http://books.google.com.br/books?id=ot5w8P5ueqgC >. Acesso em: 03 Jun. 2008

BASAGNI, S.; GIORDANO, M.; CONTI, S.; STOJMENIVIC, I. Mobile Ad Hoc Networking. In: IEEE Press and John Wiley and Sons, Inc., New York, 2004.

BECHHOFER, S. et al. OWL Web Ontology Language Reference, Feb. 2004. Disponível em :< http://www.w3.org/TR/2003/CR-owl-features-20030818/>. Acesso em: 15 Mai. 2008.

BECHHOFER, S.; LORD, P.; VOLZ, R.; Cooking the semantic web with the owl api. In: Proceedings of the 2nd. Int. Semantic Web Conference (ISWC 2003), Sanibel Island, Anais....Florida, USA, Oct.2003.

BECHHOFER, S.; MOLLER, R.; CROWTHER, P. The dig description logic interface. In: Proceedings of the Int. Description Logics Workshop, DL'2003. Anais..., 2003.

BERNERS-LEE, T. World wide web consortium. Disponível em:< http://www.w3.org/ >, Acesso em: 08 de Mai 2008.

BODROZIC, L.; STIPANICEV, D.; KRSTINIC, D. Data fusion in observer networks, Edição: Lj. Bodrožić. Mobile Adhoc and Sensor Systems, In:The Fourth IEEE International Conference on Mobile Ad-hoc and Sensor Systems MASS 2007. Anais... IEEE International Conference , 2007,Oct. p. 1-6.

CARROLL, J. J. et al. Jena: Implementing the semantic web recommendations. In: Proceedings of the 13th. Int. World Wide Web Conference (WWW 2004), New York Anais: New York ACM Press , 2004 , p 74-83.

CHEONG, E.; KANSAL, A.; LIU J.; OISON, T.; PRIYANTHA, B.; SETH, S. Networked Embedded Sensing Toolkit (MSR Sense). 16 de Dec de 2005. Disponível em: <http://research.microsoft.com/nec/msrsense/ >, Acesso em : 15 de Mai de 2008.

CHIMAERA. The chimaera ontology environment. Knowledge Systems Al. Knowledge Systems Al Laboratory Stanford University (2005) Disponível em: < 
http://www.ksl.stanford.edu/software/chimaera/>. Acesso em: Acesso em: 15 de Maio 2008.

CHISTOPHER, H.; CAMPBELL, R. H. An application of a context-aware file system. Personal Ubiquitous Comput, Springer, London, v. 7, 6, p. 339-352, 2003.

CONNOLLY, D.; HARMELEN, F.; HORROCKS, I.; McGUINNESS, D.; PALTESCHNEIDER, P.; STEIN, L. Daml+oil (março 2001) reference description. Disponível em :< http://www.w3.org/TR/daml+oil-reference >. Acesso em: 15 de Mai de 2008.

CONSORTIUM. W.W.W.W. Web Ontology Language (OWL), 2007, Disponível em: <http://www.w3.org/2004/OWL>. Acesso em: 15 de Mai de 2008.

CORCHO, Ó.; FERNANDEZ, L.; GOMEZ-PEREZ, A. Methodologies, tools and languages for building ontologies: where is their meeting point?. Data Knowl Eng. (Elsevier Science Publishers B. V), v.36, 1, p. 41-64, Amsterdam, 2003.

DEAN, M.; SCHREIBER, G. Owl web ontology language reference. W3C Recommendation. 10 de February de 2004. Disponível em:<http://www.w3.org/tr/owlref/>. Acesso em: 15 de Mai de 2008.

DEEPAK, G.; GREENSTEIN, B.; ESTRIN, D.; HEIDEMANN, J.; RAMESH, G. Multiresolution storage and search in sensor networks. Trans. Storage, v.1, 3, p. 277-315, New York, 2005.

DELICATO, C. D. Middleware baseado em serviçõs para Rede de sensores sem fio. 2005, 209 p. Teses (Doctorado), Engenharia Elétrica, Universidade Federal de Rio de Janiero, Rio de Janeiro, 2005.

DELICATO, F. C.; PIRMEZ, L.; PIRES, P. F.; FERREIRA, J. Exploiting Web Technologies to build Automic Wireless Sensor Networks. In: 8th IFIP/IEEE International Conference on Mobile and Wireless Communication (MWCN 2006),Santiago Anais...Santiago: IEEE International Conference ,2006 ,v.1, p. 99114.

DEVEDZI, VLADAN. Understanding ontological engineering. Commun. ACM, v. 45, 4, p. 136-144, New York, NY, USA, 2002.

DJURIC, D.; GASEVIC, D.; DEVEDZIC, V. Ontology modeling and MDA. Journal of Object Technology, v. 4, p. 109-128, Jan-Feb 2005.

DODDS, L. Introducing SPARQL: Querying the Semantic Web. 2006. Disponível em :<http://www.xml.com/pub/a/2005/11/16/introducing-sparqlquerying-semantic-webtutorial.html>. Acesso em: 15 de Mai de 2008.

EID, M.; LISCANO, R.; SADDIK, A. A Universal Ontology for Sensor Networks Data. In: Computational Intelligence for Measurement Systems and Applications( CIMSA 2007), Anais...Washington : IEEE International Conference on Jun 2007, p. 59-62. 
FERREIRA, L.; VAN, M.; MUNTHE-KAAS, E.; POKRAEV, S.; HUTSCHEMAEKERS, M.; PLAS, J. Techniques for describing and manipulating context information. Freeband A-MUSE project partners, Set 2005.

FIKES, R.; HAYES, P.; HORROCKS, I. OWL-QL: A Language for Deductive Query Answering on the Semantic Web, Knowledge Systems Laboratory, Stanford University, Stanford, 2003.

FLORIDO, I. R. Rede de sensores sem fio em ambientes veiculares baseada no padrão Zigbee. 2008, 121p. Dissetação (Mestrado),Escola Politecnica, Universidade de São Paulo, 2008.

GEHRKE, J.; MADDEN, S. Query Processing in Sensor Networks. IEEE Pervasive Computing, v.3,1,p. 46-55, 2004.

GENESERETH, M.R. Knowledge interchange format (kif). 2006. Disponível em :< http://logic.stanford.edu/kif/ >. Acesso em 15 de Mai de 2008.

GÓMEZ, A.; CORCHO, O. Ontology languages for the Semantic Web. Intelligent Systems, IEEE, v. 17, 1, p. 54-60, 2002.

GREENSTEIN, B.; ESTRIN, D.; GOVINDAN, R.; RATNASAMY, S.; SHENKER, S. DIFS: a distributed index for features in sensor networks, Sensor Network Protocols and Applications. In: First IEEE International Workshop on Sensor Network Protocols and Applications,2003, Anais... Washington: IEEE International Workshop, 2003, May, p.163-173.

GRUBER, THOMAS R. A translation approach to portable ontology specifications. Knowl. Acquis, v. 5, 2, p. 199-220, London, 1993.

GUARINO, NIKOLA. Formal Ontology and Information Systems. In: FOIS'98: Proceedings of the International Conference on Formal Ontology in Information Systems, Trento, Anais...Italy: 6-8 de Jun de 1998, p. 3-15.

HEIDEMANN, J. Building Efficient Wireless Sensor Networks with Low-Level Naming. In: SOSP '01: Proceedings of the eighteenth ACM symposium on Operating systems principles, Canada, Anais... New York, NY, USA :ACM, 2001, p. 146-159.

HENRICKSEN; INDULSKA, J. Developing context-aware pervasive computing applications:Models and approach, Journal of Pervasive and Mobile Computing, p. 37-64, feb 2006.

HENRICKSEN, K.; ROBINSON, R. A Survey of Middleware for Sensor Networks: State of the Art and Future Directions. In MidSens '06: Proceedings of the international workshop on Middleware for sensor networks Melbourne, Australia, Anais... New York, NY, USA :ACM Press 2006, p.60-65. 
HILL, J.; SZEWCZYK, R.; WOO, A.; HOLLAR, S.; CULLER, D.; PISTER, C. System architecture directions for networked sensors, SIGPLAN Not, v.34, 5, p.93-104.

HORRROCKS, I. 2003. Disponível em:<http://www.cs.man.ac.uk/ horrocks/FaCT/>. Acesso em 15 de Mai de 2008.

HP, Labs Semantic Web Research. Jena: A semantic web framework for java. 2006. Disponível em: <http://jena.sourceforge.net/>. Acesso em 15 de Mai de 2008.

OCML. Operational conceptual modelling Language. Institute Knowledge Media,2006. Disponível em< http://kmi.open.ac.uk/projects/ocml/>. Acesso em: $15 \mathrm{de}$ Mai de 2008.

INTANAGONWIWAT, C.; GOVINDAN, R.; ESTRIN, D. Directed diffusion: A scalable and robust. In MobiCom '00: Proceedings of the 6th annual international conference on Mobile computing and networking, Boston, Massachusetts, United States Anais..., New York: ACM, 2000. p. 56-67.

ISAAC, A.; TRONCY, R. Doe-the differential ontology editor, 2006. Disponível em: <http://homepages.cwi.nl/ troncy/DOE >. Acesso em 15 de Mai de 2008.

JURDAK, R.; VIDEIRA, C.; BALDIY. P. A Framework for Modeling Sensor Networks. Proceedings of the Building Software for Pervasive Computing Workshop, Anais... OOPSLA 2004.

KEPHART, J.; CHESS, D. M. The vision of autonomic computing. IEEE Computer Society Press, Los Alamitos, USA, v. 36, 1, p. 41-50, 2003.

KIFER, M., LAUSEN, G.; WU, J. Logical foundations of object-oriented and framebased languages. Technical Report, University of Mannheim, 1990.

KNUBLAUCH, H.; FERGERSON, R. W.; NOY, N. F.; MUSEN, M. A. Protégé owl plugin: An open development environment for semantic web applications, In ISWC: Third International Semantic Web Conference - Hiroshima, Anais... Hiroshima: 2004.

LASSILA, O.; SWICK, R. Resource description framework (rdf) - model and syntax specification. W3C Recommendation. 1999. Disponível em: http://www.w3.org/TR/REC-rdf-syntax/. Acesso em 15 de Mai de 2008.

LEWIS, M.; CAMERON, D.; XIE, S.; ARPINAR, B. ES3N: A Semantic Approach to Data Management in Sensor Networks. In SSN06: 5th International Semantic Web Conference, Georgia, Anais... Athens, Georgia, USA 2006.

LI, Y.; WANG, F.; HE, F.; LI, Z. OSGi-based service gateway architecture for intelligent automobiles Intelligent Vehicles Symposium, 2005. Proceedings. IEEE, Anais... Jun 2005, p. 861-865.

LOUREIRO, A.; RUIZ, B.; NOGUEIRA, J.; MINI, R. Rede de Sensores Sem Fio. Simpósio Brasileiro de Computação. Jornada de Atualização de Informática Anais... SBC, 2002, p. 193-234. 
LOUREIRO, A.; NOGUEIRA, J.; RUIZ, L. B.; NAKAMURA, E. F.; FIGUEIREDO, C. M. Redes de Sensores Sem Fio. XXI Simpósio Brasileiro de Redes de Computadores, Anais..., SBC, 2003, p. 48.

MADDEN, S. R.; FRANKLIN, M. J.; HELLERSTEIN, J. M.; HONG WEI. TinyDB: an acquisitional query processing system for sensor networks.New York. Anais..., New York : ACM Trans. Database Syst, 2005, v. 30,1 , p. 122-173.

MCCARTHY, P. Search RDF data with SPARQL. 2005. Disponível em :<http://www.ibm.com/developerworks/xml/library/j-sparql/>. Acesso 15 de Mai 2008.

MIZOGUCHI, R. Tutorial on ontological engineering - part 2: Ontology development, tools and languages. New Generation Computing, v. 22, n. 1, p.61-96, 2004. Disponível em: <http://www.ei.sanken.osaka-u.ac.jp/pub/miz/Part2V3.pdf>. Acesso 15 de Mai 2008.

MOTEIV. Tmotesky. ultra low power ieee 802.15.4 compliant wireless sensor module. Disponível em: www.eecs.harvard.edu/ konrad/projects/shimmer/references/ tmote-sky-datasheet.pdf>. Acesso em 15 de Mai de 2008.

NECIB, C. B.; FREYTAG, J C. Ontology Based Query Processing in Database Management Systems. CoopIS/DOA/ODBASE, 2003,p. 839-857.

NI, LIONEL M., et al. Semantic Sensor Net: An Extensible Framework. International Journal of Ad Hoc and Ubiquitous Computing (IJAHUC), 2006.

NOY, N.; FERGERSON, R.; MUSEN, M. The knowledge model of protege. Lecture notes in computer science, 2000.

NOY, N. F.; MCGUINNESS, D. L. Ontology Development 101: A Guide to Creating Your First Ontology. Stanford University, Stanford, 2001.

O'DONOGHUE, J.; HERBERT, J. An Intelligent Data Management Reasoning Model within a Wireless Patient Sensor Network. Proceedings of Artificial Intelligence Techniques for Ambient Intelligence (AITAml'06) in conjunction with ECAI 2006, Anais... Riva de Garda, Italy, Aug 2006a.

O'DONOGHUE, J.; HERBERT, J. A QoS Data Management System within a Pervasive Medical Environment. Proceedings of the Doctor Colloquium in conjunction with Pervasive, , Anais... Dublin, May 2006b.

O'DONOGHUE, J.; HERBERT, J. Profile based sensor data acquisition in a ubiquitous medical environment. In PerCom: Pervasive Computing and Communications Workshops, Fourth Annual IEEE International Conference on. Anais... 2006c, 17 Mai, p. 5.

O'DONOGHUE, J.; HERBERT, J. Data Management System: A Context Aware Architecture For Pervasive Patient. Proceedings of the 3rd International Conference 
on Smart Homes and Health Telematic (ICOST 2006), Anais... Northern Ireland, 2006c, p. 159-166.

OMG. UML Resource Page. Object Management Group. 2008. Disponível $<$ http://www.uml.org/>. Acesso em 15 de Mai de 2008.

ONTOLINGUA. Ontolingua Software Description. Stanford University (2005) Disponível em: < http://www.ksl.stanford.edu/software/ontolingua/>. Acesso em: Acesso em: 15 de Mai 2008.

ONTOPRISE. Ontostudio. 2008.2 Disponível em:<http://ontoedit.com/index.php?id=179. Acesso em 15 de Mai de 2008.

PELLET. Pellet owl reasoner, UNIVERSITY OF MARYLANDS 'S LAB. 2006. Disponível em < http://www.mindswap.org/2003/pellet/>. Acesso 15 de Mai 2008.

PEREIRA, MARLUCE R., CLAUDIO L. AMORIM, e MARIA STELLING. Tutorial sobre Redes de Sensores. In Simpósio Brasileiro de Redes de Computadores (SBRC), 2004.

PINTO, A. J. G. Mecanismo de Agregação de Dados baseado em Técnicas Paramétricas aplicado em Redes de Sensores. 2004. p. 115, Dissertação (Mestrado), Engenharia Elétrica, UFRJ, Rio de Janeiro.

PIRMEZ, L.; DELICATO, F. C.; MOSTARDINHA, A. L.; REZENDE, N. Uma abordagem baseada em Lógica Nebulosa para a Seleção de Protocolos de Disseminação de Dados em RSSFs. Anais...,SBRC, 2006.

PROJECT LOOM, Loom project home page, University of Southern California's Information Sciences Institute (2006). Disponível em: http://www.isi.edu/isd/LOOM/. Acesso em: 15 de Mai 2008.

PROTÉGÉ. The protégé ontology editor and knowledge acquisition system. Stanford Medical Informatics at the Stanford University School of Medicine.2006. Disponível em:

$<$ http://protege.stanford.edu/>. Acesso 15 de Mai 2008.

RAGHUNATHAN, V.; SPANOS, P.; SRIVASTAVA, M. Adaptive power-fidelity in energy aware wireless embedded systems. Proceeding Real-Time Systems Symposium, 2001. Anais..., RTSS, 2001,Dec, p. 106-115.

RATNASAMY, S., et al. a geographic hash-table for data-centric storage. In Workshop on Wireless Sensor Networks and Their Applications, Atlanta, Anais... Georgia: ACM International, 2002, Sep.

RECTOR, A., et al. OWL Pizzas: Practical Experience of Teaching OWL-DL: Common Errors \& Common Patterns. Engineering knowledge in the age of the semanticweb Springer, p. 63-81, Berlin, 2004. 
RECTOR, A.; DRUMOND, N.; HORRIDGE, M.; ROGERS, J.; KNUBLAUCH, H.; STEVENS, R.; WANG, H.; OWL Pizzas: Practical Experience of Teaching OWL-DL: Common Errors \& Common Patterns. Proceedings of the European Conference on Knowledge Acquistion, Northampton, England, 2004, Anais... Lecture Notes on Computer Science. Springer, 2004, p. 63-81.

RUIZ, L., et al. Arquiteturas para redes de sensores sem fio. Simpósio Brasileiro de Redes de Computadores .Anais... SBRC, 2004.

RUSSOMANNO, D. J.; KOTHARI, C. R.; THOMAS, O. A. Building a Sensor Ontology: A Practical Approach Leveraging ISO and OGC Models. In: International Conference on Artificial Intelligence, Las Vegas, Anais... Las Vegas, 2005, p. 637643.

SHIH, E.; CHO, S. H.; ICKES, N.; MIN, R.; SINHA, A.; WANG, A.; CHANDRAKASAN, A.; Physical Layer Driven Protocol and Algorithm Design for Energy-Efficient Wireless Sensor Networks. MobiCom '01: Proceedings of the 7th annual international conference on Mobile computing and networking (ACM), New York , Anais... New York: ACM International,2001, p. 272-287.

SILVA, FABRÍCIO AGUIAR. Avaliação de abordagens de Gerenciamento para Redes de Sensores sem Fio.2006. p. 101, Dissertação (Mestrado), Instituto de Ciências Exatas, Universidade Federal de Minas Gerais, Minas Gerais, 2006.

SIRIN, E.; PARSIA, B. Pellet: An owl dl reasoner. In: Volker Haaslev, R.M. (Ed.), Whistler, Proceedings of the International Workshop on Description Logics ,2004, Anais... Canada, Jun 6-8, v.104, 2004.

SOWA, JOHN F. Knowledge Representation: Logical, Philosophical, and Computational Foundations. Pacific Grove, Brooks Cole Publishing, p.534, California, 1999.

SU, X.; ILEBREKKE, L. A Comparative study of ontology languages and Tools. Proceedings of the 14th International Conference on Advanced Information Systems Engineering, Springer, Anais... London: CAiSE , 2002, p. 761-765.

SUN MICROSYSTEMS. Java architecture for xml binding. 2006. Disponível em:< http://java.sun.com/xml/downloads/jaxb.html>. Acesso em: Maio de 2008.

RACER SYSTEMS. Racerpro reasoner. 2006. Disponível em:< http://www.racersystems.com/ > Acesso em Maio de 2008.

TILAK, S.; ABU-GHAZALEH, N. B.; HEINZELMAN, W. Infrastructure tradeoffs for sensor networks. WSNA '02: Proceedings of the 1st ACM international workshop on Wireless sensor networks and applications, New York, Anais... New York: ACM Press,2002,

TINYOS. Fifth International TinyOS Technology Exchange (TTX5). University of Berkeley. 23 de set. 2003. Disponível em:< http://www.tinyos.net/tinyos1.x/doc/tutorial/lesson6.html>. Acesso em: 03 de June 2008. 
TILAK, S.; ABU-GHAZALEH, N. B.; HEINZELMAN, W. A taxonomy of wireless microsensor network models. SIGMOBILE Mob. Comput. Commun. Rev. (ACM Press), v. 6,2 , p. 28-36, 2002.

TSENG, Y. C.; PENG, W. C.; LEUNG, V.; CHEN, W.T.; PINOTTI, C. Information processing and data management in wireless sensor networks. Elsevier. v. 87, Dec. 2007.

ULIERU, M.; MADANI, S.A. An Application of Industrial Agents to Concrete Bridge Monitoring. Proceedings of ICINCO 2006 - 3rd International Conference of Informatics in Control, Automation and Robotics, Setubal, Anais.... International Conference ICINCO 2006, Aug 1-5, p. 43-50.

WALSH, N. A Technical Introduction to XML. October 1998. Disponível em: <http://www.xml.com/pub/a/98/10/guide0.html>. Acesso em: 03 de Jun 2008.

WOO, A.; SETH, S.; OLSON, T.; LIU, J.; ZHAO, F. A Spreadsheet Approach to Programming and Managing Sensor Networks. IPSN '06: Proceedings of the fifth international conference on Information processing in sensor networks Anais... ACM Press, 2006, p. 424-431.

WUN, A.; PETROVI, M.; JACOBSEN, H. A. A system for semantic data fusion in sensor networks. In DEBS '07: Proceedings of the 2007 inaugural international conference on Distributed event-based systems. Toronto, Anais... York, NY, USA: ACM. 2007, p. 75-79.

XU, NING, RANGAWALA, S., CHINTALAPUDI, K. N., D. GANESAN, A., GOVINDAN, R. BROAD, e D. ESTRIN. A wireless sensor network for structural monitoring. In SenSys '04: Proceedings of the 2nd international conference on Embedded networked sensor systems, Baltimore, MD, USA, 2004, Anais... New York, NY, USA: ACM, p. 13-24. 2004.

XUE, GUANGTAO, QUNHUA PAN, e MINGLU LI. A New Semantic-based Query Processing Architecture. ICPPW '07: Proceedings of the 2007 International Conference on Parallel Processing Workshops, Anais... IEEE Computer Society, 2007, p. 63.

ZHI-FENG, D.; YUAN-XIANG, L.; GUO-LIANG, H.; YA-LA, T.; XIAN-JUN, S. Uncertain Data Management for Wireless Sensor Networks Using Rough Set Theory. Wireless Communications, Networking and Mobile Computing, Anais...WICON, 2006,Set. p.1-5.

ZOU, Y.; FININ, T.; CHEN , H. F-owl: an inference engine for the Semantic web. Lecture Notes in Computer Science, p. 238-248. 2005.

ZUÑIGA, J. C.; ROCA, I.; KOFUJI, S. T. Modelagem de Dados e Sensores para aplicações em Intra-Vehicle Wireless Sensor Networks In: I Workshop on Pervasive and Ubiquitous Computing, 2007, Anais... Gramado, 2007. 


\section{Anexos}

\section{A Anexo A - Arquitetura e Implementação}

Este Anexo descreve a arquitetura proposta para a efetivação do sistema e alguns detalhes de maior relevância sobre sua implementação.

A Figura 36 mostra a arquitetura da abordagem semântica para RSSF proposta. A arquitetura tem cinco camadas, que serão descritas a seguir.

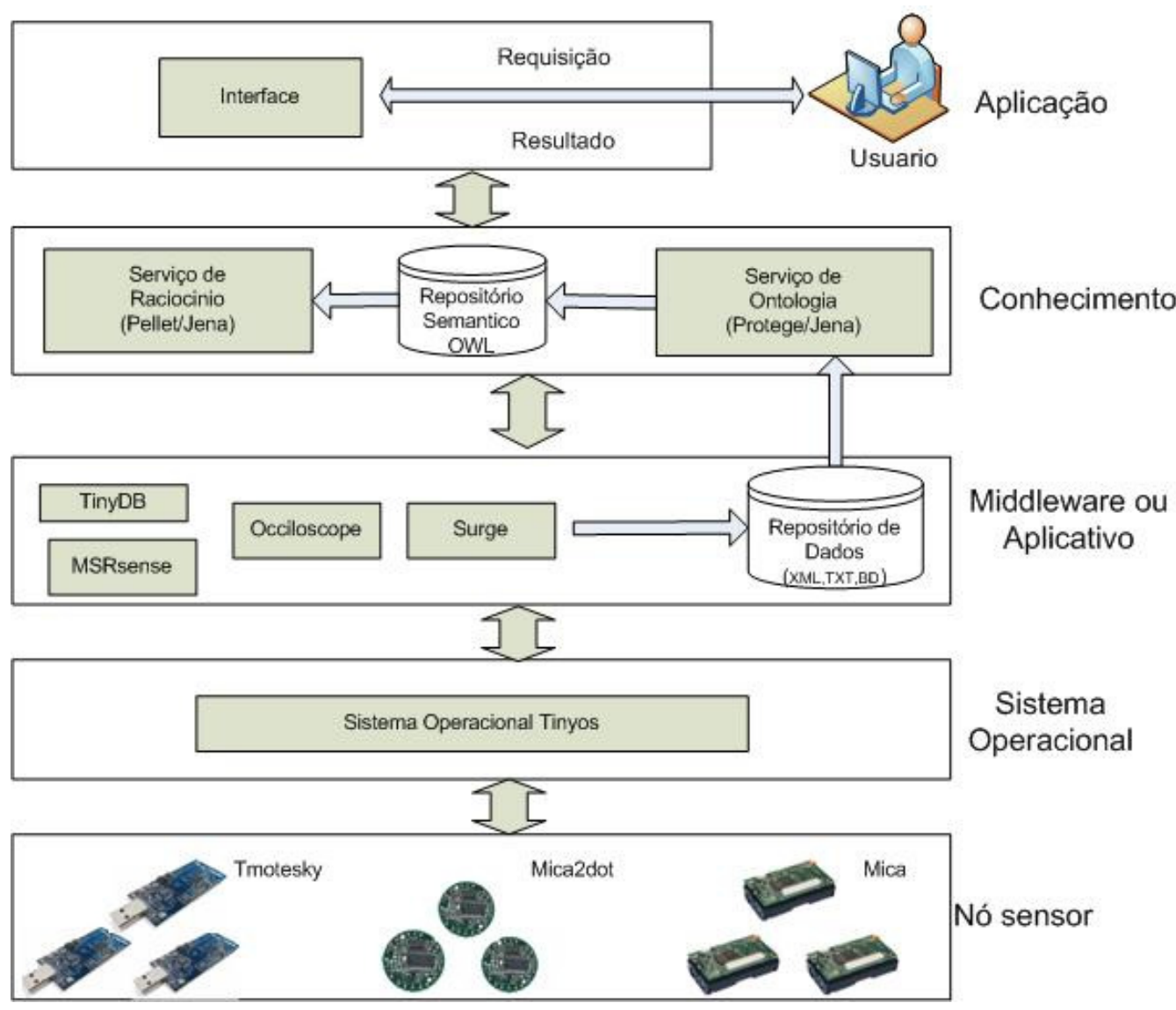

Figura 36 Arquitetura de Cinco Camadas da abordagem Semântica para RSSF

A camada física está diretamente ligada às distintas plataformas de redes de sensores e seus recursos de hardware, software, visto na Tabela 4 (Anexo B).

A camada de sistema operacional refere-se ao sistema operacional que vai ser instalado sobre a plataforma dos nós sensores; o mais comum é o TinyOS descrito no Anexo B. 
A camada do middleware ou aplicativo permite o acesso aos dados, nesta camada os dados captados pelos nós podem ser armazenados em arquivos TXT, XML ou base de dados, como se mostra na Figura 36.

A camada de conhecimento é o foco principal deste trabalho e provê o serviço de gerenciamento de dados das RSSF de uma grande quantidade de dados capturados pelos sensores armazenados no repositório de dados. Para capturar as informações dos nós e transferi-las para o repositório semântico, na linguagem de ontologia Web OWL (DEAN; SCHREIBER, 2004), foi utilizado o Jena, que é um framework para aplicações de Web Semântica. O Jena é um software de código aberto e provê um ambiente programacional para OWL e uma API OWL. O projeto Jena surgiu como um programa de Web Semântica dos laboratórios da HP (HP 2006).

A arquitetura do Jena provê um mecanismo para anexar raciocinadores externos aos modelos Jena, através da reimplementação da interface gráfica. A versão 2.1 do Jena provê uma ilustração desta técnica provendo um gateway transparente entre os modelos de ontologia do Jena e raciocinadores externos implementando a interface de descrição lógica do raciocínio ou Description Logics Interface (DIG).

A camada de conhecimento é formada pela ontologia e os componentes de raciocínio, como já apresentado na Figura 36. Os componentes implementados na camada de conhecimento são: o componente ontológico onde utilizamos o Protégé (seção 3.2.3) e o componente raciocinador que utiliza o motor de inferência Pellet (seção 3.3), e que pode ser utilizado juntamente com o Jena ou com bibliotecas da API OWL.

E, finalmente, a camada de aplicação é a que fica no topo da arquitetura, a qual provê que o requisitante de usuário submeta uma consulta e obtenha o resultado semântico de sua requisição através do serviço de ontologia que utiliza o motor de inferência. 


\section{A.1 Aspectos da Implementação}

Será descrito o workflow de execução para a elaboração de um estudo de caso usando a abordagem semântica para o gerenciamento dos dados, primeiramente foram construídas as ontologias, onde foi utilizada a metodologia de desenvolvimento 101 (NOY; MCGUINNESS 2001) que consta de sete passos desenvolvidos iterativamente, apresentado no Capítulo 5. Neste ponto, a ontologia com os conceitos e propriedades relacionadas forma o repositório semântico na linguagem OWL.

Após as ontologias serem definidas, o serviço de gerenciamento de dados coleta os dados utilizando o MSRsense e salva dinamicamente os dados reais do ambiente relacionados a um determinado instante, a instalação é apresentada no anexo C. Estes dados foram salvos no repositório de dados utilizando-se a linguagem XML com a informação provida pelo serviço do MSRsense mostrada na Figura 37.

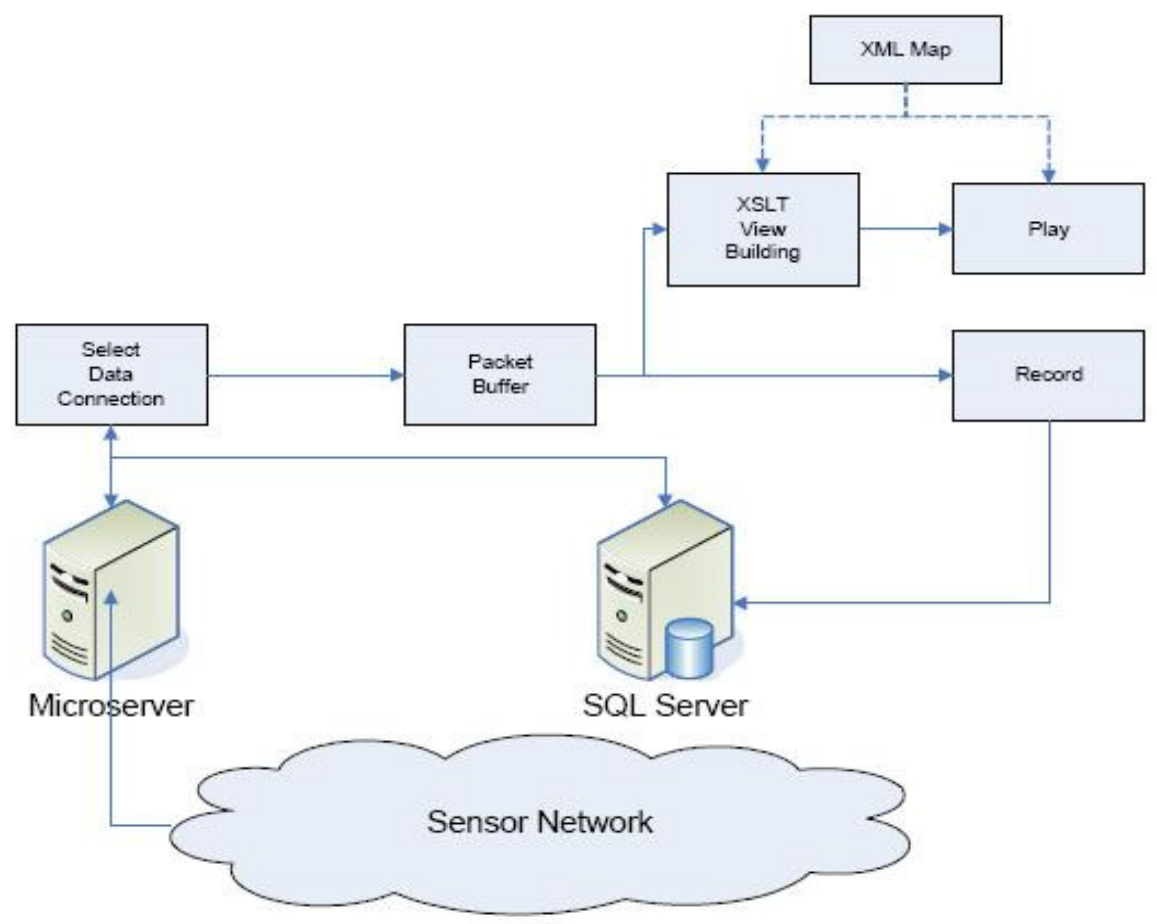

Figura 37 esquema do tratamento de dados usando MSRsense (WOO et al., 2006) 
De modo a instanciar a ontologia, nós primeiramente fazemos um bind dos dados dos nós definidos em XML para classes Java utilizando a Java Architecture for XML Binding $(\mathrm{JAXB})^{10}$, que provê um modo conveniente de se fazer o bind do schema XML para sua representação em código Java e está disponível no Java Web Services Developer Pack WSDP 1.6 (SUN MICROSYSTEMS, 2006) .

Em seguida, para instanciar os dados do repositório semântico com as ontologias propostas, precisa-se carregar a ontologia criada no Protégé à biblioteca Jena, o procedimento se mostra na seguinte seção.

\section{A.1.1 Implementação em Java}

Para a manipulação dos dados utilizando dispositivos reais e sua integração com as ontologias propostas, devem ser construídas interfaces implementadas em Java. Inicialmente devem ser importadas as bibliotecas Java necessárias, posteriormente criar uma pasta (File Data) que contém as ontologias propostas criadas como o Protégé, e finalmente o código Java de implementação.

\section{- Definir as classes e hierarquias de classes}

Para implementar em Java o Estudo de Caso devem ser importadas as bibliotecas de Jena e Pellet. A versão utilizada do Jena 2.5.2 a versão de Pellet é 1.5.1. A Figura 38 mostra o projeto das bibliotecas necessárias.

\footnotetext{
${ }^{10}$ API Java Architecture for XML Binding (JAXB) provê ferramentas e mecanismos que automatizam o mapeamento de documentos XML em objetos Java.
} 


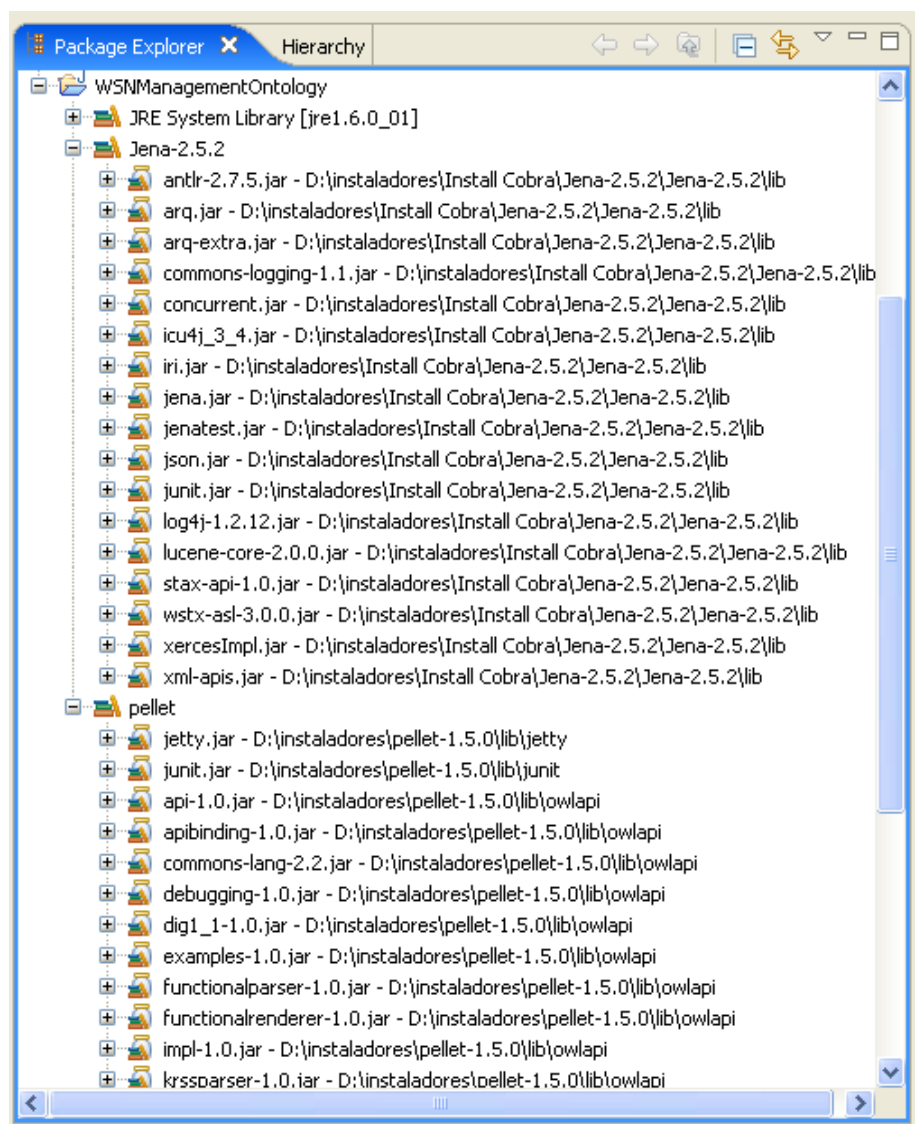

Figura 38 Bibliotecas necessárias para a implementação de Estudo de Caso

- Gerar Java Schema class

Uma vez importadas as bibliotecas necessárias, em seguida tem que ser gerado a Java Schema class utilizando o Protégé, como mostra-se na Figura 39, para ser importado desde o Eclipse e obter o vocabulário das ontologias (Vocab), mostrado na Figura 40.

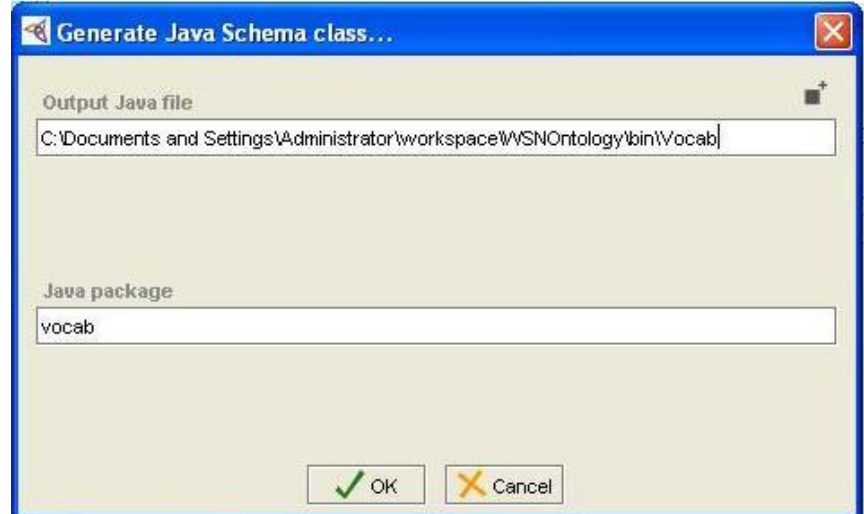

Figura 39 Janela para gerar Java Schema Class - utilizando o Protégé 
- Criar uma pasta com as ontologias

Posteriormente tem que ser criado uma pasta (Data) com as ontologias propostas. Na Figura 40, é apresentado a pasta que contém as ontologias do projeto WSNOntology

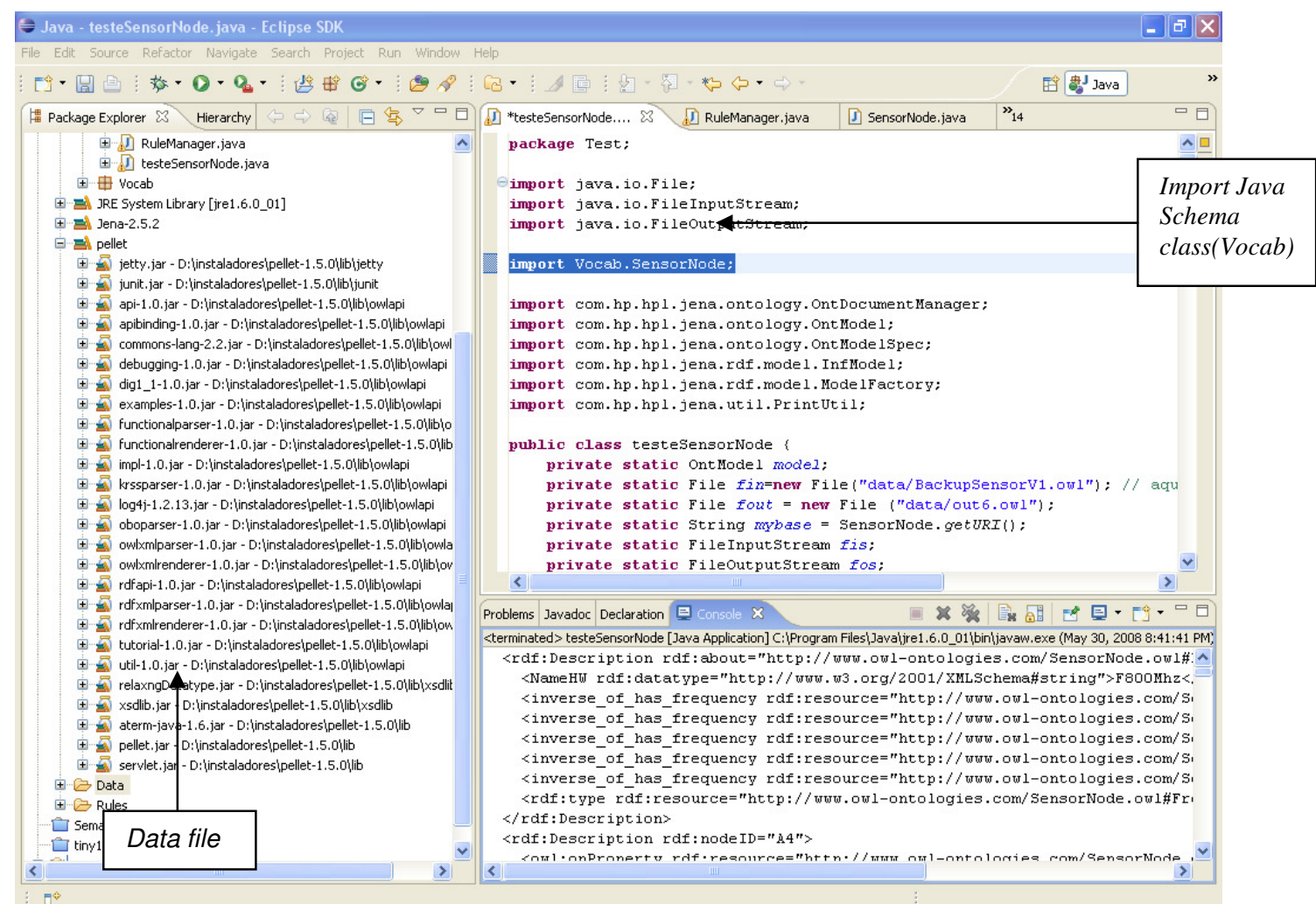

Figura 40 Janela de Eclipse

\section{- Inferência semântica}

Para realizar a inferência semântica das ontologias em Java são realizados dois passos, no primeiro mostra-se como carregar uma ontologia e no segundo a realização da inferência.

No Quadro 3, apresenta-se parte de código em Java para carregar uma ontologia utilizando a biblioteca Jena. O Jena disponibiliza várias formas de carregar uma ontologia (a partir de um arquivo, de um endereço na Web ou de um stream de dados). Para carregar uma ontologia, basta instanciar um objeto OntModel e requisitar ao mesmo que recupere a ontologia utilizando o método read do mesmo. 
Na primeira parte do código são definidas várias variáveis, é definida uma variável (fin) que contém o endereço da pasta com as ontologias; é definida uma variável(mybase), que contém o NameSpace da ontologia; é defina a variável (PAD_ONT_ROOT_URL ) que contém o URL da ontologia; é definida a variável (ontMgt) para criar o gerenciador (manager) para a manipulação das ontologias; no procedimento loadOntology() é criada uma ontologia, e finalmente carrega a ontologia.

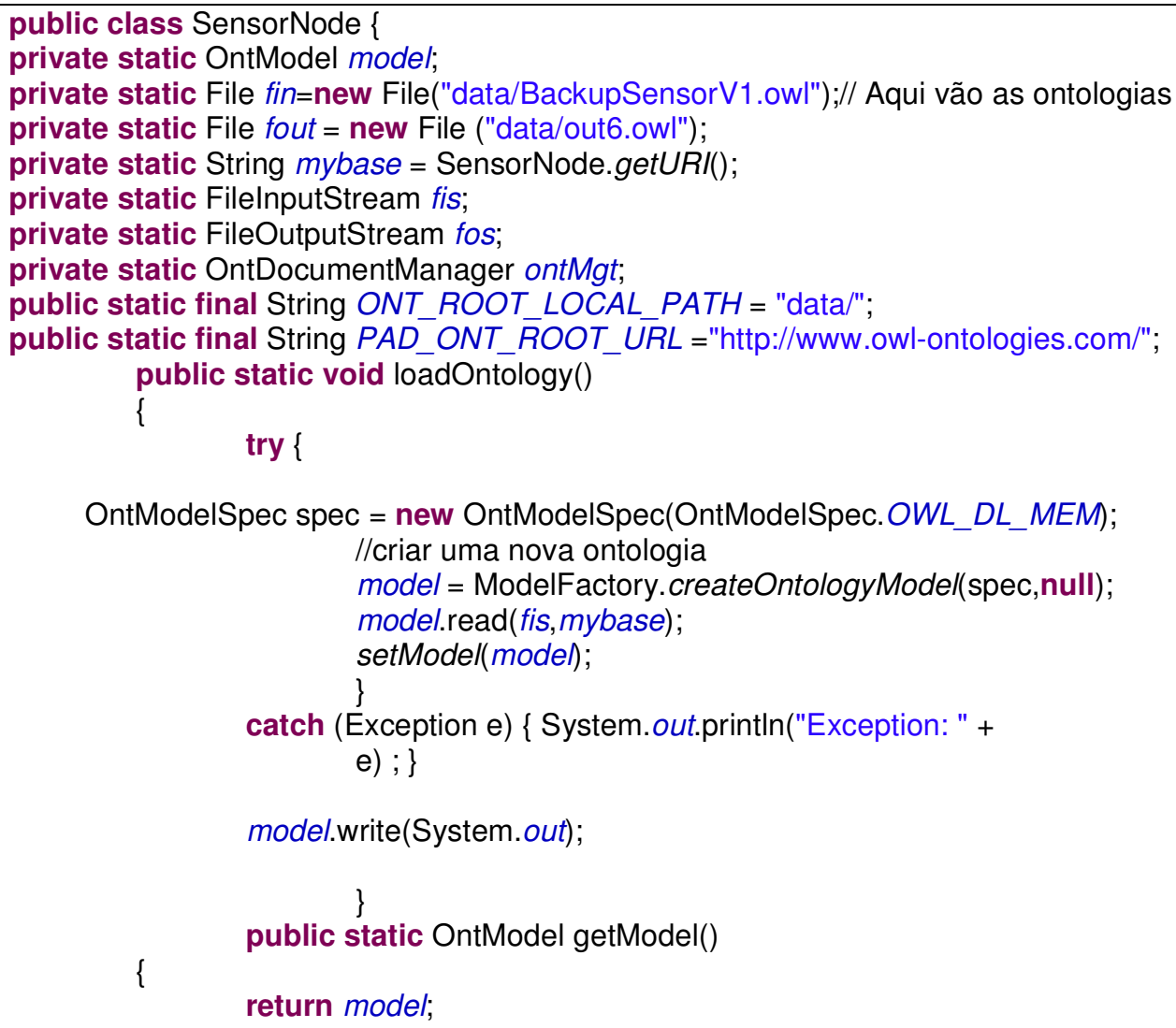

Quadro 3 Parte de código em Java para carregar uma ontologia

No Quadro 4, apresenta-se parte de código em Java para realizar inferência utilizando a maquina de inferência Pellet (HP 2006). Na primeira parte do código é criada a máquina de inferência e carregada a ontologia, verifica-se se a ontologia é consistente usando os serviços de inferência DL, tal como, reasoned.getK $B($ ).isConsistent(), se fora consistente continua a realizar a inferência fazendo uso de reasoner.getKB().realize() e reasoned.getKB().classify(). No Caso 
contrário se mostra uma mensagem de inconsistência. Uma vez carregada a ontologia e realizada a inferência, pode-se prosseguir a fazer consultas usando SPARQL e regras usando Jena Rules.

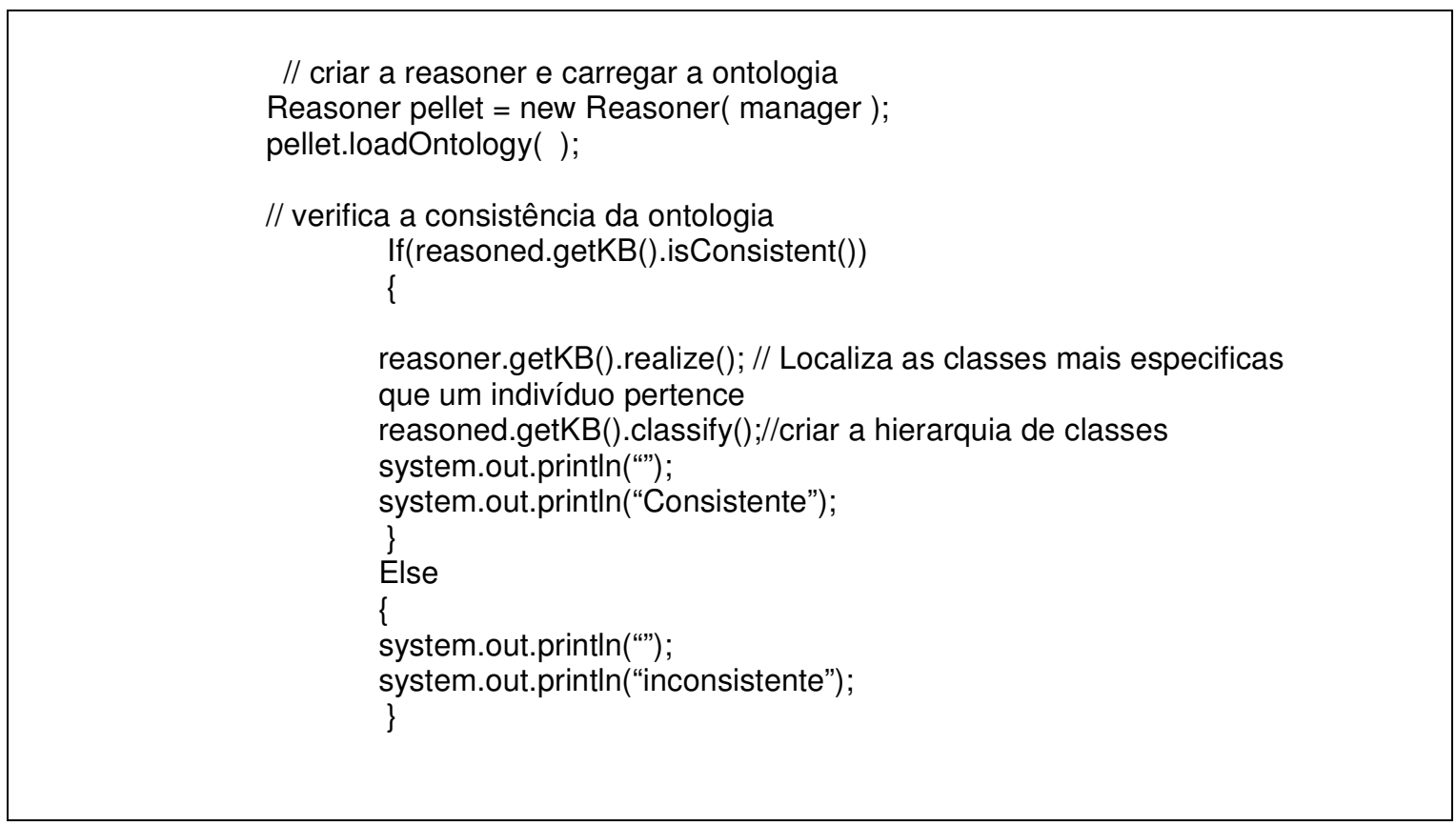

Quadro 4 Parte do código para realizar inferência

A arquitetura esboçada na seção anterior foi parcialmente implementada através da utilização da API Protégé-OWL, da linguagem de consulta SPARQL e da ferramenta Pellet, para realização de inferência sobre a base de conhecimentos representada em OWL-DL e a IDE Eclipse foi usada como apoio às atividades de implementação.

A prioridade para implementação dos componentes da arquitetura foi definida principalmente em razão da necessidade de avaliação da proposta. Maiores detalhes sobre código e implementação podem ser encontrados no CD adjunto à dissertação. 


\section{B Anexo B - TmoteSky}

O TmoteSky foi projetado e desenvolvido pela Universidade de Berkeley e é distribuído pela Moteiv Corporations, que foi fundada pelos estudantes e doutores pesquisadores da Universidade de Califórnia em Berkeley em novembro do 2003 (MOTEIV, 2007). Estes dispositivos foram projetados com dois objetivos principais: alto grau de tolerância a falhas e fácil desenvolvimento. Utiliza um sistema de rádio conhecido como IEEE 802.15.4 ou popularmente como ZigBee, que trabalha na faixa de freqüência de $2.4 \mathrm{GHz}$ ISM e oferece taxas de transmissão na ordem de $250 \mathrm{Kbps}$, com um alcance rádio de 125 metros aproximadamente. Além disso, inclui um Analog to Digital Converter (ADC) de 12 bits e Digital to Analog Converter (DAC), timer, I2C, SPI, um protocolo Universal Asynchronous Receiver-Transmitter (UART) e um controlador de Direct Memory Access (DMA), como se mostra na Figura 41 e a Figura 42.

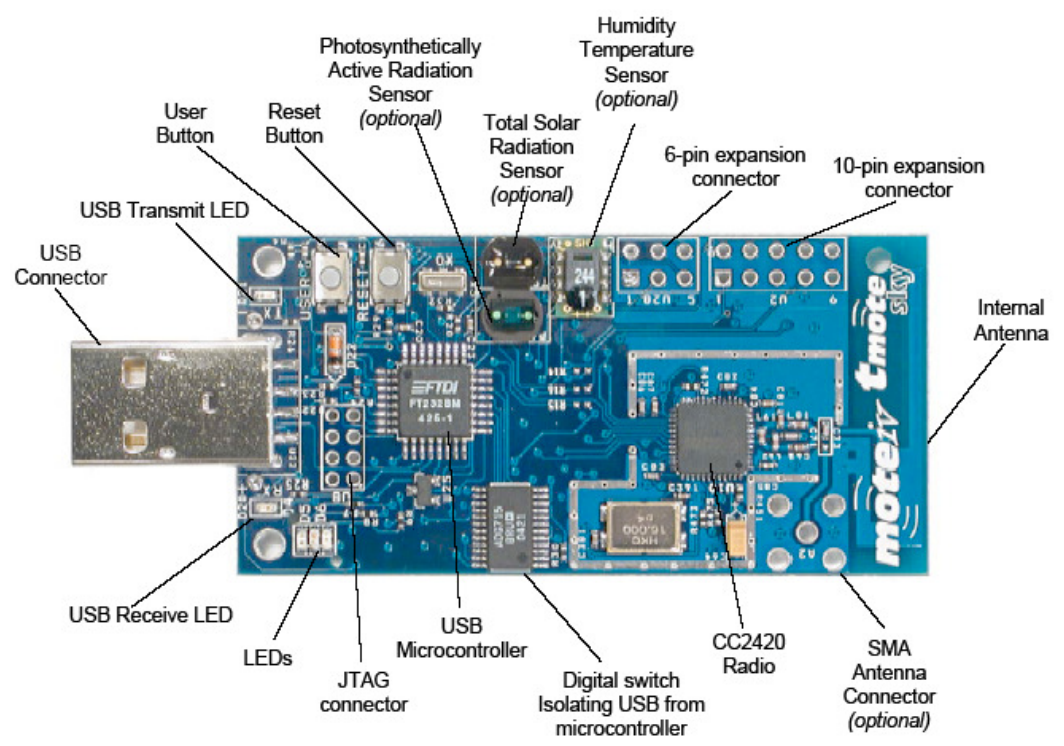

Figura 41 Vista frontal do TmoteSky (MOTEIV, 2007) 


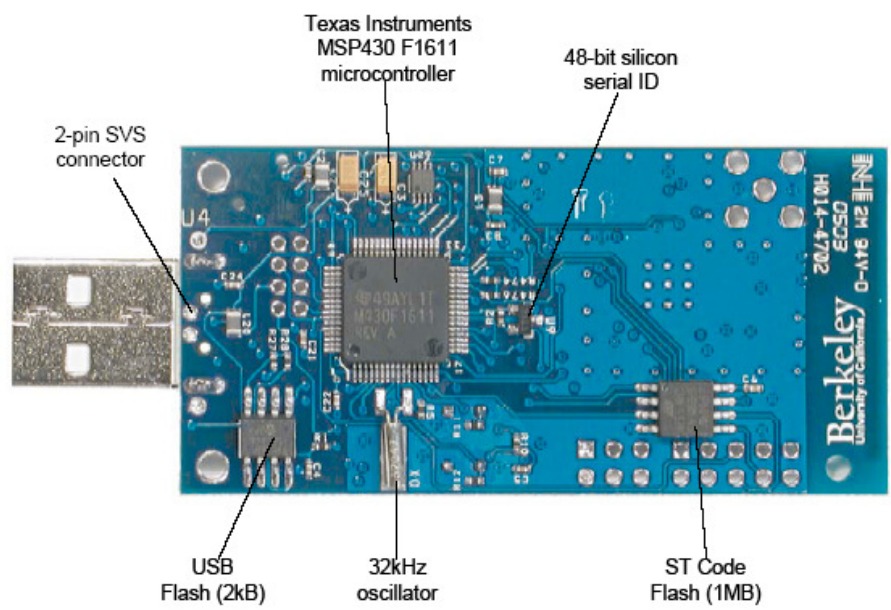

Figura 42 Vista da face inferior do TmoteSky (MOTEIV, 2007)

Os dispositivos utilizam o chip CC2420 desenvolvido pela Chipcon. Este sistema de rádio trabalha com a modulação Quadrature Phase-Shift Keying (QPSK) por meio de DSSS. Outra evolução desta plataforma foi a utilização da interface Universal Serial Bus (USB), que facilita a programação dos dispositivos e a troca de informações com o ponto de acesso.

Na Tabela 4 são apresentadas as características dos nós de sensores como: tipo de processador, memória, rádio, Sistema Operacional das diferentes plataformas de hardware existentes para RSSF. Como se observa na tabela existe na atualidade uma grande quantidade de plataformas para as redes de sensores. Por exemplo, micaZ, Telos, e iMote2, todos eles compartilham o mesmo sistema de rádio CC2420, enquanto Telos, WISAN, eyesIFX, ScartterWeb, usam o microcontrolador MSP430. A família Mica utiliza o microcontrolador Atmega128L.

Tabela 4 Plataformas típicas e componentes de Hardware das RSSF

\begin{tabular}{llll}
\hline Plataforma & Micro controlador & Freqüência & Rádio \\
\hline eyesIFX & TI MSP430 & $2.4 \mathrm{Ghz}$ & TDA5250 \\
ScatterWeb & TI MSP430 & $868 \mathrm{MHz}$ & CC1020 \\
telosB & TI MSP430 & $2.4 \mathrm{Ghz}$ & CC2420 \\
WISAN & TI MSP430 & $2.4 \mathrm{Ghz}$ & CC2420 \\
iMote2 & ARM Core & $2.4 \mathrm{Ghz}$ & CC2420 \\
\hline
\end{tabular}




\begin{tabular}{llll}
\hline micaZ & Atmega128 & $2.4 \mathrm{Ghz}$ & CC2420 \\
mica2 & Atmega128 & $433,868,900 \mathrm{Mhz}$ & CC1000 \\
mica2dot & Atmega128 & $433,868,900 \mathrm{Mhz}$ & CC1000 \\
BTnode & Atmega128 & $433,868,900 \mathrm{Mhz}$ & ZV4002, \\
CC1000 \\
evb13192 & HCS08 & $2.4 \mathrm{Ghz}$ & MC13192 \\
criquet & Atmega128 & $433 \mathrm{Mhz}$ & CC1000 \\
TmoteSky & TI MSP430 & $2.4 \mathrm{Ghz}$ & CC2420 \\
nymph & Atmega128 & $433,868,900 \mathrm{Mhz}$ & $\mathrm{CC} 1000$ \\
XYZ mote & ARM7TDMI & $1.8 \mathrm{MHz}$ & CC2420 \\
TinyNode & MSP430 & $868 \mathrm{MHz}$ ISM & XE1205 \\
mote2 & Intel PXA271 & $2.4 \mathrm{Ghz}$ & CC2420 \\
Schockfish & TI MSP430 & $868 \mathrm{MHz}$ & Xemics XE \\
\hline
\end{tabular}

\section{B.1 Sistema Operacional TinyOS 1.x}

TinyOS 1.x, é um dos primeiros Sistemas Operacionais específicos para as redes sensores, e foi desenvolvido pela Universidade de Berkeley com a colaboração da Intel no início desta década. Em pouco tempo passou a ser o sistema mais popular para RSSF, sendo um software open source disponível em http://www.tinyos.net.

Muitas características do TinyOS $1 . x$ se devem ao fato de ter sido desenvolvido em NesC (LEVIS, et al. 2003), derivado do C ANSI ${ }^{11}$. NesC é uma extensão da linguagem C padrão, adaptada para as necessidades especiais das RSSF. Uma das características principais deste modelo de programação é o uso de componentes. Neste modelo, a funcionalidade da tradicional camada de abstração é quebrada em pequenos blocos que interagem com outros blocos via interface.

Dentre os motivos pelos quais o TinyOS 1.x foi desenvolvido, o principal é a utilização de recursos limitados. Os nós de sensores têm recursos físicos limitados devido ao pequeno tamanho, baixo custo e baixo consumo de energia. $\mathrm{Na}$

\footnotetext{
${ }_{11}$ ANSI, Linguagem de programação criada na década de setenta por Dennis Ritchie e Ken Thompson
} 
atualidade, os nós contêm um processamento de 1 Millions of Instructions Per Second (MIPS) e 10 Kbytes para armazenamento. Em segundo lugar vem a concorrência; em uma aplicação típica de RSSF, um nó é responsável pela amostragem de seu ambiente através dos sensores. Esses sensores são manipulados através dos atuadores, executando processamento de dados, distribuindo dados para outros nós e participando em processos distribuídos, tais como agregação estática. Muitos destes eventos, como por exemplo, a gerência de rádio, requer respostas em tempo real. Isto requer uma abordagem ao gerenciamento de um modelo de concorrência que reduz erros potenciais.

\section{B.2 Aplicativos para visualização dos dados}

Para a visualização dos dados inicialmente utilizamos o Cygwin $^{12}$, para executar o TinyOs. O tratamento dos dados foi feito pela aplicação em Java Listen que escuta os dados que estão chegando pelo aplicativo instalado nos nós sensores. A Figura 43 mostra uma janela exemplo do Listen.

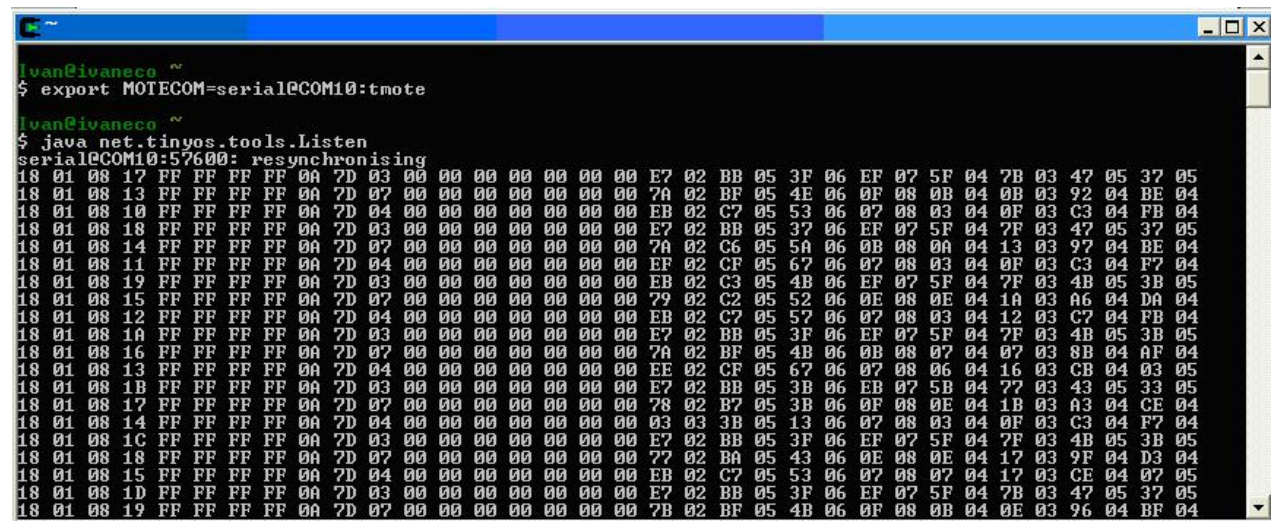

Figura 43 Aplicativo Listen

Existem outras ferramentas de visualização dos dados, por exemplo, Microsoft Excel (CHEONG et al., 2005), LabView (FLORIDO, 2008), entre outras.

${ }^{12}$ Cygwin, biblioteca que implementa a API de chamadas de sistemas POSIX em termos de chamadas de sistemas Win32. 


\section{Anexo C - Instalações}

\section{C.1 Instalação do MSRsense}

O procedimento abaixo descreve como foi feita a instalação da ferramenta em nosso laboratório.

\section{Ferramentas}

- PC, Pentium 4 2.6GHz, 512M memory, 40G+ hard drive,

- Windows Vista ou Windows XP Professional SP2

- Excel 2003 Professional

- Visual Studio 2005 Professional, which also contains:

- Visual Studio 2005 Tools for Office 2003 (a.k.a. VSTO 2005)

- SQL Server Management Studio Express

- TinyOS-1.1.x. (optional)

- Nó sensor TmoteSky

- msrsense-1.0.msi, download da ultima versão em: http://research.microsoft.com/nec/msrsense

\section{O diretório raiz para o código-fonte é:}

c:Program Files\Microsoft Research \MSRSense-1.x

\section{A instalação do MSRSense inclui executáveis pré-construído em .NET 2.0.}

Os executáveis mSEE, mSIC, MoteForwarder estão localizados em : C:IProgram Files\Microsoft ResearchlMSRSense-v1.0lbin.

\section{- mSEE}

Localizado em:

CIProgramFiles\MicrosoftResearch $\backslash M S R S e n s e-1.0 \backslash$ miuSEE $\backslash$ microserver. Utilizando o visual studio 2005, carrega microserver.s/n, para gerar dois arquivos e um executável C:IProgramFiles\MicrosoftResearch \MSRSense-v1.0lbin: Microsoft.NEComp.Microserver.Library.dll 
Microsoft.NEComp.Microserver.dll

miusee.exe

\section{- MoteForwarder}

Solução para converter a conexão (USB) em soquete TCP / IP, para usar o fluxo de dados através de uma interface mais amigável.

Localizado em:

C:IProgram Files/Microsoft Research\MSRSense-v1.0lutillMoteForwarder.

Utilizando o Visual Studio, carrega MoteForwarder.sIn para gerar um executável:

C:IProgramFiles \MicrosoftResearch $\backslash M S R S e n s e-v 1.0 l b i n \backslash$ MoteForwarder.exe.

\section{- $m S I C$}

Localizado em: C:IProgram FilesIMicrosoft ResearchIMSRSense-v1.0lutillmiuSIC.

Utilizando Visual Studio, carrega miuSIC.sIn para gerar o seguinte executável:

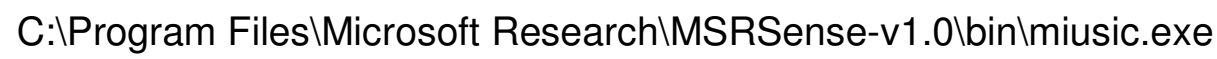

\section{- SF Recorder and Player}

Localizado em: C:IProgramFiles\MicrosoftResearchIMSRSense-1.0lutillSFRecorder. gera dois executáveis:

C:Program Files\Microsoft Research $\backslash M S R S e n s e-v 1.01 b i n \backslash$ Player.exe

C:IProgram Files\Microsoft ResearchlMSRSense-v1.0lbin $\backslash$ Recorder.exe

\section{- Excel Streaming Interface}

Localizado em: C:IProgram Files\Microsoft Research\MSRSense-v1.0Isenscel.

Utilizando Visual Studio, carrega Senscel.sIn para gerar os seguintes arquivos:

C:IProgram Files\Microsoft Research $\backslash M S R S e n s e-v 1.0 \backslash b i n \backslash$

Senscel.CommonLibrary.dll

Senscel.DataAccessLibrary.dll

Senscel.PacketConnectorLibrary.dll

Senscel.dll.config

Senscel.dll

Senscel.xls 


\section{C.2 Instalação do TinyDB}

O procedimento abaixo descreve como foi feita a instalação do tinyDB, primeiramente foi instalado em cada nó sensor a aplicação TinyDBApp.nc, disponível em .../opt/tinyos-1.x/apps/TinyDBApp. Esta aplicação é responsável para gerenciar a coleta das informações especificadas nas consultas SQLs e enviar para o nó sorvedouro, a instrução se mostra no Quadro 5.

$\$ c d$ opt/tinyos-1.x/apps/TinyDBApp/

make mica install, $<01>$

Quadro 5 Instrução para instalar TinyDB

Para trabalhar com o aplicativo TinyDBMain que é responsável por enviar as $S Q L s$ para a RSSF e receber as informações dos sensores. Primeiro tem-se que construir as classes java - para fazer isso, se necessita assegurar que os pacotes JLex.jar, cup.jar, e plot.jar estão em seu CLASSPATH; estes pacotes encontra-se disponível em: tools/java/jars/. Em seguida fazer o make como se mostra no Quadro 6.

\$ cd /opt/tinyos-1.x/tools/java/net/tinyos/tinydb make

Quadro 6 Instrução para a instalação do TinyDBMain

Finalmente para mostrar a tela do Graphical User Interface (GUI). O Quadro 7 mostra a instrução que deve ser executada a partir do diretório. ..../tool/java.

cd ........

... /opt/tinyos-1.x/tools/java

java net.tinyos.tinydb.TinyDBMain 